\title{
Reduction Procedures in Fraction Arithmetic
}

by

\author{
Shawn Tan
}

A thesis submitted to the Faculty of Graduate and Postdoctoral Affairs in partial fulfillment of the requirements for the degree of

Doctor of Philosophy

In

Cognitive Science

Carleton University

Ottawa, Ontario

(C) 2020

Shawn Tan 


\begin{abstract}
In elementary school, students learn about fraction concepts and procedures. Concepts are knowledge about fraction facts and principles, for example, that two different fractions (i.e., $4 / 8$ and 5/10) can have the same value. Procedures are algorithms that, when applied correctly, allow students to produce a correct solution (e.g., addition, subtraction, multiplication, and division). Knowledge of fraction procedures and understanding how those procedures apply is central to mathematical knowledge that students acquire in school and is used frequently in everyday life. The goal of the present research was to explore adults' use of a fraction procedure called reduction, a procedure that is taught to students but is sometimes an optional step in fraction arithmetic. Fraction reduction involves dividing the numerator and denominator of a fraction by a common factor. However, there is no existing research on fraction reduction, despite considerable interest in how students learn and apply fraction procedures.

In three studies, I assessed the factors that influence adults' use of fraction reduction. I hypothesized that characteristics of the solvers and of the problems would both contribute to people's use of reduction in fraction arithmetic. In Study 1, 720 participants were assessed on their conceptual and procedural understanding of fractions and profiled as either conceptually- or procedurally-stronger solvers. I found that solvers' strategy selection was related to their understanding of fraction concepts and procedures, the required operation, and whether fractions within a problem shared a common denominator (i.e., denominator-relation). In a subsequent study with two cohorts, a smaller group of 59 participants from the larger sample solved fraction arithmetic problems and provided written protocols. Results from the second study suggest that a combination of denominator-relation, and operation predicted use of reduction during fraction
\end{abstract} arithmetic. However, when solvers multiplied fractions, conceptually-stronger solvers were more 
likely to reduce fractions than procedurally-stronger solvers. These findings are discussed in relation to the potential impact of fraction concepts and procedures on selection of the reduction procedure. 


\section{Acknowledgements}

Firstly, I would like to thank my supervisor Dr. Jo-Anne LeFevre for her most thorough supervision and editing of my manuscript, her advice and support, as well as her understanding as I underwent an eye-operation during the course of completing my dissertation. Secondly, I would like to thank my dissertation committee members Drs. Kasia Muldner and Craig LethSteensen for providing helpful comments in improving my manuscript and for clarifying my thought process. Finally, I would like to thank members of the Math Lab at Carleton University for helping me edit and prepare my presentations and for their understanding and support during the dissertation process. 


\section{Table of Contents}

Abstract

CHAPTER 1: INTRODUCTION

Conceptual and Procedural Knowledge of Fractions 2

Overview of the Dissertation 3

The Present Research 5

Outline of the Dissertation $\quad 8$

CHAPTER 2: BACKGROUND AND LITERATURE REVIEW 13

Background: Fractions and Fraction Arithmetic 13

Fraction Addition and Subtraction $\quad 13$

Fraction Multiplication 14

Other Fraction Procedures $\quad 15$

Conversion of fractions to mixed numbers. 15

Conversion of mixed numbers to improper fractions. 16

Reduction. 16

$\begin{array}{ll}\text { Cross-reduction. } & 16\end{array}$

$\begin{array}{ll}\text { Literature Review } & 18\end{array}$

What makes Fraction Arithmetic Difficult? 18

Conceptual Knowledge, Procedural Knowledge, and Reduction 20

The Role of Reduction $\quad 23$

Current Theories Relevant to Fraction Arithmetic 25

Errors generated by middle school solvers. $\quad 27$

A model of fraction arithmetic (FARRA). 28

The identical elements model of whole-number arithmetic 34

CHAPTER 3: STUDY $1 \quad 36$

$\begin{array}{ll}\text { Method } & 39\end{array}$

Participants $\quad 39$

$\begin{array}{ll}\text { Materials } & 39\end{array}$

Mathematics Background and Interests Questionnaire (MBIQ) 40

Calculation Fluency Test (CFT). 
Basic Multiplication Test (BMT).

Fraction Knowledge Assessment (FKA) 42

$\begin{array}{ll}\text { Conceptual items } & 42\end{array}$

$\begin{array}{ll}\text { Procedural items } & 44\end{array}$

Fraction arithmetic problems $\quad 45$

Results $\quad 46$

$\begin{array}{ll}\text { Exclusion criteria } & 46\end{array}$

$\begin{array}{ll}\text { Descriptives. } & 47\end{array}$

$\begin{array}{ll}\text { Analysis } & 48\end{array}$

Fraction Knowledge Profiles $\quad 49$

Cluster analysis of standardized residualized scores 49

Basic arithmetic test scores for fraction knowledge profiles 55

BMT and CFT scores $\quad 55$

MBIQ scores $\quad 55$

$\begin{array}{ll}\text { Discussion } & 58\end{array}$

What Fraction Knowledge Profiles were Observed for Adults? 58

Characteristics of Each Fraction Knowledge Group 58

CHAPTER 4: FRACTION ARITHMETIC PROBLEMS (FARRA) 60

$\begin{array}{ll}\text { Results } & 64\end{array}$

$\begin{array}{ll}\text { Accuracy } & 64\end{array}$

Most Frequent Responses $\quad 66$

$\begin{array}{ll}\text { Correct strategies } & 67\end{array}$

$\begin{array}{ll}\text { Conversion to decimals } & 67\end{array}$

$\begin{array}{ll}\text { Reduction } & 69\end{array}$

Incorrect strategies

Independent whole number strategy $\quad 72$

$\begin{array}{ll}\text { Wrong fraction operation strategy } & 74\end{array}$

$\begin{array}{ll}\text { Wrong inversion strategy } & 77\end{array}$

$\begin{array}{ll}\text { No response } & 79\end{array}$

$\begin{array}{lr}\text { Discussion } & \mathbf{8 0}\end{array}$ 
What are the Factors that Influence Adults to Select Correct Strategies

What Incorrect Strategies do Adults Apply when they Generate Systematic Errors? 81

What are the Factors that Influence Adults' Selection of Incorrect Strategies? 83

Fraction Arithmetic and Fraction Reduction $\quad 85$

$\begin{array}{ll}\text { Limitations } & 86\end{array}$

The Solution: A Follow-up Study $\quad 87$

CHAPTER 5: STUDY 2 (PART 1: WRITTEN PROTOCOLS) 88

$\begin{array}{ll}\text { Overview of Study } 2 & 88\end{array}$

$\begin{array}{ll}\text { Hypotheses } & 90\end{array}$

$\begin{array}{ll}\text { Method } & 90\end{array}$

$\begin{array}{ll}\text { Participants } & 90\end{array}$

$\begin{array}{ll}\text { Materials } & 91\end{array}$

$\begin{array}{ll}\text { Procedure } & 93\end{array}$

$\begin{array}{ll}\text { Results } & 93\end{array}$

Descriptives and Group Comparisons $\quad 93$

Accuracy on Experimental Trials 95

Analysis of Written Protocols $\quad 97$

$\begin{array}{ll}\text { Reduction Use } & 98\end{array}$

Reduction-related Strategies $\quad 99$

Nonreduction 100

Reduction-before-solution 100

Reduction-after-solution $\quad 100$

$\begin{array}{ll}\text { Summary } & 102\end{array}$

$\begin{array}{ll}\text { Incorrect Responses } & 102\end{array}$

Addition and subtraction $\quad 103$

$\begin{array}{ll}\text { Computation errors } & 104\end{array}$

$\begin{array}{ll}\text { Number substitution errors } & 104\end{array}$

$\begin{array}{ll}\text { Operation substitution errors } & 105\end{array}$

$\begin{array}{ll}\text { Combination errors } & 105\end{array}$

$\begin{array}{ll}\text { Other errors } & 106\end{array}$ 
$\begin{array}{ll}\text { Summary for addition and subtraction } & 106\end{array}$

Multiplication 106

$\begin{array}{ll}\text { Strategy selection errors } & 106\end{array}$

$\begin{array}{ll}\text { Operation-substitution errors } & 107\end{array}$

$\begin{array}{ll}\text { Summary for multiplication errors } & 107\end{array}$

$\begin{array}{ll}\text { Division } & 108\end{array}$

$\begin{array}{ll}\text { Strategy selection errors } & 108\end{array}$

$\begin{array}{ll}\text { Number substitution errors } & 108\end{array}$

$\begin{array}{ll}\text { Computation errors } & 108\end{array}$

$\begin{array}{ll}\text { Unique errors } & 109\end{array}$

Summary for division $\quad 109$

$\begin{array}{ll}\text { Discussion } & 109\end{array}$

The Source of Errors in Fraction Arithmetic $\quad 111$

Fraction Multiplication and Reduction $\quad 113$

CHAPTER 6: LATENCIES AND EYE TRAJECTORIES 115

Are Reducible Fraction Multiplication Problems Solved More Quickly and Accurately Than Non-Reducible Problems? 115

Where Do Solvers Focus Their Attention When They Multiply Fractions? 117

$\begin{array}{ll}\text { Hypotheses } & 119\end{array}$

$\begin{array}{lr}\text { Method } & 120\end{array}$

$\begin{array}{ll}\text { Participants } & 120\end{array}$

$\begin{array}{ll}\text { Materials } & 120\end{array}$

$\begin{array}{ll}\text { Fraction arithmetic problems } & 120\end{array}$

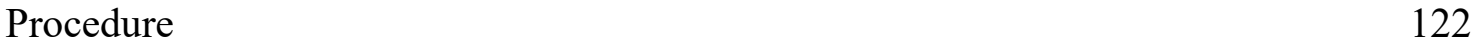

Non eye-tracking cohort $\quad 122$

Eye-tracking cohort 123

$\begin{array}{ll}\text { Results } & 125\end{array}$

$\begin{array}{ll}\text { Problem Solving Performance } & 126\end{array}$

$\begin{array}{ll}\text { Accuracy } & 126\end{array}$

$\begin{array}{ll}\text { Use of reduction. } & 127\end{array}$ 
$\begin{array}{ll}\text { Latencies. } & 129\end{array}$

Fixation Dyads 132

$\begin{array}{ll}\text { Discussion } & 136\end{array}$

Are Reducible Fraction Multiplication Problems Solved More Quickly and Accurately Than Non-Reducible Problems? 136

Where Do Solvers Focus Their Attention When They Multiply Fractions? 137

$\begin{array}{ll}\text { Limitations } & 138\end{array}$

$\begin{array}{ll}\text { Summary } & 139\end{array}$

CHAPTER 7: GENERAL DISCUSSION AND IMPLICATIONS 141

Fraction Knowledge Profiles $\quad 141$

Differences in Strategy Selection across Solver Profiles 142

Differences in Use of Reduction Across Studies $\quad 145$

A Framework of Reduction Use in Fraction Multiplication $\quad 152$

Reduction after multiplication 153

Reduction before multiplication 154

Reduction Involves Both Conceptual and Procedural Knowledge 154

Implications for the FARRA model 156

$\begin{array}{ll}\text { Limitations } & 159\end{array}$

Instruction Confounds 159

Problem Confounds 160

Participant Confounds 160

Multiple Analyses 161

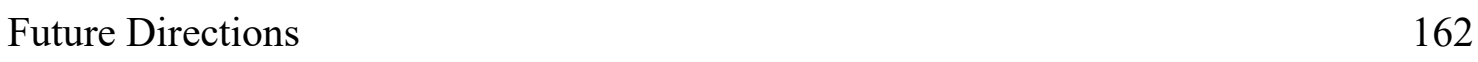

$\begin{array}{ll}\text { Conclusions } & 163\end{array}$

CHAPTER 8: REFERENCES 165 


\section{List of Figures}

Figure 2.1. Fraction Addition

Figure 2.2. Fraction Subtraction

14

Figure 2.3. Fraction Multiplication

14

Figure 2.4. Fraction Division

Figure 2.5. Reduction

17

Figure 2.6. Cross-Reduction

Figure 3.1: Scatterplot for participants standardized residualized conceptual and procedural scores obtained by members of Group 1: the procedurally-stronger group $(n=346)$ and Group 2: the conceptually-stronger group $(n=374)$.

Figure 3.2: Average means for standardized residualized conceptual and procedural scores obtained by members of Group 1: the procedurally-stronger group ( $n=346)$ and Group 2: the conceptually-stronger group $(n=374)$. Error bars represent the standard error of the mean.

Figure 3.3: Mean conceptual and procedural scores for the procedurally-stronger group $(n=346)$ and conceptually-stronger group $(n=374)$. Error bars are 95\% CI calculated according to Jarmasz \& Hollands, (2009).

Figure 4.1. Percentage scores obtained by procedurally- and conceptuallystronger solvers by operation (addition, subtraction, multiplication and division) and whether each problem had the same or different denominators.

Figure 4.2. Average percentage of correct trials on which participants converted 68 fractions into decimals by operation and denominator relationship. 
Figure 4.3. Average frequency of selections (in \%) for correct reduced solutions

by operation and group.

Figure 4.4. Average frequency of use of the independent whole number strategy by group.

Figure 4.5. Average frequency of selections (in \%) for the wrong fraction operation strategy.

Figure 4.6. Average frequency of selections (in \%) for the wrong fraction inversion strategy.

Figure 4.7. Average frequency of blank answers (in \%).

Figure 5.1: Participant characteristics across Studies 1 and 2; and across parts and cohorts for Study 2.

Figure 5.2: Percentage scores obtained by procedurally-and conceptuallystronger solvers by operation and denominator relationship.

Figure 5.3: Average frequency of correct reduction use obtained by procedurallyand conceptually-stronger solvers by operation and denominator relationship.

Figure 5.4. Percentage use of non-reduced and reduced solutions in written protocols. Error bars are 95\% CI calculated according to Jumasz \& Holland (2009).

Figure 6.1. Pairs of fixations on fraction components that correspond to identified algorithmic steps for fraction multiplication.

Figure 6.2. Outline of the timeframe of a typical problem in the non eye-tracking cohort of Study 2 
Figure 6.3. Outline of the timeframe of a typical problem in the eye-tracking cohort of Study 2

Figure 6.4. Dimensions of interest regions centered on problem elements for a typical problem in the eye-tracking cohort

Figure 6.5. Percentage accuracy scores obtained by solver group and problem type.

Figure 6.6: Means of median latencies (in ms) by solver group and problem type.

Figure 6.7: Means of median latencies (in ms) for reducers and non-reducers by problem type.

Figure 6.8: Average number of fixation dyads by dyad type (vertical vs. diagonal vs. horizontal) and solver group (procedurally-and conceptually-stronger solvers 


\section{List of Tables}

Table 1.1. Overview of questions that were addressed for each experiment and the methods that were used

Table 2.1: Firing of correct strategy and execution production rules for the multiplication problem $1 / 4 \times 3 / 4$

Table 3.1. Measures.

Table 3.2. Principles tested in the conceptual FKA and examples

Table 3.3. Examples of procedural items from the Fraction Knowledge Assessment (FKA).

Table 3.4: Fraction arithmetic problems presented to participants in Study 1

Table 3.5: The number of participants $(N)$ that completed each assessment, average time (in min) and standard deviation taken to complete each assessment. Each section of the CFT and BMT had a fixed time limit of $1 \mathrm{~min}(60 \mathrm{~s})$.

Table 3.6. Descriptive Statistics for each Mathematical Measure

Table 3.7: $c(g)$ values for $k$-means cluster solutions using standardized residual scores for conceptual and procedural knowledge.

Table 3.8: Means and standard deviations of procedurally- and conceptuallystronger solvers on the total CFT, BMT (small), BMT (large) and fraction arithmetic problems (\% correct).

Table 4.1. Percent use of fraction arithmetic strategies in $8^{\text {th }}$ grade students 
Table 5.1. Fraction arithmetic problems in Part 1 of Study 2

Table 5.2: Means and standard deviations of conceptually-stronger and procedurally-stronger solvers. Conceptual and procedural scores are the standardized residuals.

Table 5.3: Percentage (of total trials) on which participants made specific types of errors grouped by operation and denominator relationship

Table 6.1. Pairs of fixations upon fraction components that correspond to identified algorithmic steps for fraction multiplication problems.

Table 6.2. Unit-reducible, cross-reducible, and non-reducible multiplications problems used in the second part of Study 2.

Table 6.3. Characteristics of participants in the eye-tracking and non-eye-tracking cohort. Median age in years.

Table 6.4. Means (and standard errors) of the number of fixation dyads by solver group (procedurally-stronger vs. conceptually strong solvers), problem type (reducible vs. cross-reducible vs. non-reducible) and fixation dyad type (vertical vs. diagonal vs. horizontal).

Table 6.5. Means (and standard errors) of the number of fixation dyads by solver group (procedurally-stronger vs. conceptually strong solvers), problem type (reducible vs. cross-reducible vs. non-reducible) and fixation dyad type (vertical vs. diagonal vs. horizontal).

Table 7.1. Comparison of effects of factors on correct reduction-use across studies for multiplication and division problems presented across studies. 
Table 7.2. Two possible elaborations of the algorithm for reducible multiplication

problems: The reduction after multiplication versus reduction before multiplication.

Table 7.3. Strategy rules for implementing reduction in FARRA 


\section{List of Appendices}

APPENDIX A: Rules implemented in FARRA 173

APPENDIX B: Mathematics Background and Interests Questionnaire (MBIQ) 175

APPENDIX C: Fraction Knowledge Assessment(FKA) 178

APPENDIX D: Descriptive Statistics for Participants in Study $1 \quad \mathbf{1 8 3}$

APPENDIX E: Descriptives for MBIQ $\quad 184$

APPENDIX F: Inferential Statistics for MBIQ $\quad \mathbf{1 8 5}$

$\begin{array}{ll}\text { APPENDIX G: Responses for FARRA problems in Study } 1 & \mathbf{1 8 7}\end{array}$

APPENDIX H: Responses for Fraction arithmetic problems in Study 2- Part I 195 


\section{CHAPTER 1: INTRODUCTION}

Children have difficulty learning and performing tasks that involve fractions. For example, in a national assessment of 20,000 grade 8 students (13 to 14 years old) in the United States, children were required to choose the closest whole number to the sum of 12/13 + 7/8. The response options were "1", “2”, “19”, “21" and "I don't know". Only $27 \%$ of the children gave the correct answer " 2 ", indicating that the majority of children had trouble understanding fractions (Carpenter, Corbitt, Kepner, Lundqvist, \& Reys, 1980). More than 30 years later, Lortie-Forgues, Tian, and Siegler (2015) asked the same question of 48 grade 8 students and similarly reported that only $24 \%$ gave the correct answer. Thus, despite decades of research, children's knowledge of fraction concepts and procedures is weak (Behr, Wachsmuth, \& Post, 1985; Hecht, 1998; Hecht \& Vagi, 2012, Siegler, Fazio, Bailey \& Zhou, 2013; Siegler \& LortieForgues, 2015, Siegler \& Pyke, 2013) and even adults have difficulty understanding and using fractions correctly (Bentley \& Bosse, 2018; Stigler, Givvin, \& Thompson, 2010).

The goal of the present research was to use multiple measures to explore fraction understanding in adults. To achieve this goal, I focused on a particular type of conceptual and procedural knowledge that people use when solving fraction problems, that is, knowledge of how and when to simplify fractions such as $2 / 4$ or $8 / 10$. Reducing fractions to their simplest form taps into fraction knowledge at both the conceptual and procedural level, as I will demonstrate in this dissertation. On the one hand, knowing how and when to use reduction requires knowledge of concepts because solvers have to understand that the fractions $4 / 5$ and $8 / 10$ have the same magnitude even though they are composed of different numbers. Solvers also have to understand that a fraction such as $8 / 10$ can be reduced to a simpler form, whereas the 
fraction $7 / 10$ cannot be reduced. On the other hand, knowing how to apply the reduction application also requires procedural knowledge because solvers need to know how to convert fraction with larger components such as 8/10 into its simplest form, that is, $4 / 5$. There are two general questions that will be addressed in this dissertation. First, I identify how solvers use their conceptual and procedural knowledge of fractions to simplify fraction problems by reducing fractions (i.e., reduction). Second, I address what kinds of problems facilitate the use of reduction.

\section{Conceptual and Procedural Knowledge of Fractions}

Two types of fraction knowledge contribute to people's understanding of fractions (Hallett, Nunes \& Bryant, 2010; Hecht, 1998; Siegler, Fazio, Bailey \& Zhou, 2013). Conceptual knowledge has been broadly defined as knowledge that is "rich in relationships" (Hiebert \& Lefevre, 1986). Conceptual knowledge is not just knowledge of a fact or a principle, but also includes how a fact or a principle is connected to other facts or principles (Crooks \& Alibali, 2014). Educational researchers have identified a wide range of cognitive abilities and processes that affect children's learning of fraction concepts and procedures (Bailey, Hansen, \& Jordan, 2017; Hallett et al, 2010; Hecht, 1998; Hecht \& Vagi, 2012). The set of skills that encompasses conceptual fraction knowledge includes how components of a fraction are related to each other. For example, conceptual knowledge includes the knowledge that, for the fraction $1 / 2$, the numerator 1 and the denominator 2 are related to one another and both numerator and denominator are related to the magnitude of the fraction. Conceptual knowledge also involves the knowledge that $1 / 2$ and 2/4 are related to each other in that both fractions denote the same quantity. Finally, conceptual knowledge includes knowledge of relative magnitude, for example, that 7/9 has a larger value than 5/9 (Siegler et al, 2013). 
In contrast, procedural knowledge involves both the knowledge of the steps in a solution algorithm and the order of those steps to produce a correct solution to a problem (Rittle-Johnson \& Siegler, 1998). For example, adding two fractions such as $1 / 2$ and $2 / 4$ requires problems solvers to first ensure that the two fractions share a common denominator (i.e., by reducing $2 / 4$ to $1 / 2$ or by changing $1 / 2$ to $2 / 4$ ), then adding the numerators of the converted fraction(s) while retaining the denominator of the fractions. (See also the section titled Background: Fractions and fraction arithmetic for more examples of procedures used in fraction arithmetic). Researchers who study solvers' ability to use procedures to solve fraction problems focus on understanding individual differences in fraction knowledge, differences in how fractions are learned and how fraction skills develop, and subsequently how individual knowledge about fractions influences problems solvers' ability to perform efficiently (Bailey et al. ,2017; Hallett et al., 2012; Jordan, Hansen, Fuchs, Siegler, Gersten \& Micklos, 2013).

\section{Overview of the Dissertation}

Understanding fractions is a precursor to developing higher-level skills, for example, in algebra (Booth \& Newton, 2012). Fraction knowledge is also important for solving equations in the maths and sciences and complements understanding of ratios, proportions, and percentages (Hurst \& Cordes, 2016). In the Ontario Curriculum, middle school students are expected to be able to work with both fraction concepts and procedures. By Grade 6, students, are expected to understand that two or more fractions can have equivalent values, compare the magnitudes of two fractions, and to determine whether two fractions have the same magnitudes. By Grade 8, students are expected to be able to add, subtract, multiply and divide fractions (The Ontario Curriculum, 2018 ). 
Given the pervasive difficulty that people have with fractions, there is a need to understand the cognitive processes that people use in this specific area of numerical cognition. The goal of the present thesis is to provide an in-depth analysis of the processes that adults use to solve fractions with a focus on one aspect of fraction knowledge, that is, fraction reduction. I focused on fraction reduction, as described below, because knowledge of this aspect of fraction processing will, I contend, provide insight into both procedural and conceptual aspects of fraction knowledge among adults.

Fraction reduction is a procedure that people use to reduce more complex fractions to simpler fractions by dividing both the numerator and denominator of a fraction by a common factor. For example, the fraction 33/99 can be reduced to it simplest form, $1 / 3$, by dividing both components by 33 . Fraction reduction is a procedure, because it is the algorithmic process of transforming a more complex fraction to a simpler yet equivalent fraction. However, deciding when reduction will be useful can be considered conceptual knowledge, because it involves the knowledge that two fractions can be equivalent and the knowledge of the conditions about when reduction can be applied. There is no specific expectation in the Ontario Curriculum about whether students should be able to solve problems using reduction. Thus, students are not expected to be able to apply reduction in the Ontario schools, but have to rely on their conceptual and procedural fraction knowledge and/or instruction from their teachers to understand when to reduce fractions. Furthermore, there is no specific focus about the types of knowledge that solvers need to be able to use reduction or the conditions under which reduction occurs, even though the reduction procedure has been reported as a strategy that is used when solvers perform fraction arithmetic (Freudenthal, 2002; Olive, 1999). 


\section{The Present Research}

In this dissertation, I will focus on two fraction procedures, reduction and cross-reduction. Reduction itself is a procedure, but when and how to reduce fractions may also involve conceptual knowledge, in the sense that knowledge of reduction entails knowing a specific fact about the relation between two fractions. For example, two fractions such as $2 / 4$ and $1 / 2$ have the same magnitude despite both fractions having different numerators and denominators, whereas fractions such as $2 / 5$ and $3 / 5$ have different magnitudes even though they share a common denominator. One reason to study fraction reduction, therefore, is to understand how conceptual and procedural knowledge work together to support fraction arithmetic. A second reason to study fraction reduction is that it may be useful for generating and testing models of strategy selection and execution in fraction arithmetic. Fraction arithmetic problems can be solved using a sequence of steps that will lead to the correct solution. Reduction procedures are not applicable to every fraction arithmetic problem. However, for more complex problems, such as $7 / 14 \times 3 / 5$, reducing $7 / 14$ to the simpler fraction $1 / 2$ may be beneficial, allowing the correct solution to be obtained more quickly and/or accurately.

Despite the importance of understanding the role of reduction procedures and the apparent advantages that reduction might provide to perform fraction arithmetic, a review of the current literature reveals no research on what solvers need to understand about fractions to perform reduction. Thus, a focus on how reduction can be correctly implemented to solve fraction arithmetic problems, that incorporates both procedural and conceptual fraction knowledge, will be an important contribution to research on the cognitive processes involved in fraction arithmetic.

In this dissertation, I attempted to identify the types of fraction knowledge and 
the characteristics of problems that guide adults to reduce fractions. Knowledge of how and when solvers reduce fractions has important implications for research on fraction arithmetic for three reasons. Firstly, reduction requires the conceptual understanding that two fractions can have identical values and understanding of the conditions that allow reduction to be applied. Secondly, reduction requires knowledge of the procedure to reduce fractions to their simplest form. Thirdly, although reduction is not essential to solve problems, reducing fractions with large numerators and denominators to fractions with simpler components allows solvers to work with and perform basic arithmetic on smaller components. Presumably, therefore, reduction reduces the overall complexity and cognitive load of fraction arithmetic.

Because reduction plays several roles in fraction processing, I contend that it will be a useful forum in which to explore adults' fraction performance. These three reasons link conceptual and procedural knowledge and provide an overview for the questions that will be addressed in this dissertation. The knowledge that two or more fractions can have equal values (i.e., $6 / 9=4 / 6=2 / 3$ ) requires the solver to have a conceptual understanding that two fractions can have the same value. However, understanding the conceptual equivalence of two fractions does not specify how to apply the reduction algorithm to obtain equivalent fractions (e.g., How do you obtain a fraction with the same value as $35 / 45$ ?). The procedural knowledge of how to reduce fractions requires the solver to understand that a numerator-denominator pair with a common factor can be reduced and specifies the steps to perform the procedure. Procedural knowledge of how to reduce fractions requires solvers to first identify a common factor between numerators and denominators of a fraction and second, to reduce the numerator-denominator pair by that factor. In the current literature, there is no research that (1) focuses on reduction, (2) identifies the factors 
that cue solvers to apply reduction, and (3) shows if solvers attempt to apply reduction for every fraction, or only under specific conditions.

This dissertation provides an exploratory investigation into when solvers use reduction. The dissertation is exploratory because there is no previous research about the factors that facilitate people to use reduction when performing arithmetic. Thus, the questions posed in this dissertation about reduction attempt to differentiate between the conditions about when solvers apply the reduction strategy based on previously identified factors that cue solvers to select strategies as they perform fraction arithmetic. The general questions answered in the dissertation are as follows: First, what fraction knowledge profiles are observed for adults? Second, what factors cue adults to select strategies as they perform fraction arithmetic? Third, what are the problem characteristics and solver profiles that facilitate solvers to reduce fractions? Fourth, are reducible fraction multiplication problems solved more accurately and quickly than non-reducible problems? Fifth, where do solvers focus their attention when they multiply fractions?

To address these questions, I profiled problem solvers' knowledge of fraction concepts and procedures according to the cluster analysis technique described by Hallett and his colleagues (Hallett et al., 2010; 2012). I inferred strategies from solvers' responses as they performed fraction arithmetic and assessed whether fraction knowledge as well as previously identified problem characteristics - namely fraction operation and denominator relationship (see Siegler, Thompson \& Schneider, 2011) cued solvers to select strategies. I then assessed whether solvers' profiles and problem characteristics were related to the frequency and appropriate use of fraction reduction. I determined whether application of the fraction reduction algorithm was efficient and effective in assisting solvers generate correct solutions quickly for fraction 
multiplication problems by comparing solvers' accuracy and problem-solving times for reducible and non-reducible problems. I tracked solvers' eye patterns to evaluate the extent to which eye-tracking could be used to provide evidence for the reduction algorithm when solvers multiply fraction. Finally, I provide a discussion of the factors that cue solvers to use reduction, and how findings from my dissertation might inform other areas of research in fraction arithmetic and mathematics.

\section{Outline of the Dissertation}

The outline of the dissertation and the questions are presented in Table 1.1 (page 12). These goals will be studied within the framework of how solvers represent and apply their fraction arithmetic knowledge to solve problems.

In Chapter 2, I provide a review of fractions and the steps used to solve fraction arithmetic problems, review the literature on procedural and conceptual knowledge required to solve fraction arithmetic problems, and describe a computational model that implements the algorithmic steps used to solve fraction arithmetic problems (i.e., FARRA; Braithwaite, Pyke \& Siegler, 2017). FARRA (Fraction Arithmetic Reflects Rules and Associations) is essentially a model of procedural knowledge (i.e., the 'how' of fraction arithmetic) and does not address issues of conceptual knowledge. FARRA specifies the conditions that are present in a fraction arithmetic problem for a procedure to be selected and executed. However, the selection and execution of the algorithmic step for reduction has not been implemented in FARRA. To supplement the FARRA model, I describe a model of whole-number arithmetic, the identical elements model proposed by Timothy Rickard (Rickard, 2005) and use the model to show how identifying common factors of two numbers might be associated to the reduction procedure.

In Chapters 3 to 6, I present data from the studies that address the questions 
posed earlier about reduction. Chapter 3 addresses the question "What fraction knowledge profiles are observed for adults?" with data collected from an online study that assessed participants' knowledge of fraction concepts and procedures, fraction arithmetic, and participants' ability to perform simple whole-number arithmetic. Scores on each assessment and responses on fraction arithmetic problems were compared. Responses from assessments of conceptual and procedural knowledge were used to profile solvers understanding of fractions. Chapter 3 also shows how people in the two profiles performed on single-digit arithmetic problems, provides evidence that adults' can be clustered into two groups of conceptually- and procedurally-stronger solvers, and discusses the strengths and weaknesses of each group of solvers with particular reference to their understanding of fraction concepts and procedures.

Chapter 4 addresses three questions: "What factors cue adults to select strategies as they perform fraction arithmetic?" Chapter 4 extends previous research on fraction arithmetic, showing the similarities and differences between the performance of adults and middle-school student in fraction arithmetic and strategy selection. Chapter 4 also provides some initial insight into the characteristics of fraction problems that cue adults to select inappropriate strategies and highlights the importance of problem characteristics and solvers' fraction knowledge for strategy selection.

Chapter 5 addresses the question: "What are the problem characteristics and solver profiles that facilitate solvers to reduce fractions?" In Chapter 5, I present data collected from 59 participants who also took part in the online study. These participants came into the lab and were presented with fraction arithmetic problems. I collected and analyzed verbal and written protocols to identify the frequency of 
reduction across fraction arithmetic problems and whether solvers reduced fractions before or after they obtained a solution. Chapter 5 also addresses the question of whether individual differences in conceptual and procedural knowledge influence the frequency with which reduction is selected and builds upon the findings of Chapter 4 by providing evidence that problem characteristics that cue previously identified strategies also cue reduction selection.

Chapter 6 addresses the question "Are reducible fraction multiplication problems solved more accurately and quickly than non-reducible problems?" I chose to focus on fraction multiplication because it requires the least number of steps to generate solutions and also because reduction is a useful though not essential strategy to help solvers generate solutions (see Chapter 2). Participants who provided protocols solved multiplication problems with reducible and non-reducible fraction multiplication problems. Participants' responses, accuracy and latencies were compared to determine whether solvers select reduction to perform fraction multiplication when possible and whether the use of reduction assists solvers to generate solutions quickly and effectively.

In Chapter 6, I also addressed the question "Where do solvers focus their attention when they multiply fractions?" Ocular measures from 24 participants who provided protocols were collected for reducible and non-reducible fraction multiplication problems. Besides accuracy rates, responses and latencies, gaze trajectories were also obtained to address whether there were individual differences in attention as solvers multiplied fractions.

In Chapter 7, I provide the general discussion to my dissertation and address the potential impact of my findings. I describe how to integrate the fraction reduction algorithm into FARRA to reflect conceptual knowledge and the procedures used by 
adults to select strategies. I also address limitations to the study and some solutions to the limitations which can be addressed in future research. 
Table 1.1. Overview of questions that were addressed for each experiment and the methods that were used

\begin{tabular}{|c|c|c|c|}
\hline Study/Experiment & Questions & Method & Dependent Measures \\
\hline 1 & $\begin{array}{l}\text { - What fraction knowledge profiles are observed for } \\
\text { adults? } \\
\text { - What factors cue adults to select strategies as they } \\
\text { perform fraction arithmetic? }\end{array}$ & $\begin{array}{l}\text { Participants were recruited } \\
\text { through an online study. They } \\
\text { completed fraction knowledge } \\
\text { assessments, basic arithmetic } \\
\text { problems, and fraction } \\
\text { arithmetic problems. }\end{array}$ & $\begin{array}{l}\text { - Scores on each of the } \\
\text { conceptual and } \\
\text { procedural } \\
\text { assessments and } \\
\text { responses on fraction } \\
\text { arithmetic problems }\end{array}$ \\
\hline $\begin{array}{l}2 \& 3 \\
\text { Part I }\end{array}$ & $\begin{array}{l}\text { What are the problem characteristics and solver } \\
\text { profiles that facilitate solvers to reduce fractions? }\end{array}$ & $\begin{array}{l}\text { Participants were provided } \\
\text { with fraction arithmetic } \\
\text { problems and asked to provide } \\
\text { written and verbal protocols. }\end{array}$ & $\begin{array}{l}\text { - The frequency of } \\
\text { reduction procedures }\end{array}$ \\
\hline $\begin{array}{l}2 \& 3 \\
\text { Part II }\end{array}$ & $\begin{array}{l}\text { Are reducible fraction multiplication problems } \\
\text { solved more accurately and quickly than non- } \\
\text { reducible problems? }\end{array}$ & $\begin{array}{l}\text { - Participants solved reducible } \\
\text { cross-reducible and non- } \\
\text { reducible fraction } \\
\text { multiplication problems }\end{array}$ & $\begin{array}{l}\text { - Accuracy rates and } \\
\text { latencies and ocular } \\
\text { measures }\end{array}$ \\
\hline $\begin{array}{l}3 \\
\text { Part II }\end{array}$ & $\begin{array}{l}\text { - Where do solvers focus their attention when they } \\
\text { multiply fractions? }\end{array}$ & $\begin{array}{l}\text { - Participants solved reducible } \\
\text { cross-reducible and non- } \\
\text { reducible fraction } \\
\text { multiplication problems }\end{array}$ & $\begin{array}{l}\text { - Accuracy rates, } \\
\text { latencies and ocular } \\
\text { measures }\end{array}$ \\
\hline
\end{tabular}




\section{CHAPTER 2: BACKGROUND AND LITERATURE REVIEW}

\section{Background: Fractions and Fraction Arithmetic}

A fraction consists of two numbers, separated by a fraction bar or viniculum. The number above the fraction bar is called the numerator and the number under the bar is called the denominator. As for whole-number arithmetic, there are four fraction arithmetic operations: addition (e.g., 5/6 +2/3), subtraction (e.g., 5/6 - 2/3), multiplication (e.g., $5 / 6 \times 2 / 3$ ) and division (e.g., $5 / 6 \div 2 / 3$ ). All fraction arithmetic problems can be solved by applying rote procedures - that is, for any given fraction arithmetic problem, the application of algorithmic steps in a specific sequence will always lead to the correct solution. These procedures are described below.

\section{Fraction Addition and Subtraction}

To solve fraction addition and subtraction problems, the first step is to convert one or both fractions into equivalent fractions, such that both fractions have equal denominators. Once fractions of equal denominators have been obtained, the numerators of the fractions are either added or subtracted to obtain the numerator of the solution, while the denominator is retained in the solution. Figure 2.1 illustrates the steps required to solve a fraction addition problem.

Figure 2.1. Fraction Addition

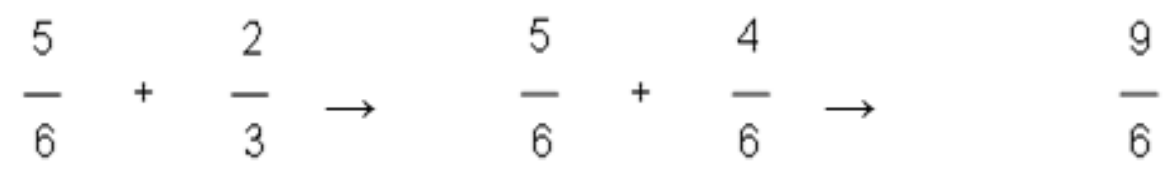

To solve the problem $5 / 6+2 / 3$, the second fraction $2 / 3$ is converted to $4 / 6$ to obtain a fraction pair with equal denominators $5 / 6+4 / 6$. The numerators of the two fractions are then added, while the denominator is retained to obtain the solution $9 / 6$. 
Likewise, in Figure 2.2, which illustrates a fraction subtraction problem, $5 / 6-2 / 3$ is solved by converting the fraction on the right to $4 / 6$ to obtain fractions with equal denominators $5 / 6-4 / 6$. The numerator of the second fraction is then subtracted from the numerator of the first fraction to obtain the solution of the denominator while the denominator is retained to obtain the solution $1 / 6$.

Figure 2.2. Fraction Subtraction

$$
\frac{5}{\frac{6}{6}}-\frac{2}{3} \rightarrow \frac{5}{6}-\frac{4}{6} \rightarrow \frac{1}{6}
$$

\section{Fraction Multiplication}

When two fractions are multiplied, the numerator of the solution is obtained by multiplying numerators of both fractions. Likewise, the denominator of the solution is obtained by multiplying the denominators of both fractions. Figure 2.3 illustrates a fraction multiplication problem. To solve the problem $4 / 6 \times 2 / 3$, the numerator of the two fractions are multiplied $4 \times 2$ to obtain the numerator of the solution 8 , and the denominator of the two fractions $6 \times 3$ are multiplied to obtain the denominator of the solution 18 . The solution is therefore $8 / 18$. I call the former procedure numerator multiplication, and the latter procedure denominator multiplication.

Figure 2.3. Fraction Multiplication

$$
\frac{4}{6} \times \frac{2}{3} \rightarrow \frac{4 \times 2}{6 \times 3} \rightarrow \frac{8}{18}
$$

\section{Fraction Division}

When dividing two fractions, inversion of the second fraction is required. 
During inversion, the numerator and denominator of the second fraction are switched before numerator multiplication and denominator multiplication are applied. Figure 2.4 illustrates a fraction division problem. To solve $5 / 6 \div 2 / 3$ the second fraction $2 / 3$ is inverted to obtain the fraction $3 / 2$. Numerator and denominator multiplication are then applied on the first and inverted fractions $5 / 6 \times 3 / 2$ to obtain the solution $15 / 12$.

Figure 2.4. Fraction Division

$$
\frac{5}{6} \div \frac{2}{3} \rightarrow \frac{5}{6} \times \frac{3}{2} \rightarrow \frac{5 \times 3}{6 \times 2} \rightarrow \frac{15}{12}
$$

\section{Other Fraction Procedures}

In addition to the knowledge of how to apply the required algorithmic steps to solve arithmetic problems, several other procedures are also useful, though not necessary for aiding problem solvers to obtain solutions - namely reduction, crossreduction, and conversion of mixed numbers to fractions and fractions to mixed numbers.

Conversion of fractions to mixed numbers. After performing fraction arithmetic problems, the numerator of a fraction may be larger than the denominator (e.g., Figures 2.1 and 2.4). Under these conditions, the fraction can be converted to a mixed number. Conversion of a fraction to a mixed number involves first dividing the numerator by the denominator. The remainder after the division is the solution of the numerator while the denominator of the fraction is retained. For example, the fraction, $3 / 2$ is converted into a mixed fraction by first dividing 3 by 2 . The quotient of the division procedure is " 1 " with a remainder of 1 . The quotient " 1 " is the whole number associated with the mixed fraction, the remainder 1 , is the numerator of the mixed fraction and the denominator 2 is retained as the denominator of the mixed fraction. Conversion of the fraction $3 / 2$ results in the mixed number $11 / 2$. 
Conversion of mixed numbers to improper fractions. The procedure of converting mixed numbers to fractions involves first multiplying the whole number of the mixed fraction by the denominator, then adding the result to the numerator of the mixed number to obtain the numerator of the improper fraction, while the denominator is retained. For example, the mixed number $1 \frac{1}{2}$ is converted to a fraction by first multiplying the whole number and the denominator $(1 \times 2)+1$ to obtain the numerator of the fraction 3 . The denominator of the mixed fraction is retained as the denominator in the fraction 2 . Hence the mixed number $1 \frac{1 / 2}{2}$ is converted into the equivalent fraction $3 / 2$.

Reduction. Reduction of a single fraction involves dividing both the numerator and the denominator of the fraction by a common factor. As shown in Figure 2.5 , the fraction multiplication problem $4 / 6 \times 2 / 3$ can also be solved by reducing the fraction $4 / 6$ to $2 / 3$ as both 4 and 6 have the common factor 2 to obtain the reduced problem $2 / 3 \times 2 / 3$ before applying numerator multiplication and denominator multiplication to obtain the solution $4 / 9$. Note that reduction is not necessary to obtain a solution although it may be conventional or required under some conditions. It is equally correct to multiply $4 \times 2$ and $6 \times 3$ and answer $8 / 18$. Alternatively, solver could reduce the fraction $4 / 6$ to $2 / 3$ to obtain the simplified problem $2 / 3 \times 2 / 3$ and multiply the numerators and denominators to obtain the solution $4 / 9$ or multiply numerators and denominators of the non-simplified problem to obtain $8 / 18$ then reduce the solution to obtain the solution $4 / 9$.

Cross-reduction. When multiplying fractions, a variant of fraction reduction, cross-reduction allows reducing a numerator of one fraction and the denominator of the second fraction by a common factor. 
Figure 2.5. Reduction

$\frac{4}{6} \times \frac{2}{3} \rightarrow \frac{8 \times 2}{8 \times 3} \times \frac{2}{3} \rightarrow \frac{2 \times 2}{3 \times 3} \rightarrow \frac{4}{9}$

As shown in Figure 2.6, cross-reduction can be applied to the fraction multiplication problem $4 / 6 \times 2 / 3$. The denominator 6 in the first fraction shares a common factor with the numerator 2 in the second fraction. The numeratordenominator pair can be reduced by a factor of 2 , resulting in cross-reduction of the problem to $4 / 3 \times 1 / 3$. Numerator multiplication and denominator multiplication are then applied to obtain the solution 4/9.

Figure 2.6. Cross-Reduction

$$
\frac{4}{6} \times \frac{2}{3} \rightarrow \frac{2 \times 2}{2 \times 3} \times \frac{2}{3} \rightarrow \frac{2 \times 2}{3 \times 3} \rightarrow \frac{4}{9}
$$

Fraction reduction is a procedure that is useful for some types of fraction problems, but not for others. When people multiply or divide fractions, they can use reduction to simplify pairs of large numerator-denominator components and facilitate the arithmetic. For example, in the problem $6 / 10 \times 1 / 3$, the first fraction contains a reducible operand and thus the problem can be simplified to $3 / 5 \times 1 / 3=1 / 5$. This problem is also cross-reducible, in that the numerator of the first operand and the denominator of the second operand share a common factor, so that the problem could be simplified to $2 / 10 \times 1 / 1$. For multiplication and division, therefore, reduction may be a useful step in managing computational complexity.

In contrast, reduction is less often applicable to addition and subtraction. For these operations, reduction is sometimes useful for different denominator problems with a reducible fraction if it allows the fraction to be simplified to a problem with 
equal denominators (e.g., 10/30 $+2 / 3=1 / 3+2 / 3$ ). However, for addition and subtraction, the focus is typically on finding equal denominator fractions. Reduction is not relevant for fraction addition or subtraction problems with equal denominators unless it is applied to both operands. For example, for the problem, 20/30+4/30, reducing one of the fractions in the problem, that is, $2 / 3+4 / 30$ or $20 / 30+2 / 15$, does not allow the solver to apply the correct algorithm of adding numerators for fractions with common denominators.

In the next sections, I review the literature on fraction arithmetic to examine the factors that make fraction arithmetic difficult and how conceptual knowledge of fractions is related to knowing the procedures for fraction arithmetic. I will describe a recently developed model of fraction arithmetic by Braithwaite et al. (2017) that I will use as a framework to describe how the steps used to perform fraction have been implemented in a computer model. However, this model does not include an algorithmic step for fraction reduction. The identification of common factors is a prerequisite to perform fraction reduction. Hence, I will describe a model by Rickard (2005) that describes how factorization is performed for whole numbers (i.e., the identical elements model). I will combine elements from the models proposed by Braithwaite et al. (2017) and Rickard (2005) to develop a framework for identifying individual differences in selection and execution of reduction procedures.

\section{Literature Review}

\section{What makes Fraction Arithmetic Difficult?}

Lortie-Forgues, Tian, and Siegler (2015) provide a non-comprehensive list of what makes fractions difficult to learn. Some of these reasons are relevant for why fraction arithmetic problems are difficult to solve, even for adults. First, fraction arithmetic requires knowledge of many distinct procedures including: (a) knowing 
how to perform the four basic arithmetic operations (i.e., addition, subtraction, multiplication and division), (b) knowledge of the procedures that are needed to solve each type of fraction arithmetic operation, and (c) the knowledge of the procedures for obtaining equivalent fractions, reduction of fractions, and converting fractions to mixed numbers or mixed numbers to fractions. Fraction arithmetic also requires conceptual knowledge about when and why to apply certain procedures (e.g., the conditions under which algorithmic steps such as numerator multiplication should be applied).

Second, there is a lack of clarity about why certain steps in fraction arithmetic are performed. The sequence of steps to solve a fraction problem for any given operation can be applied in a set order that allows correct solutions to be obtained successfully without the knowledge of why each step is performed. Application of procedures requires procedural knowledge, whereas understanding why the procedure works requires a conceptual understanding of fractions. For example, Lortie-Forgues et al. (2015) highlight learners' difficulty in understanding why equal denominators are needed for adding or subtracting fractions, but not for multiplying and dividing fractions; why denominators can be multiplied during fraction multiplication, but not when adding or subtracting fractions; and why inversion of the second fraction in a fraction pair is necessary when dividing fractions.

Third, even after attaining sufficient knowledge to solve fraction arithmetic problems efficiently and accurately, problems involving fractions still require more effort to process than an equivalent problem with whole numbers. A typical fraction has two components and requires more cognitive resources to encode and process than a single number. Pairs of fractions comprise four components, hence arithmetic problems with fraction pairs such as $4 / 2+6 / 3$ require more cognitive resources to 
process than the equivalent number problem $2+2$ (Siegler et al., 2013). Furthermore, maintaining task-relevant fraction components and retaining answers from previously completed steps require working memory resources (Hecht, 1998). Planning and executing subsequent steps also entail that solvers use cognitive resources for selecting and executing uncompleted algorithmic steps. The ability to retrieve arithmetic facts easily from memory also appears to be important for fraction arithmetic, as it helps students find common denominators in fraction problems, freeing up cognitive resources for other tasks such as performing operations on numerators (Hansen et al., 2015). Consistent with these accounts, working memory is correlated with individual differences in fraction arithmetic, even after other relevant variables have been statistically controlled (Hecht \& Vagi, 2010; Jordan et al., 2013; Siegler \& Pyke, 2013).

\section{Conceptual Knowledge, Procedural Knowledge, and Reduction}

Knowledge of when and how to apply procedures is essential in solving fraction arithmetic problems, and thus studying procedural knowledge alone is insufficient for full understanding of why people have difficulty solving fraction arithmetic problems (Rittle-Johnson \& Star, 2007; cf. Braithwaite et al., 2017). Problem solvers could automatically apply procedures in a rote fashion for all problems, obtain the correct solution, and yet not have a conceptual understanding of why applying the procedure leads to a correct solution (Siegler \& Lortie-Forgues, 2015). The ability to master procedures in fraction arithmetic could also coexist along with inaccurate conceptual knowledge (Byrnes \& Wasik, 1991). These difficulties show that even if people are able to apply procedures automatically, they also require conceptual knowledge of fractions to perform fraction arithmetic.

Rittle-Johnson and Schneider (2015) proposed that conceptual and procedural 
knowledge of mathematics is acquired bi-directionally. In this framework, conceptual and procedural knowledge develop iteratively. An increase in understanding of one type of knowledge supports the acquisition of the other type of knowledge, which in turn, facilitates acquisition of the first type of knowledge and so on (Rittle-Johnson, 2017; Rittle-Johnson \& Schneider, 2015; Rittle-Johnson, Schneider, \& Star, 2015; Rittle-Johnson, Siegler, \& Alibali, 2001; Rittle-Johnson \& Star, 2007).

The bidirectional framework has also been applied to fraction knowledge. (Bailey, Hansen, \& Jordan, 2017). Conceptual and procedural knowledge of fractions are learned in a way such that understanding of one category of fraction knowledge is used as a foundational base for understanding of the other category of fraction knowledge, which in turn further facilitates understanding in the first category of knowledge. Proponents of the bidirectional view further argue that procedural knowledge of fractions alone is insufficient to understand the difficulties and confusion that problem solvers experience when selecting and executing fraction arithmetic procedures and the requisite algorithmic steps.

Siegler and Lortie-Forgues (2015) reported that children sometimes have conceptual understanding of fractions, but do not use that knowledge when solving fraction arithmetic. At other times, children seem to lack the relevant conceptual knowledge to solve fractions. For example, Siegler and Lortie-Forgues reported that sixth and eighth graders recognize that the sum of two fractions is larger than the value of either fraction, but yet respond $2 / 4$ to the fraction problem $1 / 2+1 / 2$. These findings suggest that procedural knowledge and the ability to select and implement procedures to perform fraction arithmetic develops and is represented separately from conceptual knowledge (Braithwaite et al., 2017; Siegler \& Lortie-Forgues, 2015). In research on fraction knowledge, third and fourth graders showed large 
variability in answers when they were asked to perform fraction arithmetic problems (Siegler \& Pyke, 2013). When children were presented with fraction arithmetic problems that required the same operation, they produced answers that appeared to have been obtained using different algorithms (Siegler \& Pyke, 2013; Siegler, Thompson \& Schneider, 2011), suggesting that the required fraction arithmetic operation might not always cue the required set of procedures appropriately. Variability in the steps used to perform fraction arithmetic could also result from individual differences in fraction knowledge.

Fraction reduction may draw on conceptual fraction knowledge because reduction requires the understanding of fraction equivalence (i.e., $7 / 14=1 / 2$ ). Fraction reduction may draw on procedural knowledge because solvers can use the algorithmic steps of reducing any numerator-denominator pairs that share a common factor. The ambiguity in the types of knowledge that contribute to the use of reduction raises the question of whether either type of fraction knowledge alone is sufficient or whether both procedural and conceptual knowledge of fractions are required to perform reduction appropriately and efficiently.

Crooks and Alibali (2014) have compiled a set of principles that demonstrate conceptual knowledge in the general domain of mathematics. These principles include: equivalence, the knowledge that two sides of a math equation represent the same quantity; cardinality, the knowledge that when counting discrete objects, the numerical label that is assigned to the final object represents the number of objects; and inversion, the knowledge that two inverse arithmetic operations when applied to the same arithmetic problem result in no change to the arithmetic problem. Of these three principles, equivalence is particularly relevant to the use of reduction to solve problems. Reduction involves the knowledge that different fractions can have the 
same value. The conceptual knowledge that two fractions are equivalent appears to be a requisite for knowing when reduction can be applied. However, reduction does not necessarily have to be applied to solve all problems that contain a reducible fraction.

Crooks and Alibali (2014) noted that in mathematics, the conceptual knowledge of equivalence is least clearly defined, but rather described in terms of how two pieces of knowledge are connected (i.e., they have the same value). In fraction arithmetic, the knowledge of the conditions that are necessary to apply a procedure appropriately can best be described as a "gray area" between conceptual and procedural fraction knowledge. Byrnes (1992) suggested that knowledge of when to select a sequence of algorithmic steps is a type of procedural knowledge. However, in a review on the definitions of conceptual knowledge, Crooks and Alibali (2014) identified that the knowledge of facts and principles to execute algorithmic steps falls under the gamut of conceptual knowledge - that is the understanding of the conditions that need to be satisfied before an algorithm can be applied. The gray area that marks the transition between knowledge of a fact or principle that underlie when a procedure can be used and the sequence of steps that comprise a procedure suggest that most fraction problems rely upon both procedural and conceptual knowledge. The question of whether adults show different strategic profiles in their fraction arithmetic understanding has not been addressed.

\section{The Role of Reduction}

In this dissertation, I will focus on reduction and cross-reduction in fraction arithmetic. Understanding how and when people use reduction is useful for understanding how fractions are understood and processed. First, knowledge of reduction involves a reorganization of structural knowledge about fractions to accommodate the understanding that two or more fractions can have the same value 
despite physically different appearances. Second, applying a cross-reduction procedure involves the conceptual understanding that cross-reduction does not change the value of the solution, despite changing the components of two separate fractions that need to be multiplied. Third, reduction procedures can be very useful for multiplication and division of fractions. For example, $7 / 9 \times 4 / 8$ requires problem solvers to multiply the numerators $7 \times 4$ and $9 \times 8$, calculations that the average solver will find moderately demanding (LeFevre, Bisanz, Daley, Buffone, Greenham, \& Sadesky, 1996). Using reduction on the numerator 4 and the denominator 8 allows the sequence of computations to be reduced to $7 \times 1$ and $9 \times 2$. In general, problems with larger operands are solved more slowly and less accurately than problems with larger operands (see Zbrodoff \& Logan, 2005 for a review of the problem-size effect) and thus reducing the problem has computational advantages. Fourth, reducing fractions in the context of fraction addition and subtraction may occasionally prove detrimental because the correct step involves ensuring that denominators of fractions are identical before numerators can be added or subtracted.

Finally, despite certain advantages in multiplication and division problems, reduction may not always be the fastest or simplest procedure if solvers can perform fraction multiplication and division problems without resorting to reduction, because the execution of rote procedures to solve fraction arithmetic problems typically require fewer steps than fraction problems that can be reduced. For example, the fraction problem $2 / 4 \times 3 / 5$ can be solved using two steps: numerator multiplication and denominator multiplication. Reducing $2 / 4$ to $1 / 2$ to obtain the simpler problem $1 / 2 \times 3 / 5$ requires an additional step. Reduction is less efficient if solvers can directly multiply the numerators $2 \times 3$ and denominators $4 \times 5$ of the unreduced fraction. Knowledge of reduction also involves knowing the types of problems when reduction 
is not useful and when reduction is beneficial. Understanding how and when people use reduction is a way to bridge conceptual and procedural understanding of fractions because reduction draws upon both conceptual and procedural knowledge.

To date, current theories that describe algorithmic steps for fraction arithmetic problems have not implemented reduction procedures within their framework. This dissertation will, in part, address the gap in the literature by providing some insight into how reduction procedures can be incorporated into current theories of fraction arithmetic.

\section{Current Theories Relevant to Fraction Arithmetic}

In this section, I review how solvers might select and execute the steps that are used in fraction arithmetic. I will first draw upon ideas from a computational model for solving fraction arithmetic problems recently developed by Braithwaite et al. (2017). Braithwaite et al. implemented algorithmic steps to solve fraction arithmetic problems in a computational model named Fraction Arithmetic Reflects Rules and Associations (FARRA). The algorithmic steps implemented in FARRA were based on data collected from students in grades 6 to 8 as they solved fraction arithmetic problems (Siegler, Thompson \& Schneider, 2011; Siegler \& Pyke, 2013). FARRA simulates the selection and execution of algorithmic steps in fraction arithmetic, as well as the types of errors that result from incorrect selection and execution of algorithmic steps. FARRA also successfully simulates patterns of accuracy rates, patterns of procedure use, and the types of errors commonly observed in students for each fraction arithmetic operation (Siegler \& Pyke, 2013). The algorithms implemented in FARRA are suitable as a model for the selection and execution of procedures that might be used by young adults to solve fraction arithmetic problems. However, as pointed out by Braithwaite et al. (2017), FARRA does not explicitly 
represent conceptual knowledge. Accordingly, FARRA cannot use information about fraction equivalencies to implement reduction procedures. Hence, the procedure for selecting and executing fraction reduction has not been implemented in FARRA.

FARRA is a computational model that models the procedures that people use to solve fraction arithmetic. To solve fraction arithmetic problems, FARRA applies the steps for each type of fraction arithmetic operation. These steps are represented in FARRA in the form of condition-action rules. Fraction reduction has not been implemented in FARRA. Fraction reduction involves knowledge of factoring - that is, the ability to identify common factors for a whole number. The inclusion of factorization procedures into FARRA is important to understand how people might perform fraction arithmetic. As a precursor to including the reduction procedure into a model for fraction arithmetic and FARRA, I will describe how people retrieve facts to solve problems involving factorization by drawing upon elements from the identical elements model of whole-number arithmetic (Rickard, Healy \& Bourne, 1994; Rickard \& Bourne, 1996; Rickard, 2005).

In this section, I will first discuss experiments that were conducted to identify the types of errors that children generate and the problems that give rise to these errors. I will then use ideas from FARRA and the identical elements model to describe strategies that problem solvers can use to solve fraction arithmetic problems and how the rules implemented in FARRA might generate systematic errors for fraction arithmetic problems. I will use these ideas as a framework to describe individual differences in selection and execution of algorithmic steps that adults might use to solve fraction arithmetic problems, with a focus on fraction multiplication. I will then provide the rationale for two experiments that examine which strategies are commonly selected by problem solvers with different levels of fraction knowledge. 
Errors generated by middle school solvers. Siegler, Thompson and Schneider (2011) presented 6th and 8th grade learners with fraction arithmetic problems and found that learners were most accurate with fraction addition and subtraction and least accurate with fraction division. The most common strategy error that learners made was to treat the numerators and denominators of each fraction as if they were independent and apply the indicated arithmetic operation to each component. This type of error was commonly observed when children added or subtracted fractions. For example, given the problem $3 / 5+1 / 2$ solvers added the numerators $3+1=4$ and the denominators $5+2=7$ to generate the solution $4 / 7$. Similarly, for the subtraction problem $3 / 5-1 / 2$, a common error was to subtract the value of the numerator in the second fraction from the numerator of the first fraction 3 $-1=2$; and the value of the denominator in the second fraction from the denominator of the first fraction $5-2=3$ to generate the solution $2 / 3$. Siegler and his colleagues called these errors independent whole number errors because solvers treated each number in the numerator and denominator as separate whole number entities without considering the properties of numbers as components of a fraction.

Another common error - the wrong fraction operation error - involved selection and application of an incorrect procedure for maintaining the denominator while applying the required operation on the numerator (Siegler et al., 2011). This error was observed in fraction multiplication and division. For example, when presented with the problem $3 / 5 \times 2 / 5$, children multiplied the numerators $3 \times 2$ and maintained the common denominator 5 to generate the solution $6 / 5$. These strategy errors and the types of problems for which they are observed have been replicated in later studies (Braithwaite et al., 2017; Braithwaite \& Siegler, 2018; Siegler \& Pyke, 2013). 
A model of fraction arithmetic (FARRA). FARRA uses production rules to implement selection and execution of algorithmic steps that are used to solve fraction arithmetic problems. The production rules are assumed to model the types of processes involved when humans select and execute algorithms for performing fraction arithmetic. These production rules are implemented within FARRA and mirror the correct and incorrect steps middle-school children apply when performing fraction arithmetic (Siegler et al., 2011; Siegler \& Pyke, 2013). FARRA learns to apply rules based on the principle that people identify statistical associations between problem characteristics and solutions to guide their selection of procedures. However, in the context of mathematics, learning to apply procedures based on identifying statistical associations occasionally leads to selecting an incorrect production rule. This rule deviates slightly from the correct production rule, but nevertheless leads to an error when the rule is applied. When FARRA is presented with fraction arithmetic problems, the model identifies statistical associations between presented problems and the correct solution to learn rules for generating solutions. These rules are then applied for other fraction arithmetic problems presented to the model.

When FARRA is presented with a problem, FARRA maintains a representation of the problem. The representation comprises the arithmetic operation, as well as the numerator and denominator of each fraction. FARRA also maintains a list of the goals and sub-goals that are incorporated into FARRA's memory while solving the problem and the results of intermediate computations while trying to attain these goals.

Each production rule is a "modular component of knowledge" that consists of a condition-action pair (Braithwaite et al., 2017, p. 7). The condition part of the rule specifies the criteria that need to be satisfied before the action part of the rule is fired. 
For example, a rule when adding fractions is "if denominators are equal, then add numerators". In this rule. the condition is "denominators are equal". If this condition is satisfied the action "add numerators" is executed. The firing of each production rule causes changes to each representation (e.g., the numerator) and/or adds new sub-goals to working memory, which in turn determines which production rule fires next.

Production rules are selected and fired iteratively until a solution (the goal) is obtained.

There are two types of production rules: strategy rules and execution rules. Strategy rules are the selection of algorithmic steps that need to be executed to attain the solution to the problem. Initially, selection of strategy rules is determined by the fraction arithmetic operation (i.e., addition, subtraction, multiplication or division) that needs to be performed. Initial selection of strategy rules creates sub-goals, which are added to the list of goals that the model needs to attain. Execution rules are the implementation of each algorithmic step, which involves obtaining a solution for each created sub-goal.

Mal-rules are rules that deviate in a small way from the correct rule, but if implemented will generate an incorrect solution (Brown \& VanLehn, 1980; Payne \& Squibb, 1990). Braithwaite et al. (2017) categorize two types of mal-rules in the context of fraction arithmetic. Strategy mal-rules are a deviation of the algorithmic steps that form correct strategy rules. Similarly, execution mal-rules reflect a failure to apply one or more of the execution rules correctly. Execution mal-rules could occur either when a correct strategy rule or mal-rule is selected (see Appendix A for strategy and execution rules and mal-rules in the current version of FARRA). For purposes of this dissertation, I will focus on correct strategy and execution rules.

To illustrate, I provide an example of how FARRA solves fraction 
multiplication. See Table 2.1 for the relevant selection and execution rules to obtain the correct solution for fraction multiplication. The complete set of rules implemented within FARRA can be found in Appendix A.

In the first iteration, the condition "operation is $\times$ " is satisfied and the strategy Rule "Correct Mult" fires. The action for the rule "Correct Mult" is "Create goals to set denominator and numerator of answer equal to results of performing given operation on operand denominators and numerators respectively". The firing of the rule "Correct Mult" adds two additional goal states that FARRA is required to solve. "Goal 1" and "Goal 2" respectively. Goal 1 sets the denominator of the answer as the result of performing the given operation "multiplication" on the denominators of the fractions in the problem. Goal 2 sets the numerator of the answer as the result of performing the given operation "multiplication" on the denominators of the fractions in the problem.

In iteration 2, the condition "Goal exists to set answer denominator to result of performing given operation on denominators" for the Execution Rule "OperateDenominators" is satisfied. The action for the rule "Operate Denominators" is "Create goal to solve appropriate whole number arithmetic problem". This action creates an additional goal 1a, which is to "solve whole number arithmetic problem for denominators." The firing of the Rule "Operate-Denominators" also removes Goal 1 from the list of goals maintained in FARRA.

In iteration 3, FARRA implements the action required to attain Goal 1a. The denominators of the fractions are multiplied resulting in a change in the problem state maintained in FARRA (i.e., $(1 \times 3) / 16)$.

In iteration 4, the condition "Goal exists to set answer numerator to result of performing given operation on denominators" for the Execution Rule “operate 
numerators" is satisfied. The action for the rule "Operate numerators" is "Create goal to solve appropriate whole number arithmetic problem”. This action also replaces goal 2 with goal 2a - "solve whole number arithmetic problem for numerators."

In iteration 5, FARRA implements the action required to attain Goal 2a. The numerators of the fractions are multiplied resulting in a change in the problem state maintained in FARRA "3/16". After the action performed in iteration 5, there are no additional goal states. FARRA does not perform any further action and FARRA's solution to the multiplication problem $1 / 4 \times 3 / 4$ is " $3 / 16$ ".

The processes that initiate selection and execution of algorithmic steps in FARRA is a model of how solvers perform fraction arithmetic (Braithwaite et al., 2017). When solvers perform fraction arithmetic, they first identify the type of arithmetic operation that needs to be performed. Next, they use the required arithmetic operation as a cue to set goals and select a strategy. Each strategy is composed of a sequence of algorithmic steps, which if applied to fraction components correctly, will eventually generate a solution. Finally, solvers execute the steps upon components of the fraction problem to obtain the solution. For example, when problem solvers solve a fraction multiplication problem, the required operation of fraction multiplication might cue the strategy of denominator multiplication followed by numerator multiplication. To multiply denominators and numerators of fractions, further retrieval of whole number multiplication facts is necessary. Solvers then execute the steps of numerator multiplication and denominator multiplication to obtain the required solution. 
Table 2.1: Firing of correct strategy and execution production rules for the multiplication problem 1/4 $\times 3 / 4$

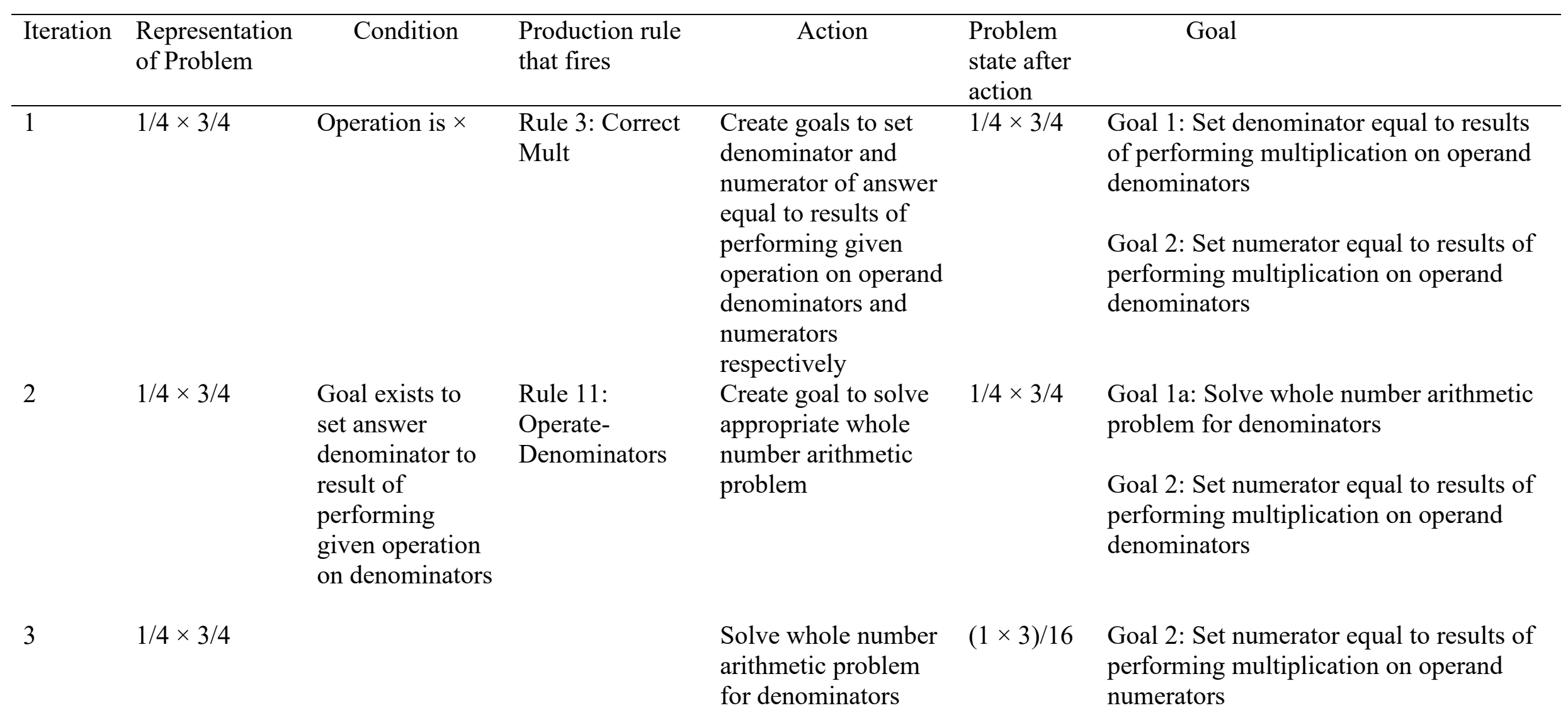


4

Goal exists to Rule 12:

Operate-

Create goal to solve

$(1 \times 3) / 16$

Goal 2a: Solve whole number arithmetic

set answer

Numerators

appropriate whole

number arithmetic problem for numerators

numerators to problem

\section{performing}

given operation

on numerators 
The identical elements model of whole-number arithmetic. To date, the reduction procedure has not yet been implemented in FARRA. However, if solvers do use reduction while performing fraction arithmetic, then the conditions and actions for the production rules need to be specified to implement the reduction algorithm within FARRA. A potential candidate for a framework about how solvers might reduce fractions involves the identical elements model (Rickard, 2005). To date, the revised identical elements model is the only cognitive model that integrates both whole number multiplication and factorization within the same framework. Factorization is a process of identifying the divisors (i.e., a factor) of whole numbers such that the whole number, when divided by a factor will provide another whole number. For example, the whole number 21, has the factors 3 and 7, because when the number 21 is divided by 3 , another whole number 7 is obtained. Likewise, when 21 is divided by 7, the whole number 3 is obtained. Rickard highlights the importance of factorization in reduction as it allows solvers to "perform fractional operations such as reducing to a common denominator" (Rickard, 2005, p. 251).

The revised identical elements model is an extension of an earlier model that could account for multiplication and division but not factorization (Rickard et al., 1994; Rickard \& Bourne, 1996). The revised identical elements model assumes three distinct stages: an early perceptual stage, a central cognitive stage, and a late motor stage. Access to retrieval of facts for solving mental arithmetic problems is assumed to occur exclusively within the central cognitive stage (McCloskey, Caramazza, \& Basili, 1985; McCloskey, 1992). When problem solvers are presented with a mental arithmetic problem, elements of the problem (i.e., operands and operators) are encoded and used as a cue to access and retrieve relevant facts to obtain solutions.

The revised identical elements model posits that multiplication (and division) 
facts are stored as a chunk. Each chunk consists of a number triplet and an arithmetic operation. Two elements of the number triplet and the operation feed-forward to the third member of the triplet - the required answer. For example, “ $3,5, \times$ " feeds forward to the solution " 15 " for a multiplication fact. A reverse link is also concurrently created that associates the solution to the other elements of the chunk for each fact.

The identical model also predicts how people find the common factors " 5 " and "3" when presented with the product of the numbers " 15 ". When children learn multiplication facts, they form an association of links between the product and its factors. The reverse link between the products to its factors is initially weaker than the forward link. However, with sustained practice, the reverse link is strengthened such that the product can also function as a cue to obtain its common factors.

Fraction arithmetic problems can be solved without the need for reducing fractions. However, if reduction is a frequently used step in fraction arithmetic, the steps required for reduction need to be described. The fraction reduction procedure involves steps of identifying whether the numerator and denominator of a fraction shares a common factor, then further executing the additional step(s) of dividing the numerator and denominator by the common factor. However, executing additional steps to reduce a fraction, in addition to rote procedures should incur a latency cost. Thus, there appears to be no benefit to adding additional steps to an algorithm that already leads to a correct solution. I propose, however, that reduction, if used appropriately, simplifies problems. Solvers who use reduction are therefore expected to be more accurate when performing fraction arithmetic. 


\section{CHAPTER 3: STUDY 1}

In Study 1, I first assessed participants' conceptual and procedural knowledge of fractions to identify their fraction knowledge profiles. In previous research Hallett et al. $(2010 ; 2012)$ had shown that Grade 6 students could be profiled into four clusters, whereas Grade 8 students could be profiled into two clusters. However, the ideal number of clusters has not been determined for adults. I collected data in an online format to ensure a large sample size, increase the stability of identified profiles, and provide a pool of people for subsequent experiments. Participants' profiles were used to determine membership for the experimental groups in Study 2. Fraction knowledge profiles identified in Study 1 were also used to determine the optimal number of profiles for adults.

To assess individual differences in conceptual and procedural fraction knowledge in children, Hallett et al. (2012) tested sixth and eighth grade British students' knowledge of fraction concepts and procedures by administering a paperand pencil test of items obtained from the Chelsea Diagnostic Mathematics tests (Brown, Hart, \& Kuchermann, 1984) and from the second National Assessment of Educational Progress (Bezuk \& Cramer, 1989). Hallett et al. categorized participants according to their fraction knowledge by performing a cluster analysis. First, each participant's conceptual score was regressed against his or her procedural score and the standardized residuals were recorded for each participant as the residualized conceptual scale. Then, each participant's procedural scores were regressed against his or her conceptual score, and the standardized residuals were recorded for each participant as the residualized procedural scale. These two steps created measures of conceptual and procedural fraction knowledge that were independent from overall conceptual and procedural scores. Thus, a high score on the residualized conceptual 
subscale does not necessarily indicate a high level of conceptual understanding but indicates a higher than expected level of conceptual understanding given that participant's procedural score. Similarly, a high residualized procedural score indicates that participant did relatively better on procedural than conceptual items.

Hallett et al. (2012) performed a cluster analysis and found four fraction knowledge profiles among sixth grade children. These four strategic profiles included: (a) children who did poorly on both conceptual and procedural fraction knowledge assessment, (b) children who did well on types of knowledge assessments, (c) children who were stronger on procedural than conceptual fraction knowledge, and (d) children who were stronger on conceptual than procedural fraction knowledge. However, by eighth grade, Hallett et al. found that children's understanding of fractions was captured by two profiles. One profile was classified as conceptual solvers, while the other profile was classified as procedural solvers. Conceptual solvers are relatively stronger in their knowledge of concepts such as fraction equivalence to solve fraction-related tasks than in their ability to use procedures. Procedural solvers are stronger in their ability to execute a series of algorithmic steps when confronted with fraction-related tasks than in their ability to use concepts. Note that people who are conceptual solvers are able to use procedures but they perform relatively better on conceptual knowledge items. Likewise, people who are procedural solvers can also use knowledge of fraction concepts for fraction-related tasks. These results suggest that although individual differences in fraction understanding start to emerge by sixth grade, with experience, solvers tend to rely more heavily on either conceptual or procedural knowledge to solve problems involving fractions.

Note that the cluster analysis was performed on residual rather than overall conceptual and procedural scores. Overall conceptual and procedural scores are 
positively correlated with each other, whereas residualized conceptual and procedural scores were negatively correlated with each other. However, the residualized conceptual score is independent from both the overall conceptual and procedural scores and the residualized procedural score is independent from both the overall conceptual and procedural scores. Residualized conceptual and procedural scores indicate relative performance because they indicate performance on each type of measure relative to what should be expected given their performance on the other type of measure. Overall conceptual and procedural scores indicate overall performance because these scores indicate how each participant's overall knowledge of fractions contributes to their overall scores on each measure.

In Study 1, I tested two hypotheses in a sample of adult solvers. These hypotheses are based on inferences of how people's knowledge of fractions may change from grade 8 to university.

Hypothesis 1: Solvers learn to use fraction concepts and procedures iteratively until knowledge of concepts and procedures reach a similar level. They are therefore able to solve both conceptual and procedural questions with equal efficiency. If this hypothesis were correct, I would expect to observe a single cluster for all solvers.

Hypothesis 2: Solvers do not show much change in patterns of performance at grade 8 and university. If this hypothesis is correct, I expect that solvers will be optimally clustered into two profiles. These two profiles should be consistent with the two profiles identified in grade 8 students. Members of one profile, the conceptuallystronger group (i.e., the "more conceptual" group in Hallett et al, 2012), are expected to score relatively better than the other profile on conceptual items but relatively worse on procedural items. In contrast, members of the procedurally-stronger group (i.e., the "more procedural" group in Hallett et al., 2012) are expected to score 
relatively better on procedural but worse on conceptual items.

Previous research demonstrated a correlation between performance on basic arithmetic and knowledge of fraction concepts and procedures (Jordan, Hansen, Fuchs, Siegler, Gersten \& Micklos, 2013). Specifically, Jordan and her colleagues found that calculation fluency - the ease with which third grade children could perform basic arithmetic - predicted their knowledge of fraction concepts and procedures at fifth grade. Furthermore, Jordan et al. (2013) also found that the ease with which children were able to retrieve basic multiplication facts was related to their ability to perform fraction arithmetic. These findings suggest that adults' knowledge of basic arithmetic might also be related to their choice of strategies on fraction arithmetic problems, and their ability to reduce fractions. Therefore, I further introduced assessments of calculation fluency for arithmetic and basic multiplication to determine the impact of basic arithmetic and multiplication on participants' choice of strategies.

\section{Method}

\section{Participants}

Nine hundred and six participants enrolled in first-year undergraduate Cognitive Science and Psychology courses at Carleton University participated in return for course credit. Of this total, 24 participants completed the questionnaire twice. Only the first set of responses for these participants (882 participants) were included in the study.

\section{Materials}

Participants were administered a set of five online measures. The measures are listed in Table 3.1 and described in greater detail in the text. Measures were presented 
in the order listed. Participants typed in their responses in the space provided unless otherwise specified.

Mathematics Background and Interests Questionnaire (MBIQ). On the MBIQ, participants reported their age, sex and educational background. Participants also reported their behaviors when confronted with math and fraction problems (LeFevre et al., 1992). Participants were asked to rate their arithmetic skills (i.e., Please rate your level of basic mathematical skill), fraction skills (i.e., Please rate your level of skill with fractions), and their frequency of types of strategies (i.e., How frequently do you solve math problems such as $3 \times 9$ by switching the numbers around so that the larger number is placed first " $9 \times 3$ ")? on a five-point Likert-type scales (see Appendix B for the complete questionnaire).

Table 3.1. Measures.

Name of Measure Description

(1) The Mathematics Background Information about participants' personal and Interests Questionnaire (MBIQ; and educational background and their LeFevre, Kulak \& Heymans, 1992) behaviours when confronted with math and fraction problems.

(2) Calculation Fluency Test (CFT; Multi-digit arithmetic problems. One page Sowinski, Dunbar \& LeFevre, 2014) each of sixty addition (e.g., $34+17$ ), sixty subtraction (e.g., $47-19)$, and sixty multiplication (e.g., $45 \times 8$ ) problems.

(3) Basic Multiplication Fluency Test Single-digit multiplication problems, one (BMT; developed for this study) set with products of 25 of less (e.g., $4 \times 2$ ), the other with products of 27 or more (e.g., $4 \times 8)$ 
(4) Fraction Knowledge Assessment Assesses participants' conceptual and (FKA; Hallett et al., 2012) procedural understanding of fractions (see text for examples).

(5) Fraction arithmetic problems Assesses participants' ability to solve (Braithwaite, Pyke \& Siegler, 2017). $\quad$ fraction arithmetic problems (e.g., 1/3+ $1 / 4)$

Calculation Fluency Test (CFT). The CFT is divided into three sections. Each section requires participants to solve multi-digit addition, subtraction and multiplication problems (Sowinski et al., 2014). For the addition and subtraction sections, participants are required to add or subtract two-digit numbers. For the multiplication section, participants are required to multiply a two-digit number with a single digit number. Participants are required to solve as many problems within each section in one minute. Participants are also required to solve the problems in the order in which they were presented and not to skip questions. The CFT score for each section obtained by each participant was obtained by calculating the number of correct responses for that section. The total CFT score for each participant was obtained by calculating the number of correct responses for all three sections of the CFT.

Basic Multiplication Test (BMT). The BMT is divided into two sections. Each section consists of problems that require participants to multiply two single-digit numbers within a time limit and section consists of 60 problems. For the first section - BMT (small) -- the smallest correct answer is 4 (i.e., $2 \times 2$ ) and the largest is 25 (i.e., $5 \times 5$ ). For the second section - BMT (large) -- the smallest correct answer is 27 (i.e., $3 \times 9$ ) and the largest is 81 (i.e., $9 \times 9$ ). As with the CFT, participants are 
required to solve as many problems within each section in one minute, in the order in which they were presented and not to skip questions. Participants' scores for the BMT (small) and BMT (large) were the number of correct responses for the respective measure.

Fraction Knowledge Assessment (FKA). The FKA was used by Hallett et al. (2012) to assess children's understanding of fraction concepts and procedures. There are a total of 37 questions; 25 are conceptual items that assess participants' knowledge of the relations between fractions and fraction facts and 12 are procedural items that assess participants' knowledge of how to select and apply algorithmic steps to solve fraction arithmetic problems. Below, I describe each type of question in greater detail. The 37-item FKA is presented in Appendix C.

Conceptual items. The conceptual section of the FKA tested four categories of fraction concepts. These concepts reflected principles that other researchers have identified in studies of the conceptual understanding of fractions (Hecht, 1998; RittleJohnson, Siegler, \& Alibali, 2001). See Table 3.2 for examples of each principle. 
Table 3.2. Principles tested in the conceptual FKA and examples.

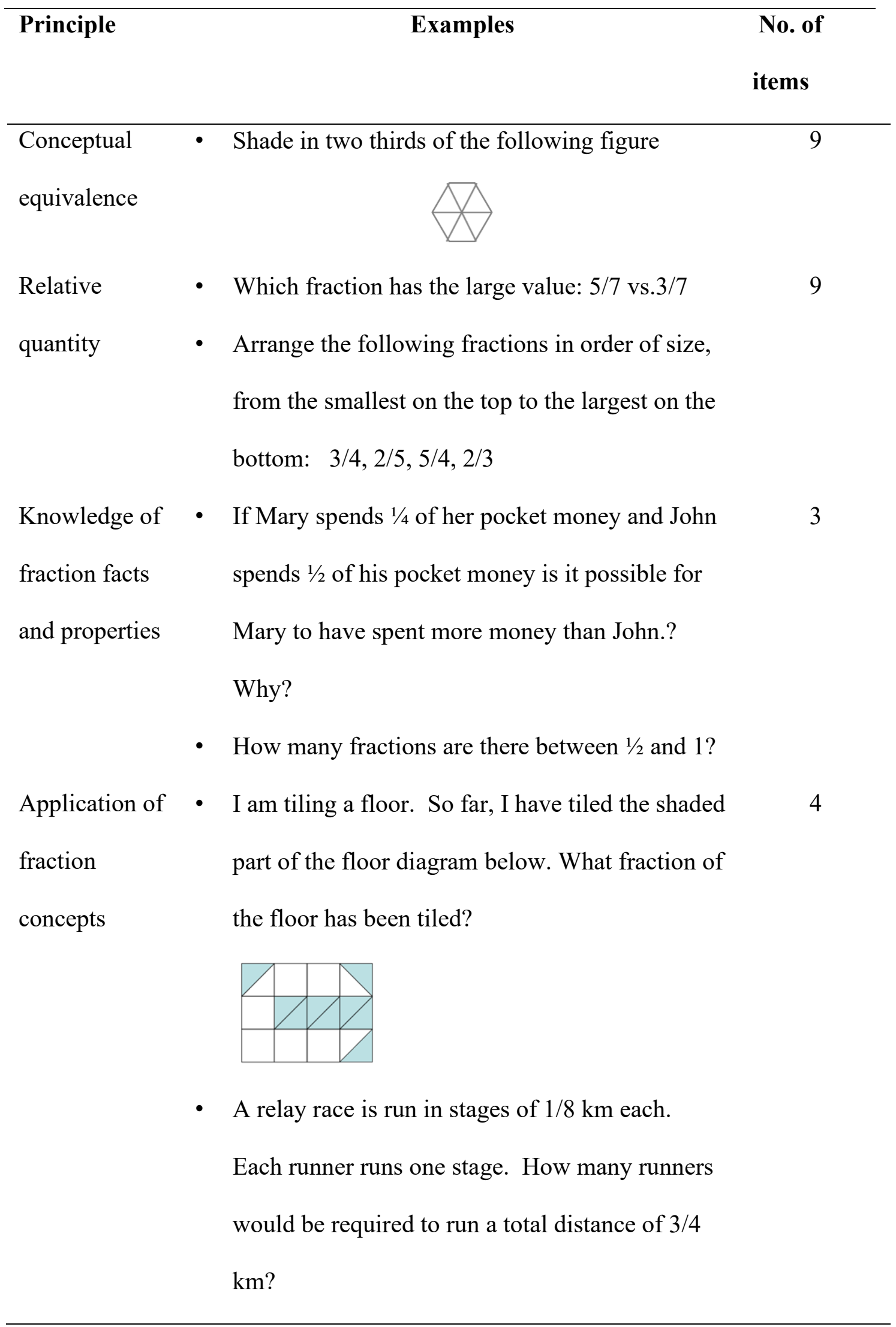


Nine items assess solvers' conceptual understanding of equivalence. These items require participants to shade a figure to ensure that the figure correctly represents the equivalent magnitude as a given fraction. Nine other items reflect relative quantity knowledge. These items require participants to compare two fractions or to arrange fractions in ascending order. These items are conceptual because they test participants' understanding of the relative magnitudes of two or more fractions. Three items tested participants' understanding of fraction facts and properties, such as knowledge of the fraction fact that there are an infinite number of fractions between any two numbers. Four other items assess solvers ability to apply fraction knowledge to a real-life situation. The conceptual section of the FKA was scored out of 25 .

Procedural items. There were 12 procedural items (see Table 3.3 for examples). Procedural items assess solvers' application of an algorithm or procedure taught in middle school. Nine items were fraction arithmetic problems. The other three items require participants to fill in a missing number to ensure that two fractions had equal values. These items were assumed to assess procedural equivalence because they require solvers to apply algorithms to obtain the missing number. The procedural section of the FKA was scored out of 12. Both fraction and decimal answers were scored as correct.

Responses for the FKA were provided in three ways. Firstly, when solvers were required to compare fractions, they were presented with the possible options and selected their responses by clicking on the appropriate box. Secondly, when solvers were required to shade parts of a figure to indicate a fraction, they were presented with an unshaded figure divided into equal parts and clicked on unshaded parts of the figure, which was then shaded red. Thirdly, when solvers were required to fill in an 
answer with a fraction, they filled in their responses using an alpha-numeric keyboard or touchpad on their personal device. Respondents entered the viniculum in fractions with either the "/" or the " $r$ " symbol.

Table 3.3. Examples of procedural items from the Fraction Knowledge Assessment (FKA).

\begin{tabular}{lll}
\hline $\begin{array}{l}\text { Principle } \\
\text { described }\end{array}$ & Examples & Number of items \\
\hline
\end{tabular}

Fraction arithmetic $\quad$ Fraction addition: $4 / 9+7 / 14=? \quad 2$

- Fraction subtraction: $4 / 5-5 / 8=? \quad 3$

- Fraction multiplication: $3 / 5 \times 5 / 12=$ ? 3

- Fraction division: $2 / 3 \div 2 / 6=? \quad 3$

$\begin{array}{lll}\text { Procedural } & -6 / 9=? / 3 & 3\end{array}$

equivalence

Fraction arithmetic problems. Participants solved 24 fraction arithmetic problems: six each of addition, subtraction, multiplication and division problems (see Table 3.4). Participants were scored correctly as long as they gave the correct value in either fractions or decimals. These problems were identical to those used by Braithwaite et al. (2017). 
Table 3.4: Fraction arithmetic problems presented to participants in Study 1

\begin{tabular}{|c|c|c|c|}
\hline Addition & Subtraction & Multiplication & Division \\
\hline \multicolumn{4}{|c|}{ Same Denominator } \\
\hline $3 / 5+1 / 5$ & $3 / 5-1 / 5$ & $3 / 5 \times 1 / 5$ & $3 / 5 \div 1 / 5$ \\
\hline $3 / 5+2 / 5$ & $3 / 5-2 / 5$ & $3 / 5 \times 2 / 5$ & $3 / 5 \div 2 / 5$ \\
\hline $4 / 5+3 / 5$ & $4 / 5-3 / 5$ & $4 / 5 \times 3 / 5$ & $4 / 5 \div 3 / 5$ \\
\hline \multicolumn{4}{|c|}{ Different Denominators } \\
\hline $3 / 5+1 / 2$ & $3 / 5-1 / 2$ & $3 / 5 \times 1 / 2$ & $3 / 5 \div 1 / 2$ \\
\hline $3 / 5+1 / 4$ & $3 / 5-1 / 4$ & $3 / 5 \times 1 / 4$ & $3 / 5 \div 1 / 4$ \\
\hline $2 / 3+3 / 5$ & $2 / 3-3 / 5$ & $2 / 3 \times 3 / 5$ & $2 / 3 \div 3 / 5$ \\
\hline
\end{tabular}

\section{Results}

\section{Exclusion criteria}

Of the 882 participants who completed the survey, 162 participants were excluded (i.e., $82 \%$ retention). Of these, 82 participants $(9.3 \%)$ did not complete the study and $80(9.1 \%)$ scored 0 on the procedural section of the FKA, suggesting that they either were not cooperating or did not understand fraction arithmetic. Although another 201 (22.8\%) participants skipped at least one question in the CFT or BMT, data for these participants were included in the analysis as long as they satisfied the other inclusion criteria.

For the final sample of 720 participants, the median age was 19 years, range of 17 to 47 years. There were 434 women $(60.3 \%)$ and 286 men (39.7\%). Participants were enrolled in a variety of programs, with $44.3 \%$ studying for a Bachelor of Arts, $16.0 \%$ for a Bachelor of Commerce, $10.7 \%$ for a Bachelor of Computer Science, and $17.1 \%$ for a Bachelor of Science. The other $11.9 \%$ were enrolled in other degree 
programs. English was the first language for most participants (77.6\%). The majority of the participants attended school in Canada, both for elementary $(77.5 \%)$ and high school (88.8\%). Most participants had enrolled in their last mathematics course in Grade 12 (76.7\%) or Grade 11 (14.7\%). (See Appendix D for more detailed statistics).

Descriptives. Table 3.5 displays the average completion time (and standard deviation) in minutes for each measure and for the whole online assessment. The time limit was set at one minute for each screen corresponding to each section of the CFT and the BMT. There was a wide range in the time taken to complete the FKA and FARRA measures because this study was done online and there were no time restrictions completion time for the study. Participants were able to do other activities (i.e., using a calculator to obtain answers, or taking a break). Thus, the range of completion times for the FARRA items and the FKA might reflect participants performing other activities rather than their actual time spent to solve problems. Table 3.5: The number of participants $(N)$ that completed each assessment, average time (in min) and standard deviation taken to complete each assessment. Each section of the CFT and BMT had a fixed time limit of 1 min (60s).

\begin{tabular}{lccc}
\hline & $N$ & Median time (min) & Range (min) \\
\hline CFT (addition) & 719 & 1.0 & 1 \\
CFT (subtraction) & 719 & 1.0 & 1 \\
CFT (multiplication) & 719 & 1.0 & 1 \\
BMT (small) & 718 & 1.0 & 1 \\
BMT (large) & 715 & 1.0 & 1 \\
Fraction Arithmetic (FARRA) & 677 & 4 & $1-53$ \\
Fraction Knowledge (FKA) & 720 & 10 & $0.5-22$ \\
Total & 720 & 28 & $4-80$ \\
\hline
\end{tabular}

Table 3.6 shows the mean, standard deviation, and median scores along with the range of scores for participants who generated at least one correct response for the 
mathematical measures. Scores on basic arithmetic problems varied widely, with some participants only being able to solve a single problem, whereas others were able to provide correct solutions for almost all of the sixty problems. Participants' scores for fraction arithmetic and knowledge of fraction procedures also varied widely. Some participants did not provide any correct responses to assessments of procedural knowledge and fraction arithmetic, whereas other participants responded correctly to all the problems in the assessments. In summary, participants' ability to solve basic arithmetic problems and use fraction concepts and procedures to solve problems varied widely.

Table 3.6. Descriptive Statistics for each Mathematical Measure

\begin{tabular}{lrrrr|c}
\hline & Mean & SD & Median & Range & Max \\
\hline CFT (addition) & 9.4 & 4.6 & 9.0 & $1-58$ & 60 \\
CFT (subtraction & 8.7 & 4.3 & 8.0 & $1-58$ & 60 \\
CFT (multiplication) & 6.1 & 4.2 & 6.0 & $1-59$ & 60 \\
\hline BMT (small) & 27.2 & 10.5 & 26.0 & $3-60$ & 60 \\
BMT (large) & 13.7 & 7.7 & 12.0 & $1-60$ & 60 \\
\hline Fraction Arithmetic (FARRA) & 17.1 & 6.8 & 20.0 & $1-24$ & 24 \\
\hline Fraction Knowledge (FKA) - & 17.9 & 4.2 & 18.5 & $6-25$ & 25 \\
Conceptual & & & & & \\
- Conceptual equivalence & 7.1 & 1.9 & 8.0 & $0-9$ & 9 \\
- Relative quantity & 7.8 & 1.4 & 8.0 & $0-9$ & 9 \\
$\bullet \quad$ Fraction Facts & 1.6 & 1.0 & 2.0 & $0-3$ & 3 \\
$\bullet \quad$ Application of concepts & 1.8 & 1.2 & 2.0 & $0-4$ & 4 \\
\hline Fraction Knowledge (FKA) - & 7.1 & 3.4 & 8.0 & $1-12$ & 12 \\
Procedural & & & & & \\
- Fraction arithmetic & 4.8 & 2.8 & 5.0 & $0-9$ & 9 \\
- Procedural equivalence & 2.4 & 0.9 & 3.0 & $0-3$ & 3 \\
\hline
\end{tabular}

Note. Max refers to the maximum possible score for that test or section.

\section{Analysis}

Internal reliability among items for the subscales of the CFT were calculated 
from the scores of all participants who completed all three subscales $(n=685$; Cronbach's alpha $=.87)$. Likewise, internal reliability for the subscales of the BMT were calculated from the scores of all participants who completed both subscales $(n=$ 713; Cronbach's alpha $=.80$ ). Internal reliability for conceptual and procedural FKA was calculated from responses of the participants who scored at least 1 on both FKA scores $(n=720)$. Cronbach's alpha was .82 for the 25 conceptual items and .84 for the 12 procedural items.

Overall, there was high internal reliability (Cronbach's alpha $=0.94)$ among the 24 fraction arithmetic (FARRA) problems calculated from responses of participants who generated at least one correct response on the FARRA portion of the study $(n=677)$. Similarly, internal reliability for each fraction arithmetic operation was high. Cronbach's alpha was $0.85,0.86,0.93$, and 0.94 for addition, subtraction, multiplication, and division, respectively.

\section{Fraction Knowledge Profiles}

In this subsection, I describe the process of obtaining adults' fraction knowledge profiles through cluster analysis and the interpretation of those results.

Cluster analysis of standardized residualized scores. To assess the optimal number of participants' fraction knowledge profiles for adults, I residualized participants' conceptual and procedural percentage scores against each other before performing a cluster analysis (Hallett et al., 2010; 2012). This method creates measures of conceptual and procedural knowledge that separates out the shared variance attributed to each of these two types of knowledge. The procedural score for each participant was regressed against their conceptual score and recorded for each participant as the residualized procedural score. The same procedure was used to create a residualized conceptual score. Next, I performed a cluster analysis on 
participants' residualized conceptual and procedural scores using Ward's clustering method to obtain estimates of centroids for clusters (Ward, 1963). Centroids obtained by this method were then subjected to a k-means cluster analysis.

Three criteria were used to determine the optimal number of clusters (see Hallett et al., 2012). The first criterion for selecting the optimal solution is that solutions should have high-within cluster variances and low-between cluster variances, resulting in large $\mathrm{c}(\mathrm{g})$ values. The $\mathrm{c}(\mathrm{g})$ statistic is an index that calculates the ratio of the between-cluster variance to within-cluster variance for each solution (Calinski \& Harabasz, 1974). The second criterion is that optimum solutions should not have splinter clusters. Splinter clusters can be thought of as outliers. Splinter clusters typically have large variances for subjects within the splinter cluster and comparatively smaller between-cluster variances, inflating the $\mathrm{c}(\mathrm{g})$ value. The third criterion for choosing the best solution is that the two-cluster solution needs to be preferable to a single-cluster solution for profiling the sample. The $\mathrm{L}(\mathrm{m})$ statistic recommended by Duda and Hart (1973) was used to evaluate this criterion. To calculate $\mathrm{L}(\mathrm{m})$, the sum of variances from each data point to its cluster centroid in the two-cluster solution is compared with the sum of variance between data points and the centroid for the single-cluster solution. A statistically significant $\mathrm{L}(\mathrm{m})$ value provides evidence that that the two-cluster solution is a better solution than the single-cluster solution.

To determine the optimal solution for the three criteria, I first obtained the optimal number of clusters by calculating the $\mathrm{c}(\mathrm{g})$ statistic for each solution when the number of clusters ranged from 2 to 13 (see Table 3.7). Solutions of 14 or more clusters are not presented as the fourteen-cluster solution had splinter clusters consisting of fewer than five participants. Based on the above two criteria, the Ward 
Method identifies the two-cluster solution as the solution with the highest $\mathrm{c}(\mathrm{g})$ value. To assess the third criterion, I computed the $\mathrm{L}(\mathrm{m})$ statistic for the sample. $\mathrm{L}(\mathrm{m})$ was statistically significant, $\mathrm{L}(\mathrm{m})=6.95, p<.001$, suggesting that the two-cluster solution was a better solution than a single-cluster solution. In summary, cluster analysis shows that adults could be optimally clustered into two groups based on their residualized procedural and conceptual fraction knowledge scores. The number of people in each group was similar, Group 1: $n=346$, Group 2: $\mathrm{n}=374$. 
Table 3.7: $c(g)$ values for k-means cluster solutions using standardized residual scores for conceptual and procedural knowledge.

\begin{tabular}{lc}
\hline Number of Clusters & C(g) value using Ward's Method as starting point \\
\hline 2 & $\mathbf{7 7 1 . 7 ^ { \mathbf { a } }}$ \\
3 & 718.3 \\
4 & 677.4 \\
5 & 741.9 \\
6 & 725.0 \\
7 & 730.0 \\
8 & 740.2 \\
9 & 699.1 \\
10 & 760.1 \\
11 & 750.0 \\
12 & 735.6 \\
13 & 739.4 \\
\hline
\end{tabular}

Figure 3.1 presents a scatterplot of the participants' residualized conceptual and procedural scores. There was a strong correlation between participants' residualized conceptual and procedural scores, $r(719)=-.65, p<.001$. One group showed higher than expected residual procedural scores and lower than expected conceptual scores. Conversely, the other group showed higher than expected residual conceptual scores and lower than expected procedural scores. I labelled these groups as procedurally stronger and conceptually stronger to reflect each groups' relative strengths on procedures and concepts respectively. Figure 3.2 presents the mean residualized procedural and conceptual scores for each group. Residualized 
conceptual and procedural scores for adults show the same pattern as the scores observed in 8th grade students by Hallett et al (2012).

Figure 3.1: Scatterplot for participants' residualized conceptual and procedural scores. Group 1 was the procedurally-stronger group $(n=346)$ and Group 2 was the conceptually-stronger group $(n=374)$.

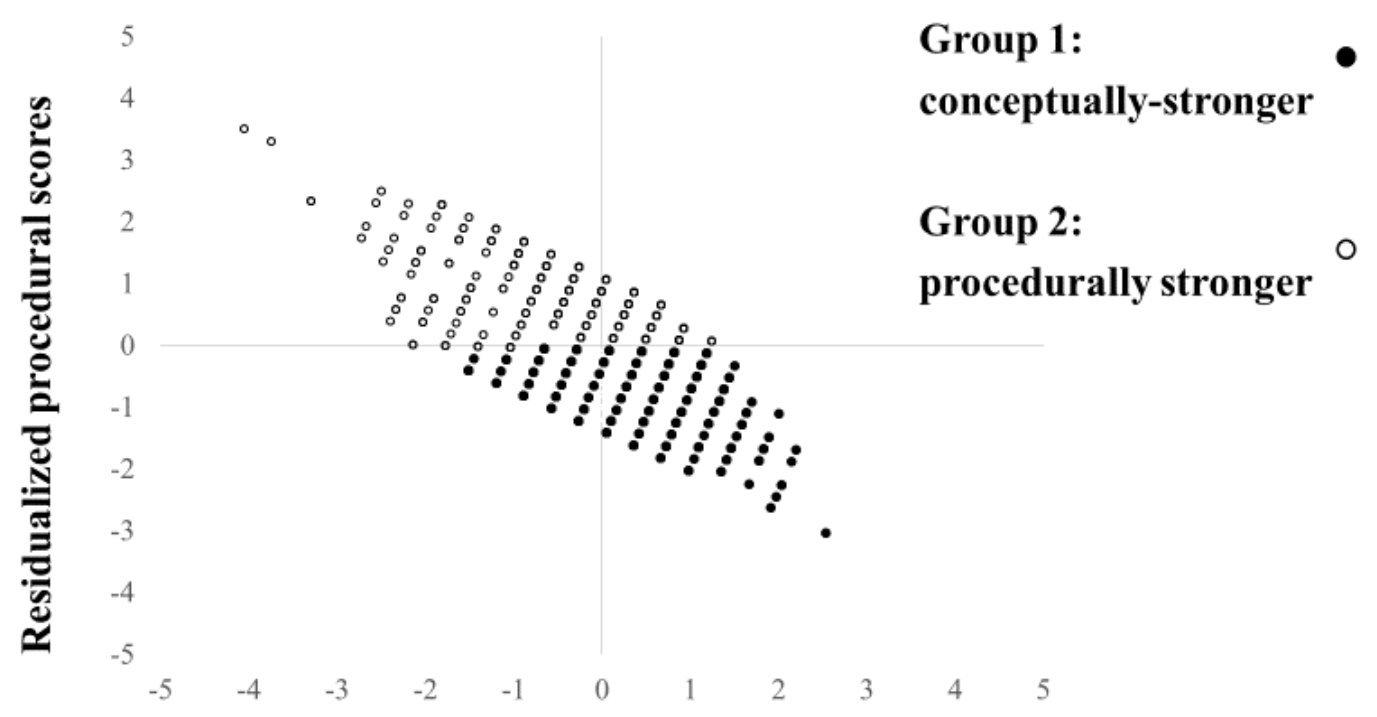

\section{Residualized conceptual scores}

Figure 3.2: Average means for standardized residualized conceptual and procedural scores obtained by members of Group 1: the procedurally-stronger group $(n=346)$ and Group 2: the conceptually-stronger group $(n=374)$. Error bars represent the standard error of the mean.

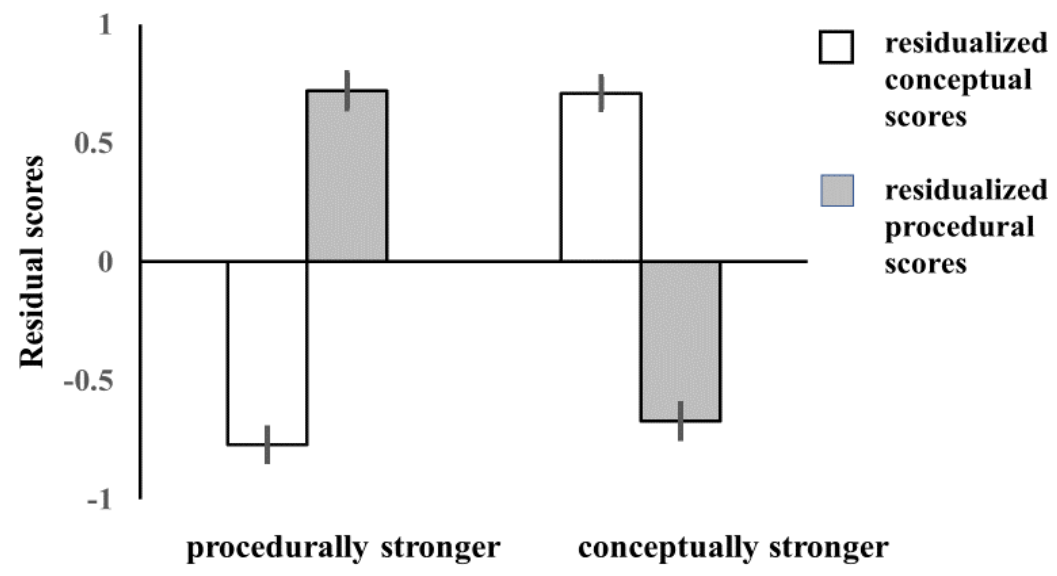


To assess the differences in fraction knowledge and arithmetic knowledge between the two groups of solvers, I compared test scores obtained on each FKA assessment between both groups. Figure 3.3 presents conceptual and procedural FKA scores for each group. Scores are presented in percentages to allow for suitable comparisons across both fraction measures.

Figure 3.3: Mean conceptual and procedural scores for the procedurally-stronger group $(n=346)$ and conceptually-stronger group $(n=374)$. Error bars are 95\% CI calculated according to Jarmasz and Hollands (2009).

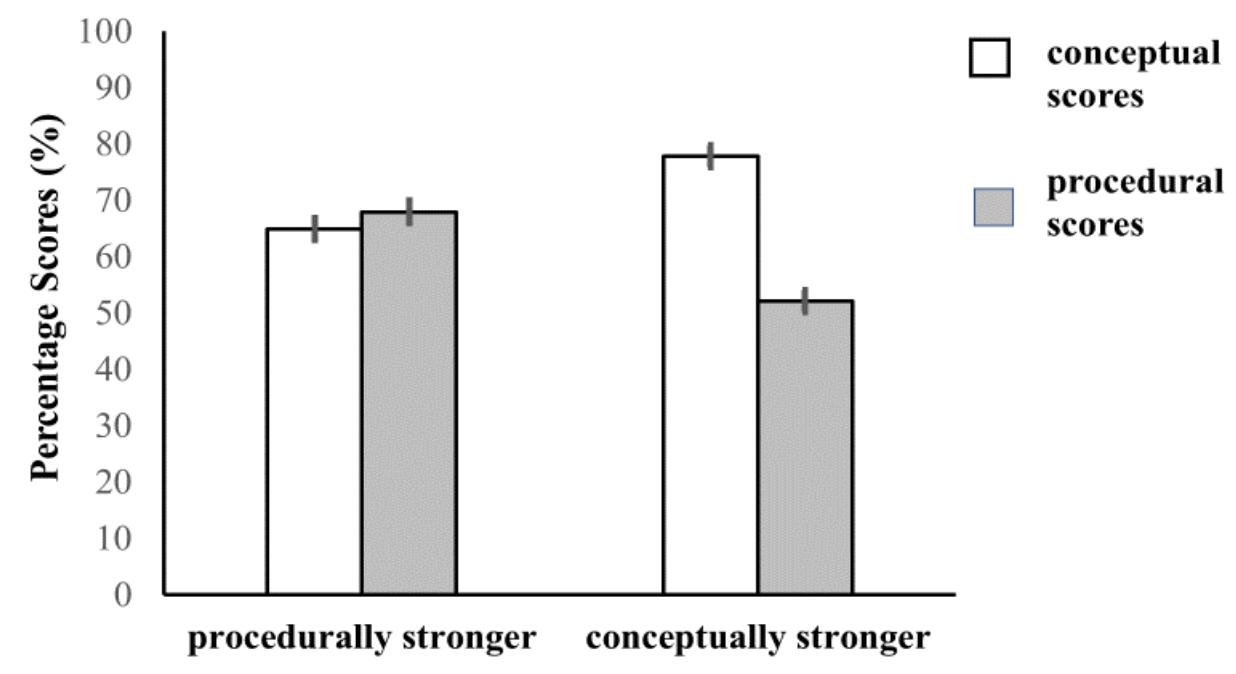

Figure 3.3 shows that the procedurally-stronger group had similar conceptual and procedural scores. This group also had significantly higher procedural scores than the conceptually-stronger group (consistent with the results of the residualizing procedure). In contrast, the conceptually-stronger group had significantly higher conceptual than procedural scores and also significantly higher conceptual scores then the procedurally-stronger group (note, the confidence intervals were used to interpret these patterns, as shown in the figure). Furthermore, Figure 3.3 also suggests that overall conceptual scores were higher than procedural scores across all participants, indicating that the procedural section was more difficult that the conceptual section of the FKA. (Personal communication with Dr. Hallett). 
To summarize, cluster analysis on residual scores revealed adults could be profiled into two fraction knowledge groups - a procedurally-stronger group and a conceptually-stronger group. Members of the procedurally-stronger group performed relatively better on procedures and relatively worse on concepts. They also performed better on procedural problems than the conceptually-stronger group and performed equally well with both fraction concepts and procedures. Members of the conceptually-stronger group performed relatively better on concepts and relatively worse on procedures. They also performed better on conceptual items than the procedurally-stronger group and performed better when working with concepts than procedures.

Basic arithmetic test scores for fraction knowledge profiles. In this section, I compare each profile group's performance basic arithmetic scores (CFT and BMT).

BMT and CFT scores. Table 3.8 shows mean scores and standard deviations for conceptually- and procedurally-stronger solvers on CFT and BMT problems and the results of independent sample t-tests between scores obtained by each group of solvers. As shown in Table 3.8, procedurally- and conceptually-stronger solvers showed equal levels of performance on all subscales of the CFA and on small singledigit multiplication problems. However, procedurally-stronger solvers performed better than conceptually-stronger solvers on large single-digit multiplication problems. Overall, solvers in both groups showed comparable levels of performance on multi-digit arithmetic problems, with a small advantage for procedurally-stronger solvers on single-digit multiplication with products larger than 25 .

MBIQ scores. A summary of participants' responses on MBIQ scores that assessed mathematics behaviours and rated self-efficacy are presented in Appendices E and F. Overall, procedurally-stronger solvers rated their levels of mathematics and 
fraction skills higher than did conceptually-stronger solvers. Otherwise, there appeared to be no marked differences between groups. Thus, conceptually- and procedurally-stronger solvers did not differ in their reported behaviours when solving arithmetic problems. 
Table 3.8: Means and standard deviations of procedurally-and conceptually-stronger solvers on the CFT and BMY total scores.

\begin{tabular}{|c|c|c|c|c|c|c|c|c|c|c|}
\hline \multirow[t]{2}{*}{ Measure } & \multicolumn{3}{|c|}{ procedurally-stronger } & \multicolumn{3}{|c|}{ conceptually-stronger } & \multirow[b]{2}{*}{$d f$} & \multirow[b]{2}{*}{$t$} & \multirow[b]{2}{*}{$p$} & \multirow[b]{2}{*}{ Cohen's d } \\
\hline & $M$ & $S D$ & $n$ & $M$ & $S D$ & $n$ & & & & \\
\hline BMT (large) & 14.5 & 8.0 & 344 & 12.9 & 7.4 & 370 & 712 & $2.68^{*}$ & $<.01$ & 0.21 \\
\hline BMT (small) & 27.7 & 10.7 & 345 & 26.8 & 10.3 & 372 & 715 & 1.10 & 0.27 & 0.09 \\
\hline CFT (add) & 9.4 & 5.1 & 345 & 9.4 & 4.0 & 374 & 717 & 0.15 & 0.88 & $<.001$ \\
\hline CFT (subtract) & 8.7 & 4.1 & 343 & 8.7 & 4.4 & 373 & 714 & 0.08 & 0.94 & $<.001$ \\
\hline CFT (multiply) & 6.3 & 4.2 & 337 & 6.0 & 4.3 & 351 & 686 & 0.99 & 0.32 & 0.07 \\
\hline
\end{tabular}




\section{Discussion}

The primary goal of this study was to identify adult fraction profiles based on their conceptual and procedural knowledge of fractions. Profiles were subsequently used to determine membership for experimental conditions in Experiments 2 and 3. However, Study 1 was also designed to test whether findings with middle-school students extended to adults (Hallett et al., 2012) and to identify each group's performance on their understanding of fraction principles and their ability to perform basic mental arithmetic.

\section{What Fraction Knowledge Profiles were Observed for Adults?}

Results from the cluster analysis of residualized fraction knowledge scores showed that adults could be profiled into two groups based on their strengths on procedural and conceptual knowledge - a procedurally-stronger group and a conceptually-stronger group. The procedurally-stronger group performed better than expected on procedural problems, given their performance on conceptual problems. The conceptually-stronger group performed better than expected on conceptual problems, given their performance on procedural problems. Residualized conceptual and procedural fraction assessment scores for the two groups showed similar patterns to fraction profiles of $8^{\text {th }}$ grade students reported by Hallett et al. (2012).

\section{Characteristics of Each Fraction Knowledge Group}

The procedurally-stronger group performed slightly better on large basic multiplication problems and rated themselves as more skilled at arithmetic and fraction problem solving. It is striking to note that procedurally-stronger solvers also performed better than conceptually-stronger solvers on the procedural FKA. However, procedurally strong solvers performed worse than conceptually-stronger solvers on knowledge of fraction concepts. Furthermore, the groups did not differ in 
their performance on the simplest multiplication problems or on multi-digit arithmetic problems. I speculate that solvers' self evaluations of their performance on basic and fraction arithmetic is related to their ability to retrieve and/or compute arithmetic facts and perform fraction related procedures. In Chapter 4, I analyzed solvers' responses and inferred their strategies as they solved fraction arithmetic problems to assess whether fraction knowledge that can be uniquely attributed to concepts or procedures plays a role in solvers' strategy selections. 


\section{CHAPTER 4: FRACTION ARITHMETIC PROBLEMS (FARRA)}

In this chapter, I analyzed fraction arithmetic (FARRA) problems from Study 1. These problems had previously been used in studies with middle-school students and to assess learning in FARRA (Braithwaite et al., 2017; Siegler \& Pyke, 2013; Siegler et al., 2011). In Chapter 3, adults were profiled into a procedurally-stronger group and a conceptually-stronger group based on their performance on the Fraction Knowledge Assessment. The procedurally-stronger group performed better than the conceptually-stronger group on fraction problems that had been identified as assessing fraction arithmetic in middle-school students. This chapter addresses the general question: What factors cue adults to select strategies as they perform fraction arithmetic? However, the general question can be divided into three parts.

(1) What are the factors that influence adults to select correct strategies?

(2) What incorrect strategies do adults apply when they generate systematic errors?

(3) What are the factors that influence adults' selection of incorrect strategies? Solvers need to be able to select and apply the correct strategy for each operation to obtain correct answers. Accuracy rates are therefore a measure of correct strategy selection in fraction arithmetic. Previous findings with middle-school students showed that accuracy rates were highest for fraction addition and subtraction and lowest for fraction division (Braithwaite et al., 2017; Siegler \& Pyke, 2013). Siegler et al. (2011) analyzed responses made by middle-school students to fraction arithmetic problems and identified two types of strategy errors. For fraction addition and subtraction, the most common strategy error was to treat the numerators and denominators of each fraction as if they were independent and apply the indicated arithmetic operation to each component. For example, when presented with the 
problem $3 / 5+1 / 2$, solvers added the numerators $3+1=4$ and the denominators $5+2=7$ to generate the solution $4 / 7$. Similarly, when presented with the subtraction problem $3 / 5-1 / 2$, solvers subtracted the value of the numerator in the second fraction from the numerator of the first fraction 3-1=2; and the value of the denominator in the second fraction from the denominator of the first fraction $5-2=3$ to generate the solution $2 / 3$. Siegler and his colleagues called these errors "independent whole number errors" because solvers treated each number in the numerator and denominator as separate whole numbers rather than treating the numbers as components of a fraction.

For fraction multiplication and division, the most common error involved selection and application of an incorrect procedure, specifically, maintaining the denominator while applying the required operation to the numerator (Siegler et al., 2011). For example, when presented with the problem $3 / 5 \times 2 / 5$, solvers multiplied the numerators $3 \times 2=6$ and maintained the common denominator 5 to generate the solution $6 / 5$. Similarly, for the division problem, $3 / 5 \div 2 / 5$, solvers divided the fraction of the second numerator by the first numerator $3 \div 2=1.5$ and maintained the common denominator " 5 " to generate the solution " $1.5 / 5$ ". These strategy errors are called wrong fraction operation errors because they involved a step for the procedure normally required to perform fraction addition and subtraction. For middle-school students, independent whole number errors were most frequent for fraction addition and subtraction. In contrast, wrong fraction operation errors were most frequent for fraction multiplication and division. (See Table 4 in Schneider et al., 2011, p287). The characteristics of the problem - specifically, the required operation and the relation between denominators cued the strategy selected by these students.

The incorrect strategies identified by Siegler et al. (2011) and the conditions under which they are observed have been replicated in other studies with middle- 
school students (Braithwaite \& Siegler, 2018; Siegler \& Pyke, 2013) and have been simulated using a computer model (Braithwaite et al., 2017). The frequencies of strategy use for $8^{\text {th }}$ grade students are taken from Siegler et al (2013) and are presented in Table 4.1. Note that when dividing fractions, $8^{\text {th }}$ grade students, $23 \%$ of $8^{\text {th }}$ grade divided both numerators and denominators (i.e., $3 / 5 \div 2 / 5=1.5 / 1$ ). Siegler and his colleagues coded this strategy error as independent whole number errors, whereas multiplying numerators and denominators was the correct procedure for multiplication.

Table 4.1. Percent use of fraction arithmetic strategies in $8^{\text {th }}$ grade students

\begin{tabular}{lccccc}
\hline Operation & Denominators & Correct & $\begin{array}{c}\text { Independent } \\
\text { whole } \\
\text { number }\end{array}$ & $\begin{array}{c}\text { Wrong } \\
\text { fraction } \\
\text { operation }\end{array}$ & $\begin{array}{c}\text { None/ } \\
\text { unknown }\end{array}$ \\
\hline Addition & equal & 75 & 25 & 0 & 0 \\
Addition & unequal & 58 & 38 & 0 & 4 \\
Subtraction & equal & 71 & 25 & 4 & 0 \\
Subtraction & unequal & 58 & 38 & 0 & 0 \\
Multiplication & equal & 71 & - & 25 & 4 \\
Multiplication & unequal & 79 & - & 17 & 0 \\
Division & equal & 46 & 25 & 17 & 8 \\
Division & unequal & 50 & 21 & 25 & 4 \\
\hline
\end{tabular}

Cluster analysis of adults' fraction knowledge scores revealed that adults could be profiled as conceptually-stronger or procedurally-stronger solvers. These profiles and their relative strengths in conceptual and procedural fraction knowledge were also identified in $8^{\text {th }}$ grade students in Hallett et al. (2012). Thus, adults' accuracy patterns with fraction arithmetic and their errors were also expected to follow similar patterns to those observed in $8^{\text {th }}$ grade students.

To summarize, adults' performance and errors should show similar patterns to 
$8^{\text {th }}$ grade learners. I hypothesize that:

(1) Accuracy will be highest for fraction addition and subtraction and lowest for fraction division.

(2) For fraction addition and subtraction problems, the most frequent strategy error will be the independent whole number strategy. It will be frequently observed for different denominator than same denominator problems.

(3) For fraction multiplication and division problems, the most frequent strategy error will be the wrong fraction operation strategy. It will be more frequently observed for same- than different-denominator problems.

The two groups of solvers identified in Chapter 3 - conceptually-stronger and procedurally-stronger solvers - differed mainly in their relative understanding of conceptual and procedural knowledge and allowed me to test a further prediction of how solvers' strengths and weaknesses in each type of knowledge might have an impact on how they solved fraction arithmetic problems. Specifically, I hypothesized that procedurally-stronger solvers were able to better retrieve, select, and apply correct procedures for the required operation than conceptually-stronger solvers. As such, I tested two more hypotheses that highlighted the relations between solver knowledge and their performance and strategy selection:

(4) Procedurally-stronger solvers will be more accurate than conceptuallystronger solvers.

(5) Procedurally-stronger solvers will select the independent-whole number and wrong fraction operation strategies less frequently than conceptuallystronger solvers. 


\section{Results}

\section{Accuracy}

Forty-three $(6.0 \%)$ of the participants provided a response for at least one problem on the FARRA assessment, but did not provide any correct response (i.e., received a score of 0 ). Twenty-two of these participants were procedurally-stronger solvers; 21 were conceptually-stronger solvers. Data for these participants were excluded from the analysis. Of the remaining 677 participants (324 procedurallystronger solvers, 353 conceptually-stronger solvers), there were 406 women (60.0\%) and 271 men (40.0\%). Problems were scored as correct if the correct solution was provided in either fractions or decimals. Participants' total scores were expressed as percentages calculated from the number of correct responses for the eight groups of problems defined by fraction operation, 4 (addition, subtraction, multiplication, division), and the relation between denominators, 2 (same denominator, different denominator). There were three items in each of the eight groups. (See Table 3.4).

Accuracy scores (percentage correct) were analyzed in a 2(group: procedurally-stronger vs. conceptually-stronger) by 2(denominator: same vs. different) by 4 (operation: addition, subtraction, multiplication, division) with repeated measures on the last two factors. Post-hoc comparisons were tested using Tukey HSD at .05 level of significance.

Solvers were more accurate on problems that shared a common denominator compared to those where denominators differed $(75.4 \%$ vs. $67.9 \%), F(1,675)=$ $144.9, p<.001, \eta^{2}=.18$. There was also a significant main effect of operation, $F$ $(3,673)=70.0, p<.001, \eta^{2}=.24$. Solvers were more accurate on addition $(76.7 \%)$, subtraction (77.7\%), and multiplication (73.7\%) than division problems (58.1\%), ps $<$ .01 whereas accuracy did not differ for addition, subtraction and multiplication 
problems. This pattern of results was observed in middle-school students (Siegler et al., 2011; Siegler \& Pyke, 2013). These main effects were qualified by a significant interaction between operation and denominator, $F(3,673)=74.7, p<.001, \mathrm{\eta}^{2}=.25$, as shown in Figure 4.1. Accuracy for same denominator problems was better than for different denominator problems when solvers were required to add or subtract fractions. However, accuracy for same and different denominator problems did not differ between fraction multiplication and division problems.

Figure 4.1. Percentage scores obtained by procedurally-and conceptually-stronger solvers by operation (addition, subtraction, multiplication and division) and whether each problem had the same or different denominators. Error bars are 95\% CI calculated according to Jarmasz \& Hollands (2009).
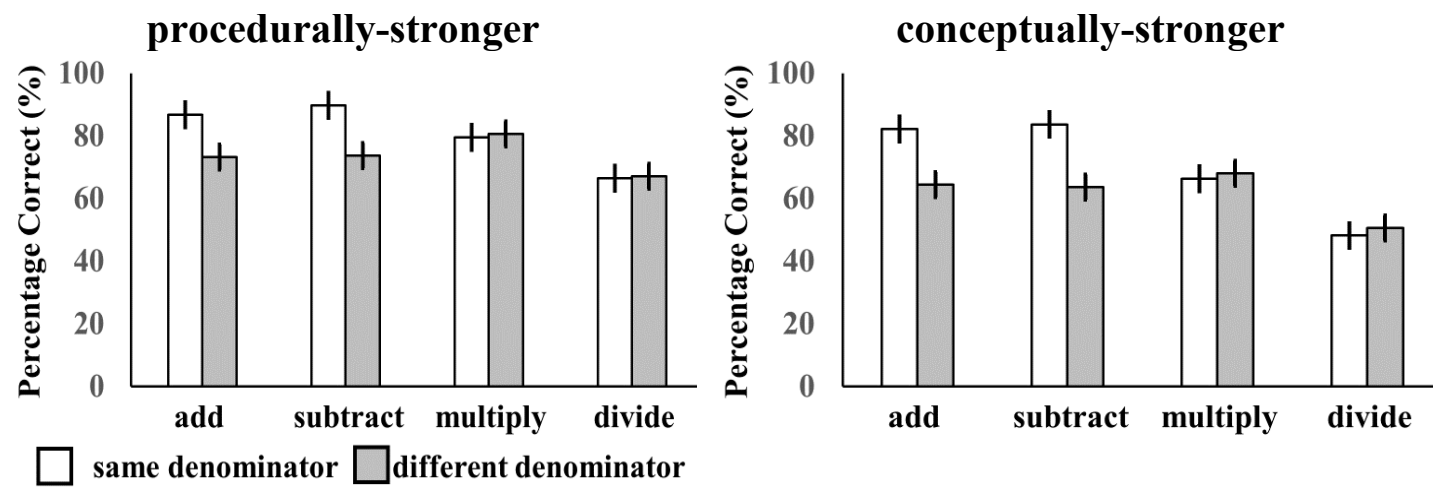

As expected, procedurally-stronger solvers solved fraction arithmetic problems more accurately than conceptually-stronger solvers (77.2\% vs. $65.9 \%), F$ $(1,675)=27.5, p<.001, \eta^{2}=.04$. As shown in the figure, the pattern of performance across operation and denominator relation was identical for the two groups. No interactions between group and the other variables were significant.

In general, adults made the most errors on division problems compared to the other three operations, suggesting that the division algorithm was the least familiar or worst remembered. Adults also made more errors on different denominator problems 
than same denominator problems on addition and subtraction problems whereas there was no difference between multiplication and division problems. This pattern of data suggests that the requirement to find common denominators on addition and subtraction problems is a source of difficulty for these adults. To address the other hypotheses posed in the introduction, solvers' responses were analyzed. What errors do solvers generate when they perform fraction arithmetic? Specifically, what kinds of errors are observed when solvers add or subtract same denominator problems and what is the source of these errors that makes these problems difficult? I will now examine solvers' responses to assess their most frequent errors and infer the strategies that were used to generate solvers' errors.

\section{Most Frequent Responses}

The most frequently generated responses for each problem were analyzed to infer strategy selection. The ten most frequent responses for each problem were noted and tabulated. These responses were used to infer the strategies used to solve each type of fraction arithmetic problem. When it was not possible to infer the strategy that the participant used, I reported the actual response. The frequency of the most common responses and their percentages of all participants for each fraction arithmetic operation are presented in Appendix G.

For each strategy, I first describe participants' common responses and the inferred strategy for each response. I then provide a quantitative analysis and comparison of the frequency of responses across solver group, operation and denominator relationship. Percentage frequencies were calculated based on the number of times that a strategy was selected by each solver for problems of each operation and denominator type. Post-hoc comparisons were performed using Tukey's HSD at .05 level of significance. 
In the following two sections, I will provide a qualitative analysis of solvers' responses and the types of errors to draw inferences about the strategies that were used to solve each type of problem. First, I will discuss correct strategies other than those generated through FARRA procedures. I will then analyze common error responses provided by solvers.

Correct strategies. Most solvers provided fraction answers to the majority of problems that were the result of correctly applying the FARRA algorithms. However, solvers used other correct strategies on some problems. Specifically, solvers sometimes converted fractions to decimals and they sometimes reduced fractions before applying the required operation. These strategies resulted in correct answers that were different from those that would be produced by FARRA and so they are described separately.

Conversion to decimals. Some solvers converted a fraction into its equivalent decimal format, either during calculation or when they produced a solution. For example, when a decimal answer was given for the problem, $3 / 5+1 / 2$, solvers may have converted $3 / 5$ into the decimal equivalent 0.6 and $1 / 2$ into the decimal equivalent 0.5 , then added the decimals to obtain the answer 1.1. Alternatively, they may have applied the correct strategy to obtain the corresponding solution $11 / 10$, then converted the correct solution into its decimal equivalent 1.1. It is not possible to determine when the decimal conversion was done in the course of problem solving

Percentages of the frequency of fraction conversion into decimals were calculated based the total number of correct decimal answers provided by each participant for problems grouped by operation and denominator relationship. Figure 4.2 presents the average percentage frequencies of correct decimal solutions by solver group, operation and denominator relationship as a proportion of total responses 
categorized by operation and denominator relationship. Incorrect decimal responses were not analyzed, as it was not possible to infer the steps that solvers used to generate these responses. A $2 \times 4 \times 2$ ANOVA with solver group as the betweensubjects variable and operation and denominator as within-subjects variables were performed on average frequencies.

Figure 4.2. Average percentage of correct trials on which participants converted fractions into decimals by operation and denominator relationship. Error bars are 95\% CI calculated according to Jarmasz and Hollands (2009).
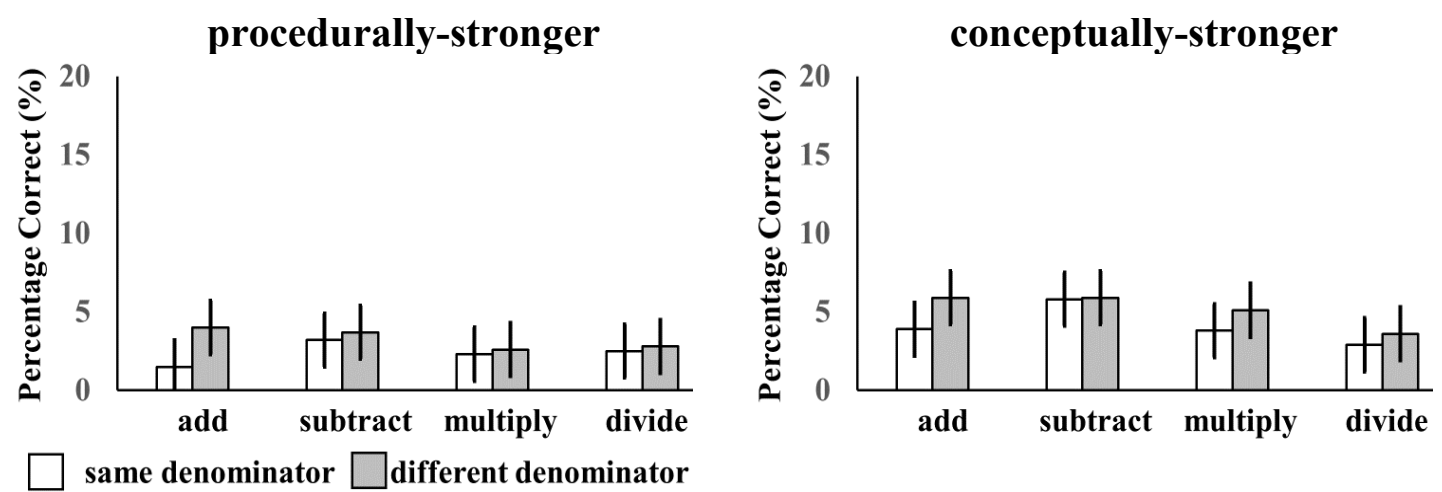

There was no significant difference in the percentage of correct trials on which conceptually- versus procedurally-stronger solvers converted fractions into decimals $(2.8 \%$ vs. $4.6 \%), F(1,675)=3.14, p=.07$. Solvers converted fractions into decimals more frequently for different denominator than same denominator problems $(4.2 \% \mathrm{vs}$. $3.2 \%), F(1,675)=136.53, p<.001, \eta^{2}=.17$. A significant difference was also observed across operations, $F(3,673)=3.67, p=.01, \eta^{2}=.02$ : Solvers converted fractions to decimals more frequently for subtraction $(4.7 \%)$ than multiplication $(3.4 \%)$ or division $(2.9 \%), p s<.05$. However, decimal conversion was not applied more frequently for addition $(3.8 \%)$ than the other three operations. An interaction was also observed between operation and denominator, $F(3,673)=4.57, p<.005, \eta^{2}$ $=.02$. When adding fractions, solvers converted fractions to decimals more frequently 
for different denominator than same denominator problems (4.9\% vs. $2.7 \%)$. For the other three operations, there was no preference to convert fractions across same and different denominator problems.

Overall, solvers were more likely to convert fractions into decimals for subtraction and for different denominator addition problems over the other kinds of problems. Solvers preferential use of decimals for some problems over others may have occurred because adding or subtracting decimals would be easier than multiplying or dividing them. For example, $4 / 5+3 / 5$ could be converted to $0.8+0.6$ to provide the answer 1.4 if the decimal forms of these fractions was easily available to solvers. In contrast, multiplying $0.6 \times 0.8$ may have been more difficult than multiplying $4 \times 3$ and thus less likely to be an appealing strategy.

Reduction. There were four FARRA problems, one multiplication and three division, where solutions could be expressed as either reducible or non-reducible fractions. Clearly, this problem set was not designed to provide opportunities for reduction, and so the data are only suggestive and may not represent a full range of possible solutions. The one multiplication problem which was reducible was $2 / 3 \times$ 3/5; this problem might have either cued reduction before calculation (if solvers noticed the $3 \mathrm{~s}$ in both denominator and numerator positions), or could have been reduced after calculation (i.e., $6 / 15$ to $2 / 5$ ). For example, when presented with the problem $2 / 3 \times 3 / 5$, solvers may have multiplied the numerators $2 \times 3$ and the denominators $3 \times 5$ to obtain the solution. $6 / 15$. However, the reduced form of this fraction, $2 / 5$, was also a common solution to the problem. About twice as many solvers provided the response $6 / 15$ compared to the reduced fraction $2 / 5$. The response $2 / 5$ was interesting in this context because it required applying an additional step. Because only the answer was required, these two possibilities are 
indistinguishable.

Evidence of reduction was also observed for the three same-denominator division problems that had common factors. None of the different-denominator problems, in contrast, were reducible, because they did not share a common factor. For the same-denominator division problems, solvers were more likely to answer with the fully reduced fraction than with the unreduced fraction. For example, when presented with the problem $3 / 5 \div 2 / 5$, they answered $3 / 2$ rather than $15 / 10$. This response suggests that, in the process of solving the problem, solvers identified the common factor in the numerator and denominator 5 and simplified both numerator and denominator by a denominator of 5 resulting in the reduced solution $3 / 2$. For the different denominator problems, none of the items could be reduced. Figure 4.3 presents the average percentage frequencies across these problems when solvers provided correct reduced solutions by group and operation.

Figure 4.3. Average frequency of selections (in \%) for correct reduced solutions by operation and group. Error bars are 95\% CI calculated according to Jarmasz and Hollands (2009).

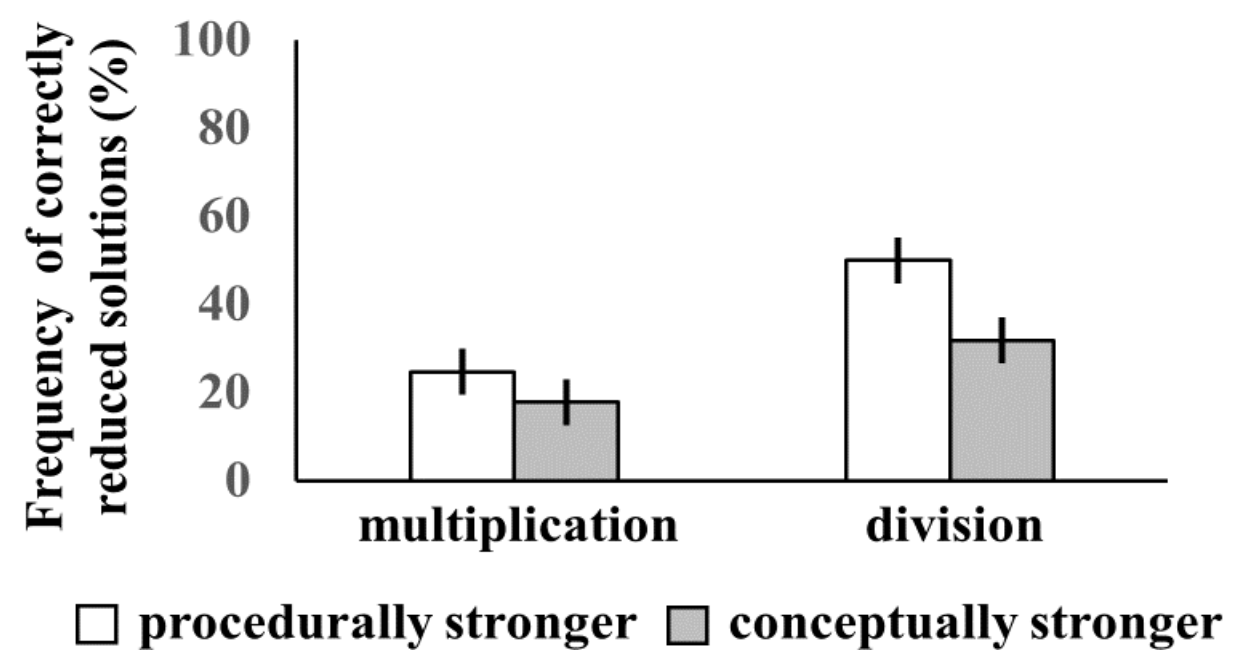

A $2 \times 2$ ANOVA with solver group as a between-subjects factor and operation as a within-subjects factor revealed a main effect of operation. Solvers provided 
correct reduced solutions more frequently for division than multiplication (40.9\% vs. $21.3 \%), F(1,675)=136.64 p<.001, \eta^{2}=.17$. Procedurally-stronger solvers provided correctly reduced solutions more frequently than conceptually-stronger solvers $(37.3 \%$ vs. $24.8 \%), F(1,675)=21.9, p<.001, \eta^{2}=.03$, but this main effect was qualified by the interaction between group and operation, $F(1,675)=11.37, p<.005$, $\eta^{2}=.02$. When dividing, procedurally-stronger solvers used reduction to obtain correct solutions more frequently than conceptually-stronger solvers $(50.0 \% \mathrm{vs}$. $31.8 \%$ ). When multiplying fractions, procedurally-stronger solvers also reduction more frequently than conceptually-stronger solvers ( $24.7 \%$ vs. $17.8 \%)$, but the difference was smaller. These results suggest that at least for the FARRA problems, division was more likely to cue selection of the reduction algorithm than multiplication, and that reduction was selected more frequently for solvers with stronger procedural than conceptual knowledge.

When dividing fractions, procedurally-stronger solvers were cued to use reduction most often on same-denominator division problems. However, when multiplying fractions, both solver groups used reduction with equal frequency. These results suggest that individual differences and features of problems may both be important factors in solvers' selection of reduction. However, because this item set did not provide equal opportunities for use of reduction across operations or problem types, these conclusions should be interpreted cautiously.

Incorrect strategies. Solvers generated a variety of incorrect responses. As with middle-school students, solvers also systematically selected incorrect strategies that led to an incorrect response. These incorrect strategies are the independent whole number strategy, the wrong fraction operation strategy (Siegler et al., 2011) and the wrong inversion strategy (see below). These strategies are described because the 
selected strategies were selected by more than $5 \%$ of all participants, and as such were assumed to be a product of solvers systematically selecting wrong strategies.

Independent whole number strategy. The independent whole number strategy was the most frequently observed incorrect strategy for both addition and subtraction. Solvers added or subtracted the numerators and denominators rather than determining a common denominator. For example, on the addition problem $3 / 5+1 / 5$, solvers added the numerators $3+1$ and the denominators $5+5$ to obtain the answer $4 / 10$. Similarly, for the subtraction problem 3/5-1/4, solvers subtracted the numerators $3-1$ and the denominators 5-4 to obtain the answer 2/1. The strategy was seldom used when dividing fractions and was the correct strategy when multiplying fractions.

The percentage use of the independent whole number strategy was analyzed in a 2 (operation: addition, subtraction) $\times 2$ (solver group: conceptual, procedural) $\times 2$ (denominator: same, different) mixed ANOVA. Independent whole number errors were more frequent for conceptually-stronger than the procedurally-stronger group $(6.6 \%$ vs. $3.1 \%), F(1,675)=68.07, p<.001, \eta^{2}=.09$ (see Figure 4.4$)$. This pattern is consistent with the way in which these groups were selected: Because the conceptually-stronger group was procedurally weaker it is not surprising that they were more likely to use an incorrect procedure.

The independent whole number strategy was more frequently used on addition than subtraction $(6.3 \%$ vs. $3.4 \%), F(1,675)=26.65, p<.001, \eta^{2}=.04$, and for different-denominator than same-denominator problems $(6.6 \%$ vs. $3.1 \%), F(1,675)=$ $44.28, p<.001, \eta^{2}=.06$. Furthermore, there was a significant interaction between denominator and operation, $F(1,675)=27.02, p<.001, \eta^{2}=.04$, as shown in Figure 4.4 for each group. Post-hoc comparisons revealed that for subtraction, the strategy was less frequently selected for different- than same-denominator problems $(0.4 \% \mathrm{vs}$. 
$6.4 \%), p<.001$ whereas for addition, frequencies did not vary between different denominator and same denominator problems (5.8\% vs. $6.8 \%)$.

Figure 4.4. Average frequency of use of the independent whole number strategy by group. Error bars are 95\% CI calculated according to Jarmasz and Hollands (2009).
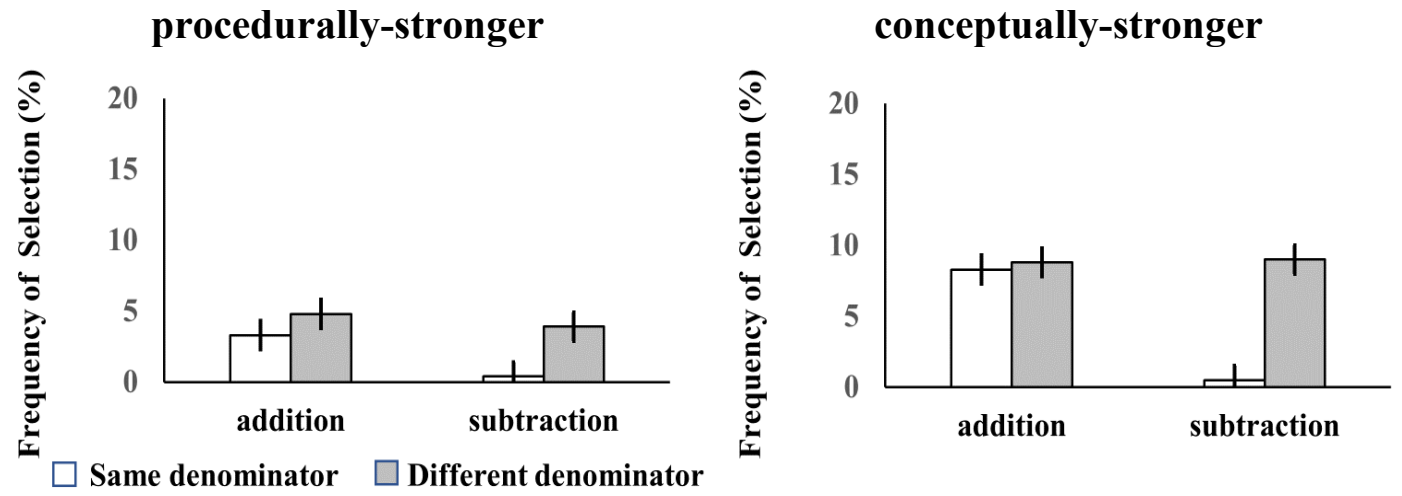

In general, the independent whole number strategy was more frequently selected by conceptually- than procedurally-stronger solvers, However, it is clear from Figure 4.4 that the pattern with which this strategy was applied was similar for the two groups. Solvers used this strategy selectively on the three differentdenominator problems. For the two of these problems, 3/5-1/2 and 3/5-1/4, both numerators and denominators of the first fraction were larger than the numerators and denominators of the second fraction. For these two problems, the most frequent error involved application of the independent whole number strategy, resulting in the solutions $2 / 3$ and $2 / 1$. Application of the independent whole number strategy to the problem $2 / 3-3 / 5$ would have generated the answer $-1 /-2$. Instead, the most frequent error generated by adults for this problem was the response 1/2. Presumably, solvers selected the independent whole number strategy, then further used their knowledge that the division of two negative numbers was a positive number to produce a positive fraction.

For subtraction problems with the same denominators, the application of the 
independent whole number strategy would have resulted in a solution with a denominator of 0 (i.e., 2-2=0). Responses by a few solvers indicated that they used the independent whole number strategy (e.g., 3/5-2/5=1/0). Nevertheless, this strategy was less frequently observed than on the same-denominator problems, presumably because these adults knew that dividing by 0 is not possible. Instead, the most frequent incorrect strategy on same denominator problems was to subtract the second numerator from the first numerator and divide the denominators by themselves. Applying this strategy to all three same denominator subtraction problems: $3 / 5-1 / 5$; $3 / 5-2 / 5$ and $4 / 5-3 / 5$ would have produced the corresponding responses $2 / 1 ; 1 / 1$ and $1 / 1(0.4 \%$ of all problems $)$ or the reduced responses $2 ; 1$ and $1(3.4 \%$ of all problems). These responses were the most frequent incorrect responses produced by solvers for same denominator fraction subtraction. This strategy seemed to be a special case of the independent whole number strategy that was modified to avoid having a denominator of 0 .

In summary, the independent whole number strategy was selected by solvers when they were adding fractions and when subtracting different-denominator fractions. On same-denominator problems, the most common error strategy was a modified version that avoided a zero in the denominator. Use of the independent whole number strategy was also observed for middle-school students by Siegler and his colleagues for addition and different-denominator subtraction (Siegler et al., 2011). Adults were less likely to use this strategy than middle-school students (adults $=5 \%$ vs. middle school students $=29 \%$ ), but nevertheless, it was the most frequently selected incorrect strategy on addition and subtraction problems for both groups.

Wrong fraction operation strategy. The wrong fraction operation strategy was used on multiplication and division problems. Solvers maintained the 
denominator of both fractions and applied an arithmetic operation to the numerators of the fractions. For example, on the multiplication problem $3 / 5 \times 2 / 5$, they multiplied the numerators $3 \times 2$ to obtain the answer 6 , and maintained the value of the denominator 5 to obtain the answer $6 / 5$. On the division problem $3 / 5 \div 1 / 5$, solvers divided the numerators $3 \div 1$ to obtain the answer 3 and maintained the value of the denominator 5. Solvers then gave the answer " $3 / 5$ ". The wrong fraction operation strategy was seldom used when adding and subtracting fractions.

Solvers' use of the wrong fraction operation strategy on multiplication and division is shown in Figure 4.5. The percentage use of the wrong fraction operation strategy was analyzed in a 2 (operation: multiplication, division) $\times 2$ (solver group: conceptual, procedural $) \times 2$ (denominator: same, different $)$ mixed ANOVA. The strategy was used more frequently by conceptually- than procedurally-stronger solvers $(5.0 \%$ vs. $3.1 \%), F(1,675)=4.38, p=.04, \eta^{2}=.01$; for multiplication than division problems $(6.3 \%$ vs. $1.8 \%), F(1,675)=45.19, p<.001, \eta^{2}=.06$, and for same denominator than different denominator problems $(5.8 \%$ vs. $2.3 \%), F(1,675)=$ $63.68, p<.001, \eta^{2}=.09$. As shown in the figure, the pattern was similar across groups and operations, although it was almost never used on different-denominator division problems. 
Figure 4.5. Average frequency of selections (in \%) for the wrong fraction operation strategy. Error bars are 95\% CI calculated according to Jarmasz and Hollands (2009).
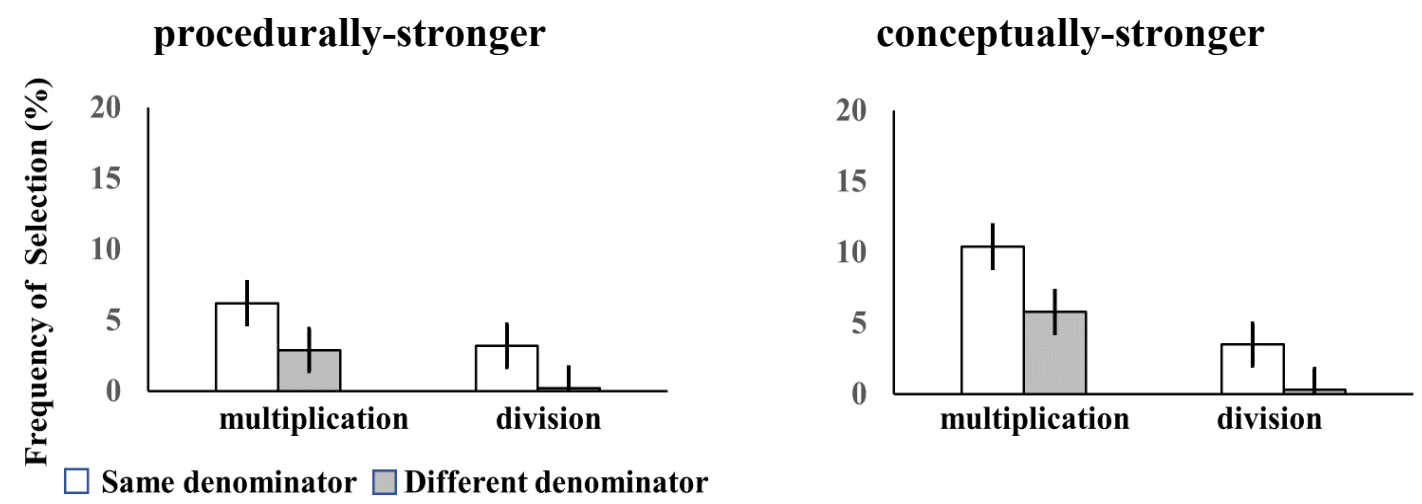

When multiplying different denominator fractions, some solvers performed a variant of the wrong fraction operation strategy. Solvers first attempted to find the common denominator then executed the wrong fraction operation strategy by multiplying the numerators and maintaining the denominators. For example, on the different- denominator problem $3 / 5 \times 1 / 2$, solvers found a common denominator for both fractions that is, $6 / 10 \times 5 / 10$. They then multiplied the numerators of the converted problem $6 \times 5$ while maintaining the value of the denominator 10 to obtain the solution 30/10. This fraction was then reduced to obtain the solution 3. Finding a common denominator is an unnecessary step on multiplication problems and may reflect procedural knowledge that is not differentiated by operation.

To summarize, conceptually-stronger solvers were more likely to select the wrong fraction operation strategy than the procedurally-stronger solvers. Furthermore, the strategy was selected more frequently when problems shared the same denominator than when they had different denominators, suggesting that fractions with the same denominator acted as a cue to maintain the value of the common denominators. However, some solvers may have converted fractions to the same 
denominator, and then incorrectly multiplied the numerators, maintaining the denominator. Finally, because strategies were inferred from solvers' responses, solvers might have selected the wrong fraction operation strategy and also made a computational error. Such responses would not have been classified as arising from the use of the wrong fraction operation strategy. More detailed information about the steps used in solving the problems would allow more complete classification of strategy use.

In summary, solvers selected the wrong fraction operation strategy when multiplying compared to dividing fractions and for same- than different-denominator problems. The strategy was also more likely to be selected by conceptually-stronger than procedurally-stronger solvers. Siegler et al. (2011) found the same pattern of results by operation and denominator relations for middle-school students. However, middle-school students selected the wrong fraction operation strategy more frequently than adults ( $4 \%$ for adults vs. $21 \%$ for middle school students).

Wrong inversion strategy. The wrong inversion strategy was the most common strategy error for same-denominator division problems. When applying this strategy, solvers inverted the first rather than the second fraction before multiplying the numerators and denominators. For example, when presented with the problem $3 / 5$ $\div 1 / 5$, solvers inverted the first fraction $3 / 5$ to obtain $5 / 3$ and then multiplied the numerators $5 \times 1=5$ and denominators $3 \times 5=15$ to obtain $5 / 15$.

Solvers' use of the wrong inversion strategy is shown in Figure 4.6. The percentage use of the wrong fraction operation strategy was analyzed in a 2 (operation: multiplication, division $) \times 2($ solver group: conceptual, procedural $) \times 2$ (denominator: same, different) mixed ANOVA. There was no significant difference in strategy selection between the conceptually- and procedurally-stronger groups 
$(5.0 \%$ vs. $3.1 \%), F(1,675)=1.33, p=.25$. The strategy was more frequently selected for division than multiplication $(7.4 \%$ vs. $2.8 \%), F(1,675)=35.31, p<.001, \eta^{2}=.05$, and more frequently for same- than different-denominator problems (7.9\% vs. $2.3 \%)$, $F(1,675)=136.53, p<.001, \eta^{2}=.17$. These main effects were qualified by the interaction between operation and denominator, $F(1,675)=113.82, p<.001, \eta^{2}=$ .14. Post-Hoc comparisons revealed that when solvers divided fractions, inverting the wrong fraction was more frequently selected for same- than different-denominator problems $(12.6 \%$ vs. $2.1 \%), p<.05$. There were no differences for multiplying sameversus different-denominator problems (2.5\% vs. $3.1 \%)$.

Figure 4.6. Average frequency of selections (in \%) for the wrong fraction inversion strategy. Error bars are 95\% CI calculated according to Jarmasz \& Hollands (2009).

procedurally-stronger

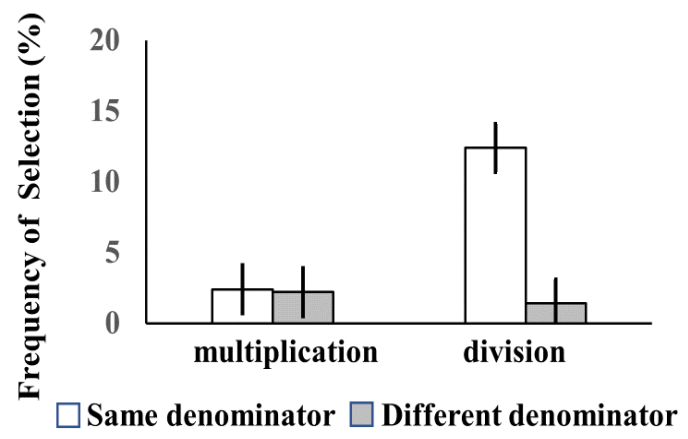

conceptually-stronger

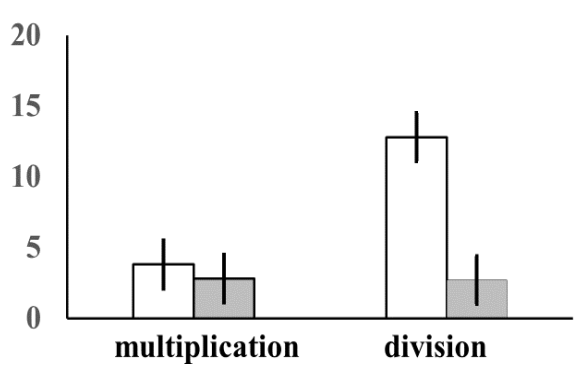

The wrong inversion strategy was frequently selected on same denominator division. Both conceptually-stronger and procedurally-stronger solvers were equally likely to incorrectly invert the wrong fraction. Solvers also occasionally inverted the first fraction of the problem when multiplying problems. These results suggest that people remember that sometimes one fraction needs to be inverted, but occasionally apply inversion to the wrong operation, and more frequently to the wrong operand in division problems. The wrong inversion strategy was not a common strategy error in middle-school students (Siegler et al. 2011; Siegler \& Pyke, 2013). Instead, most 
middle school students performed the wrong fraction operation strategy where solvers either subtracted or divided numerators while maintaining denominators (Braithwaite et al., 2017).

No response. Participants sometimes failed to respond at all. Figure 4.7 presents the average frequencies of problems that were left blank by solver group, operation and denominator relationship. The percentage use of the "blank" solutions was analyzed in a 4 (operation: addition, subtraction, multiplication, division) $\times 2$ (solver group: conceptual, procedural $) \times 2$ (denominator: same, different $)$ mixed ANOVA. There was a main effect of operation, $F(3,673)=31.71, p<.001, \eta^{2}=13$. Solvers left answers blank most frequently for division (12.3\%), followed by subtraction (4.5\%) and multiplication (4.6\%) and least frequently for addition (3.0\%). Post-hoc comparisons revealed significant differences of frequencies corresponding to these groupings (division vs. subtraction/multiplication vs. addition), $p s<.05$

Figure 4.7. Average frequency of blank answers (in \%). Error bars are 95\% CI calculated according to Jarmasz and Hollands, 2009.
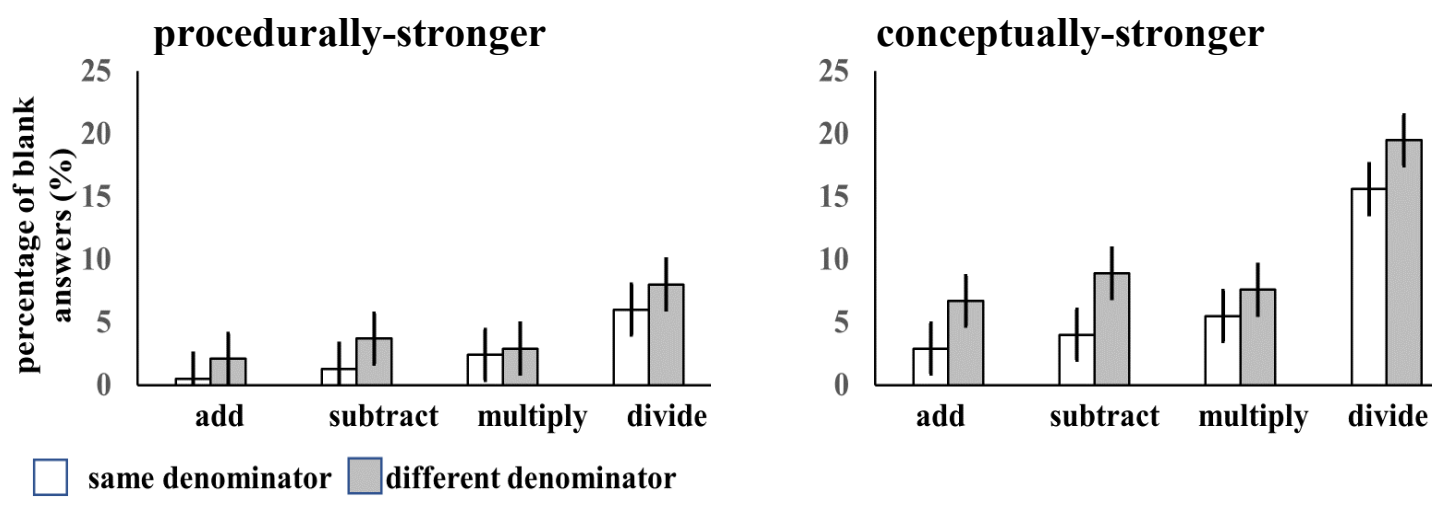

Conceptually-stronger solvers were more likely to leave answers blank than procedurally-stronger solvers $(8.8 \%$ vs. $3.4 \%), F(1,675)=24.81, p<.001, \eta^{2}=.04$. Solvers left answers blank more frequently on different- than on same- denominator problems $(7.4 \%$ vs. $4.8 \%), F(1,675)=38.96, p<.001, \eta^{2}=.06$. There was an interaction between solver group and operation, $F(3,673)=5.09, p<.005, \eta^{2}=0.02$. 
When dividing fractions, conceptually-stronger solvers left more answers blank than procedurally-stronger solvers $(17.6 \%$ vs. $7.0 \%), p<.05$. There was also an interaction between solver group and denominator, $F(1,675)=5.98, p=.02, \eta^{2}=0.01$. On different denominator problems, conceptually-stronger solvers left more answers blank than procedurally-stronger solvers $(10.7 \%$ vs. $4.2 \%), p<.05$. To summarize, blank answers reflected solvers difficulties in solving problems. Conceptuallystronger solvers experienced greater difficulty with fraction arithmetic problems than conceptually-stronger solvers.

Other than failing to respond at all, the remaining responses provided for each problem were not frequent (i.e., used by less than $5 \%$ of all participants, usually less than $1 \%$ ) and were not systematic. On these problems, solvers may have made a computational error in the process of applying an algorithm or guessed the solution.

\section{Discussion}

This chapter describes adults' performance on a set of fraction arithmetic problems used by Siegler and his colleagues for middle-school students. I analyzed the frequency of the most common errors and the types of strategies that adults selected. My goal was to determine which factors influence strategy selection. In Chapter 3, adults were profiled as conceptually-stronger and procedurally-stronger solvers. In this section, I address the question of the factors that influence strategy selection by comparing the effects of solvers' fraction knowledge, operation and denominator relationship on solvers' accuracy and their frequency of each type of strategy error. I then highlight solvers' selection of the reduction procedure, identify possible limitations to Study 1, and propose a follow-up study to address these limitations. 


\section{What are the Factors that Influence Adults to Select Correct Strategies?}

Accuracy rates obtained on all four arithmetic operations support findings of previous studies (Braithwaite et al., 2017; Siegler et al., 2011; Siegler \& Pyke, 2013). First, accuracy followed similar patterns to $6^{\text {th }}$ and $8^{\text {th }}$ grade school students in that adults had higher accuracy rates for fraction addition, subtraction and multiplication than fraction division. Second, performance for fraction arithmetic was dependent on whether fraction pairs have equal denominators. Adults were more accurate for samethan different-denominator problems but only for addition and subtraction. These patterns are comparable with $6^{\text {th }}$ and $8^{\text {th }}$ grade students (Siegler et al., 2011). In the current study I compared performance of two groups of solvers. Overall, procedural solvers performed better on fraction arithmetic problems than conceptual solvers for all fraction arithmetic operations, and there were no interactions between solver profile and denominator relationship on accuracy rates.

\section{What Incorrect Strategies do Adults Apply when they Generate Systematic}

\section{Errors?}

Adults generally used the same correct strategies as children, however, they used two other correct strategies that were used infrequently by middle-school students. First, many adults correctly used reduction to solve same denominator division. Second, some solvers converted fractions into decimals, either performing the required operation on the converted decimals or converting the solution into a decimal. Middle-school students did not give decimal answers (Braithwaite et al 2017, Siegler \& Pyke, 2013; Siegler, et al., 2011). It is possible that these differences reflect instructions provided to the adult sample, however as participants were only told to fill in the blanks with a suitable answer, and not specifically instructed about whether they needed to provide their solutions in fraction or decimal notation. 
Solvers used the independent whole number strategy most often on addition and different denominator subtraction problems and rarely on multiplication and division. When solvers used this strategy, they treated numerators and denominators as whole number components that could be added or subtracted using whole number procedures. Thus, it appeared that they failed to understand the relation between the numerator and denominator. Solvers' used the wrong operation strategy most frequently on multiplication problems with the same denominators, suggesting that the same denominator problems cued the 'common denominator' procedure; however, they still multiplied the numerators (e.g., for $3 / 5 \times 2 / 5$ they responded with $6 / 5$ not with 5/5). Use of these strategies reflects an underlying misconception about the conditions under which the strategy needs to be applied.

People used the wrong inversion strategy most frequently on division problems with the same denominators. Solvers were able to successfully identify the operation that required inversion (i.e., fraction division) but not the rule about which operand to invert. The observation that people were cued by same denominator to invert the first fraction, but applied other operations for different denominator problems, suggests that division together with a common denominator relationship acted as a cue to invert a fraction, even if solvers had difficulty recalling which operand to invert. Solvers understood that they had to invert one of the fractions when they performed fraction division, but incorrectly inverted the first instead of the second fraction. Because children had more recently learned division, they may have remembered which operand to invert whereas the adults in the present study may have forgotten this step in the procedure. 


\section{What are the Factors that Influence Adults' Selection of Incorrect Strategies?}

The FARRA computational model proposed by Braithwaite and his colleagues (2017) learned to select incorrect strategies for performing fraction arithmetic after the model was presented with problems. FARRA's strategy selection was cued by a combination of the required operation and the denominator relationship between operands. Results from Study 1 confirm that the required operation and the denominator relationship interact to influence selection of incorrect but systematic strategies among adults. Furthermore, I found that solvers who are profiled as conceptually stronger were more susceptible to the same strategy errors shown by middle-school students compared to procedurally-stronger solvers.

Solvers who selected the independent whole number strategy treated each component as separate. However, solvers selected the strategy for addition and for different denominator subtraction, but not for same denominator subtraction. Applying the independent whole number strategy to same denominator subtraction would have resulted in fractions with denominators of 0 , which was not frequently produced. Thus, solvers must have used their knowledge of fractions to realize that this strategy was not plausible and used some other strategy such as subtracting numerators while maintaining denominators (See Appendix H).

The pattern observed for the wrong fraction operation strategy was opposite to the pattern observed for the independent whole number strategy for addition and subtraction. Whereas the independent whole number strategy was more frequently selected for different denominator than same denominator problems, the wrong fraction operation strategy was selected more frequently for same denominator than different denominator problems. These patterns of errors for the two strategies suggests that some solvers were cued by both the denominator relationship and the 
operation when selecting strategies. The steps of applying the operation on the numerator but maintaining the denominator would have been correct for addition and subtraction, but incorrect for multiplication and division. Whereas, applying the required operation on both numerators and denominators would have been correct for multiplication, but not addition and subtraction.

Use of the wrong inversion strategy was associated with difficulty of retrieving and applying inversion to the appropriate fraction rather than retrieval of the inversion procedure itself. I speculate that this error was activated by a combination of noticing that same-denominator problems facilitated both the inversion and the reduction algorithm, encouraging solvers to invert fractions. For different denominator problems, the identical numerator-denominator pair was absent. As a result, the reduction algorithm was not as strongly cued as for same denominator problems. For different denominator problems, solvers instead selected other strategies.

Conceptually-stronger solvers selected the independent whole number and wrong fraction operation strategies more frequently than procedurally-stronger solvers, suggesting that solvers' knowledge of fractions was also a factor in their choice of strategy. These strategy errors were selected more frequently for conceptually- than procedurally-strong solvers, possibly because the former group had difficulty identifying the appropriate conditions required to apply the correct algorithm. Conceptually-stronger solvers were susceptible to the relationship between denominators, as well as the operation, whereas procedurally-stronger solvers had less difficulty identifying the conditions for the correct algorithms, and were less susceptible to the relationship between denominators when selecting a strategy.

There was no difference in procedurally- and conceptually-stronger solvers' 
selection of the wrong inversion strategy. However, both groups of solvers selected the strategy most frequently when dividing fractions with same denominators, indicating that selection of this strategy might not necessarily be accounted for by solvers difficulties in identifying the conditions for division, but rather accounted for by the difficulty in retrieving the procedure. Solvers who selected this strategy were able to recall that inverting a problem was part of the strategy, but incorrectly inverted the first rather than the second fraction. Note that after inversion, the inverted problem would have an identical numerator-denominator pair, which would be a condition for the cross-reduction algorithm. I speculate that this error was activated by a combination of noticing that same denominator problems facilitated both the inversion and the reduction algorithm, encouraging solvers to invert fractions. For different denominator problems, the identical numerator-denominator pair after inversion was absent, as a result the reduction algorithm was not as strongly cued as for same denominator problems. For different denominator problems, solvers instead selected other strategies such as performing another fraction operation (See Appendix G).

In summary, strategy selection was cued by a combination of three factors operation, denominator relationship and solvers' fraction knowledge. Solvers who made strategy errors appeared to be cued by the required operation but were also sensitive to the relationship between denominators. Conceptually-stronger solvers were more susceptible to denominator relationship than procedurally-stronger solvers, possibly because procedurally-solvers had more direct access to the correct procedures for most operations.

\section{Fraction Arithmetic and Fraction Reduction}

A key issue of this dissertation is the identification of the factors that facilitate 
solvers to select and apply reduction procedures. Even with the limited number of reducible problems presented to participants in the FARRA portion of the assessment, some solvers understood the concept of fraction reduction and how to apply reduction correctly. However, there were not enough problems that allowed reduction in the FARRA data set to make more detailed conclusions about how or when participants used reduction.

\section{Limitations}

Solvers' responses were collected through an online study. The online mode of collecting responses is efficient for collecting responses from a large number of participants. However, the online method was also unreliable in several ways, which limited my ability to compare errors and infer strategies for solving fraction arithmetic problems. First, there was a lack of control for the conditions under which problems were solved. Participants could have solved the problems through various ways (i.e., using a calculator, using worked protocols or performing mental arithmetic without any external aid).

Secondly, responses were collected and analyzed, but strategies were inferred from their responses. There was no way of confirming the strategies that solvers selected to solve problems or identifying whether errors were a result of selecting an incorrect strategy or a computational error when applying a strategy.

Another limitation of the study is the difficulty in drawing specific conclusions about how the relation between denominators and the required operation might influence participants' use of reduction. In the FARRA portion of the assessment, the only reducible fraction multiplication problem had different denominators. Conversely, all the reducible fraction division problems had common denominators. Findings from this study indicate that the relation between 
denominators cued specific strategies. However, reducible problems for samedenominator multiplication and different-denominator division were not presented to solvers. The question of whether denominator relation cues fraction reduction after controlling for the required operation also needs to be established.

\section{The Solution: A Follow-up Study}

Study 2 was designed to address the limitations of Study 1. I invited participants to solve fraction problems in a laboratory setting and obtained detailed written protocols showing how they solved each problem. Furthermore, in Study 2, I also tested whether the factors identified in Study 1 - fraction knowledge, fraction operation, and denominator relations -- determine whether solvers are likely to select and apply reduction. 


\section{CHAPTER 5: STUDY 2 (PART 1: WRITTEN PROTOCOLS)}

Study 2 focused on solvers' selection and application of reduction during fraction arithmetic. In this chapter, I address the questions "What are the problem characteristics and solver profiles that facilitate solvers to reduce fractions?" In Chapter 6, I address the questions "Are reducible fraction multiplication problems solved more accurately and quickly than non-reducible problems? and "Where do solvers focus their attention when they multiply fractions?"

\section{Overview of Study 2}

Figure 5.1 illustrates how participants' characteristics and solver profiles across each study and across parts and cohorts for Study 2. Study 2 was divided into two parts and participants were assigned to two separate cohorts. All participants solved fraction arithmetic problems and were requested to provide written protocols in the first part of Study 2. In the second part of the study, participants were separated into two cohorts and multiplied fractions. Response times and latencies were recorded for both cohorts. However, ocular measures were only recorded for one cohort (eyetracking cohort). Ocular measures were not recorded for the other cohort (non eyetracking cohort). Recording ocular measures requires participants to be positioned in potentially uncomfortable positions (i.e., rest their chin on a chinrest). In contrast, in research studies in mathematical cognition, participants are typically not required to be restricted in their body position. The different cohorts ensure that collected accuracies and response times are not due to a confound of participants' discomfort.

Figure 5.1 also illustrates how the sample size of each study varied. All participants who completed Study 1 were invited to participate in Study 2. However, only 59 participants consented to participate in Study 2. All these participants completed the written protocol part of the study. However, data from three 
participants were excluded due to apparatus failure to collect data. Data from a further 5 participants were excluded from response time and ocular measure analysis as they were not able to multiply fractions for more than $50 \%$ of the presented problems.

These exclusion criteria will be elaborated upon further in the corresponding sections of Chapters 5 and 6.

Figure 5.1: Participant characteristics across Studies 1 and 2; and across parts and cohorts for Study 2.

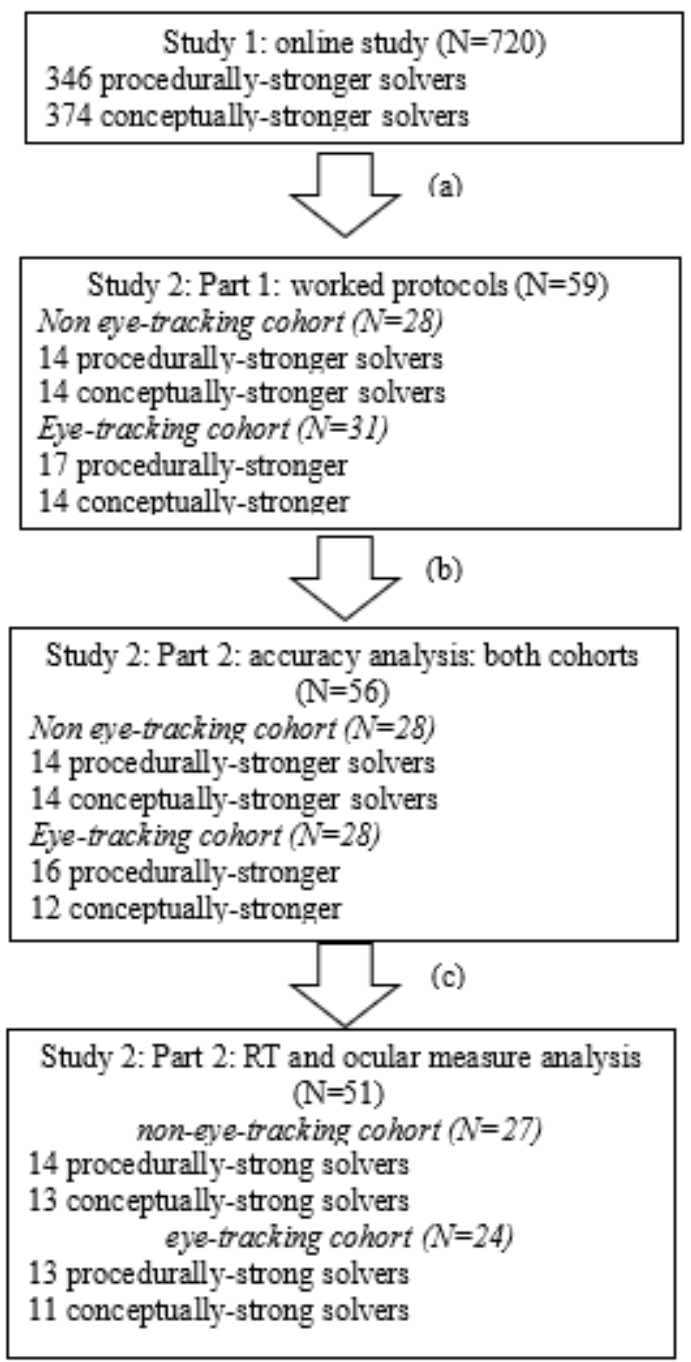

(a): All participants who completed Study 1 were invited to participate in Study 2 for course credit or a stipend of $\$ 15$, but 59 participants agreed to participate

(b): Voice recordings were not adequately captured for 3 participants. Data for these participants were excluded from the analysis

(c): 5 participants who provided less than $50 \%$ correct answers and were excluded from the analysis 


\section{Hypotheses}

For Part One of Study 2, I tested the hypotheses that the factors that cue strategies -- operation, denominator relationship, and solver knowledge -- also cue reduction. There has not been any previous research, thus I based my hypotheses on previously identified problem characteristics that cued reduction - namely required operation and whether denominators within the problem shared the same denominator (Siegler et al, 2011; Braithwaite et al., 2017). If the identified factors do indeed cue the reduction strategy, I expected that:

(1) Frequency of reduction use will differ across problems..

(2) Frequency of reduction use will differ for same- than for differentdenominator problems.

I also identified two different fractions profiles in Chapter 3. In Chapter 4, conceptually-stronger solvers made strategy errors more frequently than procedurallystronger solvers. Thus, solvers' relative knowledge of fractions concepts and procedures might also be related to selection of the reduction procedure. If this were the case, I should also expect that:

(3) Frequency of reduction use will differ across conceptually- and procedurally-stronger solvers.

\section{Method}

\section{Participants}

All respondents in Study 1 were contacted by email and invited to participate in Study 2. Participants were informed they could choose between either course credit or a stipend of $\$ 15.00$ for completing the study. Fifty-nine participants volunteered to participate in Study $2(M d n$ age $=19$ years; $52.5 \%$ female $)$. These participants were randomly assigned to either the eye-tracking or the non-eye-tracking cohort. Of these 
participants, 28 were conceptually-stronger solvers and 31 were procedurally-stronger solvers as described in Chapter 3. In the non-eye-tracking cohort, there were 14 conceptually-stronger and 14 procedurally-stronger solvers. In the eye-tracking cohort, there were 14 conceptually-stronger and 17 procedurally-stronger solvers.

\section{Materials}

Thirty fraction arithmetic problems (shown in Table 5.1) were presented in Part One of Study 2. To study whether people would use reduction, I used a larger number of multiplication problems than those from other operations (see Table 5.1). Three problems had one reducible fraction and different denominators (row 1 in Table 5.1). Three other problems had one reducible fraction and the same denominators (row 3 in Table 5.1). Three multiplication problems were created (row 2) by swapping denominators of the row 1 problems; these were cross-reducible problems. I also modified the three problems with same denominators, such that both fractions had the same denominators with a multiple of 5 (row four) and thus had one reducible operand.

Addition, subtraction and division problems with the same fractions as the reducible multiplication problems were also presented to participants. These problems were used to verify whether participants would select and apply the same strategies identified in Chapter 4. Note that reduction could be used for some of these addition and subtraction problems if participants recognized that reduction would lead to a common denominator. For example, $3 / 4+5 / 20$ could be efficiently solved if $5 / 20$ was reduced to $1 / 4$. 
Table 5.1. Fraction arithmetic problems in Part 1 of Study 2

\begin{tabular}{|c|c|c|c|c|}
\hline Denominators & Addition & Subtraction & Multiplication & Division \\
\hline \multirow[t]{6}{*}{ Different } & $3 / 4+5 / 10$ & $3 / 4-5 / 10$ & $3 / 4 \times \mathbf{5} / \mathbf{1 0}$ & $3 / 4 \div 5 / 10$ \\
\hline & $2 / 3+5 / 15$ & $2 / 3-5 / 15$ & $2 / 3 \times \mathbf{5} / \mathbf{1 5}$ & $2 / 3 \div 5 / 15$ \\
\hline & $3 / 4+5 / 20$ & $3 / 4-5 / 20$ & $3 / 4 \times \mathbf{5} / \mathbf{2 0}$ & $3 / 4 \div \mathbf{5} / \mathbf{2 0}$ \\
\hline & & & $5 / 4 \times 3 / 10$ & \\
\hline & & & $5 / 3 \times 2 / 15$ & \\
\hline & & & $5 / 4 \times 3 / 20$ & \\
\hline \multirow[t]{6}{*}{ Same } & $3 / 10+2 / 10$ & $3 / 10-2 / 10$ & $3 / 10 \times \mathbf{2} / \mathbf{1 0}$ & $3 / 10 \div 2 / 10$ \\
\hline & $3 / 15+2 / 15$ & $3 / 15-2 / 15$ & $\mathbf{3} / \mathbf{1 5} \times 2 / 15$ & $\mathbf{3} / \mathbf{1 5} \div 2 / 15$ \\
\hline & $4 / 20+3 / 20$ & $4 / 20-3 / 20$ & $4 / 20 \times 3 / 20$ & $4 / 20 \div 3 / 20$ \\
\hline & & & $3 / 10 \times \mathbf{5} / \mathbf{1 0}$ & \\
\hline & & & $2 / 15 \times 5 / 15$ & \\
\hline & & & $3 / 20 \times \mathbf{5} / \mathbf{2 0}$ & \\
\hline
\end{tabular}

Note. Items in the first and third rows were matched by operands across operations.

Multiplication items in the second and fourth rows were created by swapping numerators of items in the first and third rows. Reducible operands are in bold for multiplication and division.

Different-denominator multiplication problems included only one reducible fraction and were not ideal to study whether participants would cross-reduce numerator-denominator pairs from different fractions. Thus, I included three crossreducible multiplication problems with different denominators by switching the numerators of the different denominator fraction multiplication problems (e.g., $3 / 10 \times$ $2 / 10=6 / 100)$. These problems had the same solution as the original problems (e.g., $2 / 10 \times 3 / 10=6 / 100)$. The fraction with the larger value was maintained as the first fraction in the problem. This restriction ensured that the correct solution for fraction subtraction was greater than 0 , and that the location of the larger fraction was controlled for all problems. As shown in Table 5.2, addition, subtraction, and division 
problems with the same fractions as the reducible multiplication problems were also presented to participants. These problems were used to verify whether participants would select and apply the same strategies identified in Chapter 4.

\section{Procedure}

At the start of each study, participants were told that the study was in two parts. Participants were informed that in the first part, they would solve fraction arithmetic problems. Participants were informed that they would be presented with a fraction arithmetic problem and were told to "solve each problem and use the provided paper to show how you solved each problem." Participants were also told to "provide as much detail as they were able" about how they solved each problem. They were then required to verbally describe how each problem was solved. Verbal descriptions of each problem were recorded. The researcher did not interact with the participant while they were providing the worked protocol. If participants asked whether they needed to reduce or simplify a fraction, they were told "it is up to you." The 30 problems from Table 5.2 were presented in a random order for each participant.

\section{Results}

\section{Descriptives and Group Comparisons}

Group membership (i.e., procedurally vs. conceptually strong) was determined from Study 1. Table 5.2 presents means and standard deviation scores for conceptually-stronger and procedurally-stronger solvers for all measures. Group performance was compared with independent samples t-tests. The conceptuallystronger solvers performed better on the conceptual measure than the procedurallystronger solvers, as expected given the results of Study 1. However, there were no group differences on the procedural fraction measure. Thus, these subgroups are not 
fully representative of the overall classifications from Study 1 despite being members of those groups.

Conceptually-stronger solvers performed slightly better than procedurallystronger solvers on multi-digit addition, but there were no differences between solvers on multi-digit subtraction or multiplication. Furthermore, there were no differences between groups on single-digit multiplication. Overall, the trend was that the conceptually-stronger group had slightly better arithmetic scores, better conceptual knowledge, and comparable procedural knowledge than the procedurally-stronger group. Thus, any differences across conceptually- and procedurally-stronger groups can be attributed to differences in fraction knowledge rather than in their arithmetic ability. 
Table 5.2: Means and standard deviations of conceptually-stronger and procedurally-stronger solvers. Conceptual and procedural scores are the standardized residuals.

\begin{tabular}{|c|c|c|c|c|c|c|}
\hline \multirow[t]{2}{*}{ Measure } & \multicolumn{2}{|c|}{$\begin{array}{l}\text { Procedurally } \\
\text { - stronger } \\
(n=32)\end{array}$} & \multicolumn{2}{|c|}{$\begin{array}{l}\text { Conceptually- } \\
\text { stronger } \\
(n=27)\end{array}$} & \multicolumn{2}{|c|}{ Comparison } \\
\hline & $M$ & $S D$ & $M$ & $S D$ & $d f$ & $t$ \\
\hline BMT (large) & 16.5 & 7.2 & 17.4 & 11.4 & 57 & 0.36 \\
\hline BMT (small) & 30.5 & 12.2 & 34.1 & 11.2 & 57 & 1.21 \\
\hline CFT (add) & 9.6 & 3.5 & 11.8 & 4.1 & 57 & $2.27^{*}$ \\
\hline CFT (subtract) & 8.5 & 2.9 & 10.1 & 4.1 & 57 & 1.77 \\
\hline CFT (multiply) & 5.5 & 2.9 & 6.4 & 4.0 & $56^{\mathrm{a}}$ & 0.98 \\
\hline FKA (Conceptual) & 71.2 & 13.7 & 86.6 & 11.9 & 57 & $4.58 * *$ \\
\hline FKA (Procedural) & 80.4 & 21.1 & 84.7 & 25.5 & 57 & 1.57 \\
\hline FARRA & 84.7 & 20.5 & 81.3 & 22.3 & 56 & -0.62 \\
\hline $\begin{array}{l}\text { Residualized } \\
\text { conceptual score }\end{array}$ & 0.08 & 0.1 & 0.90 & 0.1 & 57 & $-4.12 * *$ \\
\hline $\begin{array}{l}\text { Residualized } \\
\text { procedural score }\end{array}$ & 0.82 & 0.1 & 0.40 & 0.2 & 57 & 2.11 \\
\hline
\end{tabular}

Note: One procedurally-stronger solver did not provide any answers for the FARRA problems in Study 1.

\section{Accuracy on Experimental Trials}

Participants provided written protocols for each problem shown in Table 5.1. A problem was scored as correct if the correct solution was provided in either fractions or decimals (correct to one decimal place). Mean percentage accuracy for each participant was calculated in each condition defined by operation and denominator relation (same, different). Thus, there were three items per condition for each of fraction addition, subtraction and division and six items for multiplication. 
Overall, participants provided accurate answers, with a mean percentage correct of $87.2 \%$. Participants' performance on written protocol problems was similar to their performance on the online study $(M=83.0 \%)$

Figure 5.2 shows average percentage scores obtained by each group of solvers across conditions. Accuracy scores (percentage correct) were analyzed in a mixed ANOVA with 2 (group: procedurally-stronger vs. conceptually-stronger) as a between-groups factor, 2 (denominator: same vs. different), and 4 (operation: addition, subtraction, multiplication, division) as repeated measures. Post-hoc comparisons were tested using Tukey HSD at .05 level of significance.

Figure 5.2: Percentage scores obtained by procedurally-and conceptually-stronger solvers by operation (addition, subtraction, multiplication and division) and denominator relationship (same vs. different). Error bars are 95\% CI calculated according to Jumasz \& Holland (2009).
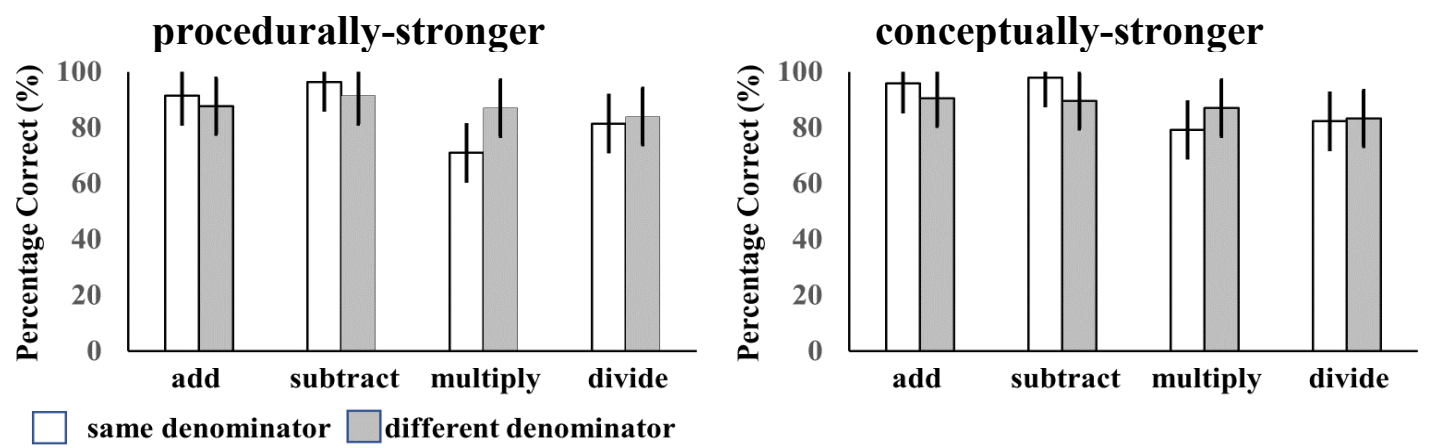

There was a significant main effect of operation, $F(3,55)=5.34, p<.005, \eta^{2}=$ .22. Participants performed better on subtraction (93.8\%) and addition (91.4\%) than on multiplication $(81.0 \%)$, and division $(82.8 \%)$, but the only significant pairwise difference was between subtraction and multiplication $(p<.05)$. There was also a significant interaction between operation and denominator, $F(3,55)=6.15, p<.005$, $\eta^{2}=.25$. Specifically, solvers found same-denominator problems more difficult to 
solve than different-denominator problems for multiplication $(75.4 \%$ vs. $87.0 \%, p<$ $.05)$, but not for the other operations. There was no overall difference in performance between conceptually- and procedurally-stronger solvers $(88.2 \%$ vs. $86.3 \%), F(1,57)$ $=0.16, p=.69$, and patterns of performance were similar in the two groups, as shown in Figure 5.2. In summary, despite overall high levels of performance, participants nevertheless made errors on about $10 \%$ of subtraction and addition problems and about $20 \%$ of multiplication and division problems. The written protocols were analyzed to provide further details about the sources of these errors. First, however, I describe the frequency with which participants used reduction in their solutions to these problems.

\section{Analysis of Written Protocols}

Participants' protocols included three reduction related strategies. First, if solvers did not apply the reduction algorithm these problems were coded as "notreduced" for that solver. Second, if a solver applied the reduction algorithm before obtaining the solution, the solution was coded as the reduction-before-solution strategy. Finally, if a solver applied reduction after obtaining a solution the solution was coded as reduction-after-solution strategy. For example, for the multiplication problem $3 / 4 \times 5 / 10$, protocol (1) was coded as "not reduced", protocol (2) was coded as "reduction-before-solution" and protocol (3) was coded as "reduction-aftersolution”.

(1) $3 / 4 \times 5 / 10=15 / 40$

(2) $3 / 4 \times 5 / 10=3 / 4 \times 1 / 2=3 / 8$

(3) $3 / 4 \times 5 / 10=15 / 40=3 / 8$

Responses to each problem and the percentage of participants within each group that provided each response are presented in Appendix H. The corresponding 
tables indicate whether the solution was reduced. When responses could not be categorized into any of the identified strategies, participants' verbal and written protocols were further analyzed to determine how they obtained each solution. A descriptive analysis of protocols is provided in the subsections "Reduction Use" and "Incorrect Responses" later in the chapter.

\section{Reduction Use}

Participants' average frequencies of reduction use to obtain correct solutions by operation and denominator relationship are presented in Figure 5.3. Mean frequencies of correct reduction use (percentage correct) were analyzed in a mixed ANOVA with 2 (group: procedurally-stronger vs. conceptually-stronger) as a between-groups factor, 2 (denominator: same vs. different), and 4 (operation: addition, subtraction, multiplication, division) as repeated measures. Post-hoc comparisons were tested using Tukey HSD at .05 level of significance Figure 5.3: Average frequency of correct reduction use obtained by procedurallyand conceptually-stronger solvers by operation (addition, subtraction, multiplication and division) and denominator relationship (same vs. different). Error bars are 95\% CI calculated according to Jarmasz and Hollands (2009).
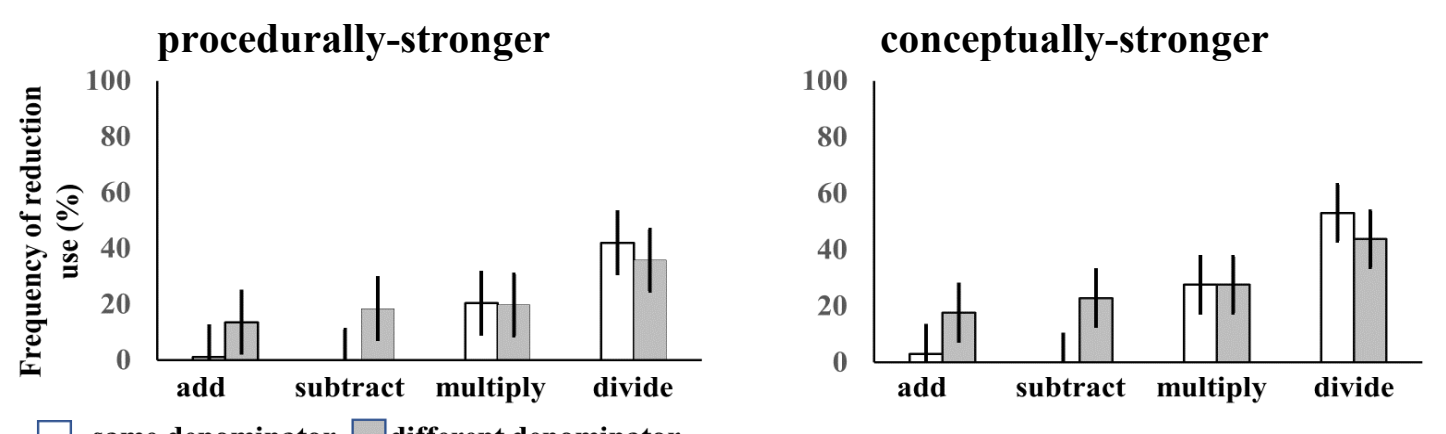

There was a significant main effect of operation, $F(3,55)=17.72, p<.005, \mathrm{\eta}^{2}$ $=.49$. Solvers used reduction more frequently on division $(43.7 \%)$ than on multiplication (23.8\%). Reduction was used least frequently on addition (8.9\%) and 
subtract (10.4\%), There were pairwise differences between division and the other three operations, and significant differences between multiplication and the other operations $(p s<.05)$. There was no difference in reduction use between addition and subtraction.

There was also a significant main effect of denominator, $F(1,57)=6.67, p$ $=.01, \eta^{2}=.11$. Solvers used reduction more frequently for different denominator $(25.0 \%)$ than same denominator problems $(18.4 \%)$. This main effect was qualified by a significant interaction between operation and denominator, $F(3,55)=10.50, p<$ $.001, \eta^{2}=.36$. Specifically, solvers used reduction for different denominator problems more frequently than same denominator problems for addition and subtraction, but not for multiplication and division. There was no overall difference in reduction use between conceptually- and procedurally-stronger solvers $(24.5 \%$ vs. $18.9 \%), F(1,57)$ $=0.84, p=0.36$, and patterns of performance were similar in the two groups, as shown in Figure 5.3.

In summary, solvers used reduction more frequently for division than multiplication, and addition and subtraction problems were reduced least frequently. Solvers also reduced different denominator problems more frequently for different denominator than same denominator problems, but only for addition and subtraction problems. The latter observation could be accounted for by the fact that reducing problems would not have led solvers to provide a correct solution for all addition and subtraction problems in the problem set, thus it was not possible to provide correctly reduced solutions for all problems.

\section{Reduction-related Strategies}

Each category of reduction-related strategies for correct solutions (nonreduced, reduction-before-solution, reduction-after-solution) was expressed as a 
percentage by operation (multiplication and division) and denominator relationship (same, different) across problem type for each participant. Addition and subtraction were not analyzed because an analysis of protocols revealed that reduction was not applied or was only applied after the solution had been obtained, when applicable. For each type of reduction strategy, Figures 5.4(a) to (c) present the average percentage of reduction use as a proportion of the total number of problems by operation and denominator relationship (i.e., 3 problems for addition, subtraction and division; 6 problems for multiplication). Three $2 \times 2 \times 2$ ANOVAs with group (procedurally strong vs. conceptually strong) as a between-subjects factor and operation (multiplication vs. division) and denominator (same vs. different) as withinsubjects factors were performed on average frequencies of each category of reduction use.

Nonreduction. Mean use of nonreduced solutions is shown in Figure 5.4a. Solvers left solutions in non-reduced form more often for multiplication than division $(37.0 \%$ vs. $17.1 \%), F(1,57)=21.79, p<.001, \eta^{2}=.28$. There were no other significant effects.

Reduction-before-solution. Figure $5.4 \mathrm{~b}$ presents the percentage of problems where solvers applied the reduction-before-solution strategy. Solvers used the strategy less often for multiplication than division $(23.8 \%$ vs. $43.7 \%), F(1,57)=26.81, p<$ $.001, \eta^{2}=.32$. No other effects were significant.

Reduction-after-solution. Figure $5.4 \mathrm{c}$ presents the percentage use of problems where solvers applied the reduction-after-solution strategy. Solvers reduced solutions more frequently for different-denominator than same-denominator problems $(27.2 \%$ vs. $15.6 \%), F(1,57)=18.30, p<.001, \eta^{2}=.24$. There was an interaction between operation and group, $F(1,57)=5.31, p=.03$. Procedurally-stronger solvers 
applied the reduction-after-solution strategy more frequently than conceptuallystronger solvers on division (29.0\% vs. $16.1 \%)$, whereas both groups of solvers applied the strategy with equal frequency for multiplication (17.2\% vs. $23.2 \%)$.

Figure 5.4. Percentage use of non-reduced and reduced solutions in written protocols. Error bars are 95\% CI calculated according to Jumasz \& Holland (2009).

(a) Non-reduced solutions

procedurally-stronger

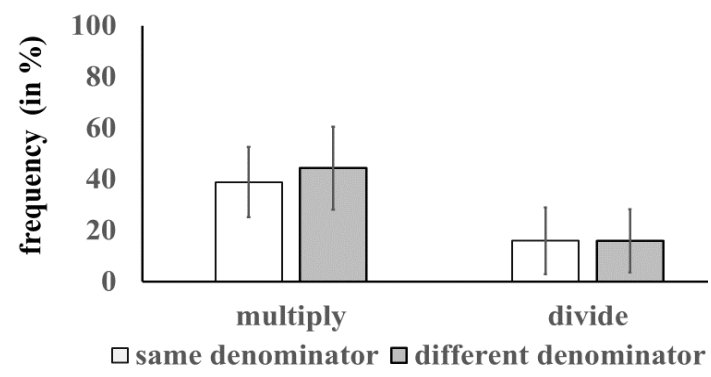

(b) Reduction-before-solution

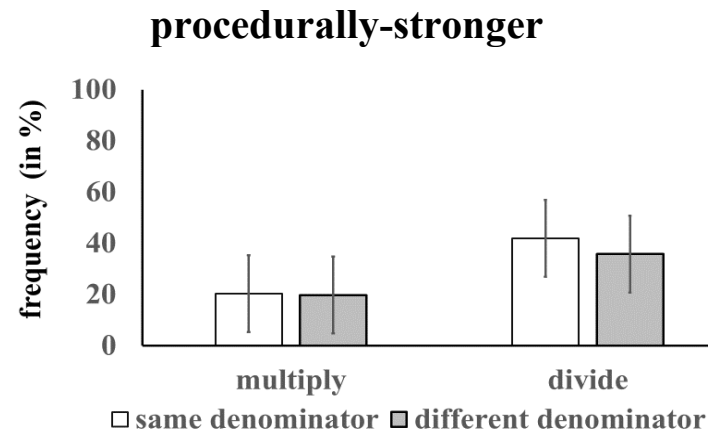

conceptually-stronger

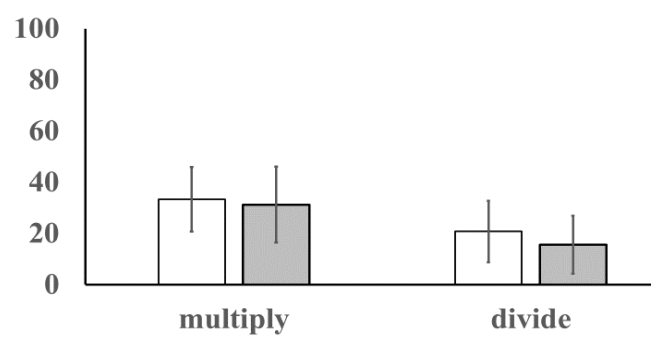

conceptually-stronger

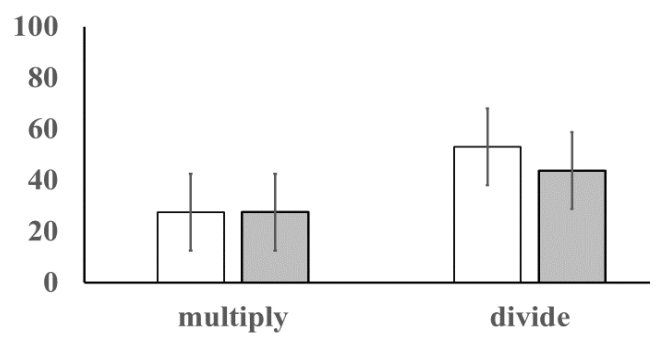


(c) Reduction-after-solution

procedurally-stronger

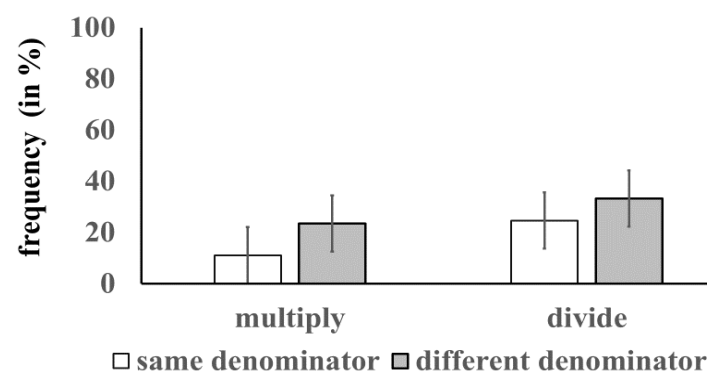

conceptually-stronger

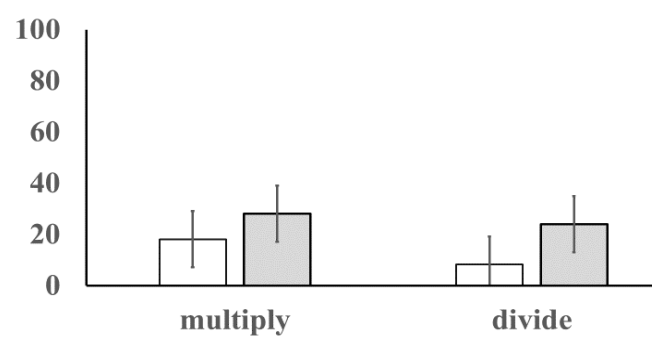

Summary. In summary, I found differences in reduction use across operation and denominator relationship, but not across fraction knowledge profiles. Even though all problems contained at least one reducible pair, solvers provided reduced solutions more frequently for division than multiplication problems, whereas they provided non-reduced solutions more frequently for multiplication than division. Solvers were also more likely to apply the reduce-before-solution procedure for division than multiplication problems. Solvers applied the reduce-after-solution equally for multiplication and division problems. These findings suggest that reduction-before-solution is cued by the division operation, possibly because inverting an operand draws attention to the common factor. On the other hand, the reduction algorithm was cued less frequently for the multiplication, possibly because the multiplication operation cued solvers to multiply numerators and denominators.

\section{Incorrect Responses}

In this section, I describe errors that solvers made while they performed fraction arithmetic. (See Appendix H for solvers' responses). Responses were first categorized according to the identified strategies described in Chapter 4. For some problems (e.g., $2 / 10 \times 3 / 10$ ), a response was provided (i.e., 5/100) that might reflect selection of the wrong strategy (i.e., the wrong fraction operation) but might also have occurred for another reason (i.e., adding the numerators and multiplying the 
denominators). If that person consistently made the same strategy selection error for other problems involving the arithmetic operations, then this ambiguous error was classified as such. However, if solvers did not consistently make this error, or demonstrated that they were able to perform the arithmetic operation for the other problems, solvers' protocols were further analyzed to categorize the error. Table 5.3 presents the percentage of total trials on which participants made specific types of errors across the four operations

Table 5.3: Percentage (of total trials) on which participants made specific types of errors grouped by operation and denominator relationship $(S D=$ Same denominator problems; $D D=$ Different denominator problems)

\begin{tabular}{lcccccccc}
\hline Type of Error & \multicolumn{2}{c}{ Addition } & \multicolumn{2}{c}{ Subtraction } & \multicolumn{2}{c}{ Multiplication } & \multicolumn{2}{c}{ Division } \\
\hline & SD & DD & SD & DD & SD & DD & SD & DD \\
\hline Independent whole & 5.2 & 6.2 & 0.5 & 4.0 & -- & 0.3 & -- & -- \\
number & & & & & & & & \\
Wrong fraction operation & -- & -- & -- & -- & 10.3 & 5.1 & 2.9 & 4.9 \\
Wrong inversion & -- & -- & -- & -- & -- & -- & 3.2 & 2.1 \\
Other systematic strategy & -- & -- & 1.9 & -- & -- & -- & -- & -- \\
Computation & 0.6 & 1.1 & -- & 0.6 & 11.3 & 4.3 & 2.5 & 3.0 \\
Number substitution & -- & 1.7 & -- & 0.5 & -- & -- & 2.3 & 1.9 \\
Operation substitution & -- & 0.6 & -- & 0.5 & 2.9 & 2.4 & 2.7 & -- \\
Combination/Unique & 0.6 & 1.7 & 0.5 & 3.8 & 1.1 & 0.9 & 4.6 & 4.0 \\
\hline Total Percentage Error & 6.4 & 11.3 & 2.9 & 9.5 & 25.7 & 12.6 & 15.0 & 13.8
\end{tabular}

Note. Unique errors were complex or combinations of errors that were used on a single problem by a given participant.

Addition and subtraction. Solvers made an independent whole number strategy error on $5.7 \%$ of addition and $2.3 \%$ of subtraction problems. These results were comparable to the frequencies that the strategy was selected in Study 1 (6.3\% for addition; $3.4 \%$ for subtraction). Four other types of errors were identified for addition 
and subtraction problems: computation $(0.6 \%)$, number substitution $(0.6 \%)$, operation substitution $(0.3 \%)$, and combinations of these errors $(1.7 \%)$.

Computation errors. Computation errors occurred when solvers applied the correct strategy but made an incorrect calculation when applying a single step. As a consequence, the remaining steps in the strategy, though correctly applied, resulted in an incorrect response. For example, presented with the problem $2 / 3+5 / 15$ :

$$
2 / 3+5 / 15=10 / 15+5 / 15=20 / 15=4 / 3
$$

The solver correctly converted the fractions in the problem into equivalent problems with the same denominator but made an error when adding the numerators $10+15$ to obtain an incorrect solution $20 / 15$. Although the solver correctly reduced $20 / 15$ to $4 / 3$, the response was incorrect.

Number substitution errors. Some solvers made errors that were cued by features of their worked protocol. I called these "number substitution" errors because solvers were cued by components provided within their protocol and substituted this component when performing a computation. For example, when presented with the problem, $3 / 4+5 / 10$, the worked protocol provided by the solver was

$$
3 / 4+5 / 10=15 / 20+20 / 20=35 / 20
$$

The solver correctly identified the common denominator for the fraction in the problem 20 and successfully converted the first fraction into an equivalent fraction $15 / 20$, but failed to convert the second fraction into its equivalent $10 / 20$, instead producing 20/20. I speculate that the solver was cued by the number 20 that the solver had previously written and incorrectly used this component in the numerator of the second operand. Similarly, when presented with the subtraction problem $2 / 3-5 / 15$, another solver was substituted the number " 5 " instead of 15 for the denominator of the answer: 


$$
2 / 3-5 / 15=10 / 15-5 / 15=5 / 5
$$

The number substitution errors did not involve selection of an incorrect strategy, but instead the wrong number was activated and substituted for the correct one at a specific step in the algorithm. These errors did not generalize to other similar problems for those people, thus there was no evidence that solvers could not perform the relevant computation.

Operation substitution errors. The third type of error involved substituting the wrong operation, and then applying the correct strategy for that operation. For example, when presented with the problem $3 / 4+5 / 10$, one participant wrote a division sign instead of an addition sign:

$$
3 / 4+5 / 10=3 / 4 \div 5 / 10=3 / 4 \times 2=3 / 2
$$

Because this solver was able to perform the other addition problems correctly, this error appears to be a random substitution, rather than an incorrect addition algorithm. Likewise, when presented with the subtraction problem 3/4 -5/10, another solver added the fractions as follows:

$$
3 / 4-5 / 10=3 / 4+1 / 2=3 / 4+2 / 4=5 / 4
$$

Combination errors. The fourth type of error involved the combination of selecting an incorrect strategy and making a computational error or a substitution error. For example, for the problem $3 / 4+5 / 10$, the solver provided the following protocol:

$$
3 / 4+5 / 10=8 / 14=2 / 7
$$

This protocol suggests the solver selected the independent whole number strategy, as the solver added the numerators $3+5=8$ and denominators $4+10$ of both fractions to obtain the solution $8 / 14$. However, the solver then incorrectly applied the reduction algorithm. Applying the reduction algorithm successfully would have 
resulted in the fraction 4/7. However, the solver provided the response 2/7.

Other errors. Other participants used idiosyncratic strategies. For example, one participant made the systematic strategy error of subtracting numerators and dividing denominators when subtracting problems with same denominators. For example, when presented with the problem " $3 / 10-2 / 10$ ", the written protocol provided by the solver was:

$$
3 / 10-2 / 10=1 / 1=1
$$

This solver provided written protocols that described identical steps for the three same denominator problems. No other solvers made this error.

Summary for addition and subtraction. To summarize, most solvers provided correct solutions on worked protocols on addition and subtraction problems. However, some solvers made strategy selection errors (i.e., the independent whole number strategy); others applied the algorithm for another fraction arithmetic operation; made a computational, number substitution, or operation substitution error; or made combination of errors. $20.3 \%$ of the 59 participants made an error on at least one addition problem, while $15.2 \%$ of the participants made an error on at least one subtraction problem.

Multiplication. Recall that participants made errors on almost $20 \%$ of multiplication problems. Along with the wrong fraction operation error $(7.8 \%)$, the other identified errors were computation errors $(7.8 \%)$, operation-substitution errors $(2.7 \%)$ and combination errors $(1.0 \%)$.

Strategy selection errors. Of solvers' errors from applying the wrong fraction operation strategy: $66 \%$ were on same denominator problems, whereas $34 \%$ were on different denominator problems. Recall that that application of the wrong fraction operation strategy required solvers to maintain denominators. For different 
denominator problems, solvers converted the problem such that both fractions had a common denominator, then multiplied the numerators while maintaining the denominator. For example:

$$
3 / 4 \times 5 / 20=15 / 20 \times 5 / 20=75 / 20
$$

More solvers selected the wrong fraction operation strategy for same than different denominator problems, possibly because they were cued by the same denominator to maintain the value of the denominator.

Procedurally-stronger solvers had difficulty multiplying the denominators of these fractions (i.e., $15 \times 15$ ) compared to denominators of $10 \times 10$ or $20 \times 20$; (errors were $3.7 \%$ and $7.4 \%$ of total responses respectively), and were likely to either make a computational error when multiplying 15 and 15 , or leave the denominator blank. Error rates for conceptually-stronger solvers that required multiplying denominators of 10 and 20 were comparable to multiplying problems with denominators of 15 (6.3\% and $6.8 \%$, respectively). This pattern was unexpected in that the two groups did not differ in either single- or multi-digit multiplication skills (see Table 5.1).

Operation-substitution errors. A few solvers also made operation-substitution errors. For example, when one participant was presented with the problem $3 / 10 \times$ $2 / 10$, they divided the fractions to obtain $3 / 2$; another participant, when presented with the problem $3 / 20 \times 5 / 10$, added the fractions to obtain $8 / 20$

Summary for multiplication errors. In summary, $61 \%$ of the 59 participants made an error on at least one multiplication problem. Of these errors, computational errors were made most frequently. Solvers made strategy selection errors, computational errors, and operation-substitution errors. Solvers were able to multiply numerators successfully, but some had difficulty multiplying denominators that were larger numbers. Only same-denominator problems had difficult calculations and thus 
the relation between denominators was confounded with computational difficulty.

Division. The categories of errors identified in the other operations were also observed when dividing fractions. Solvers made systematic strategy errors such as the wrong fraction operation (3.9\% of all responses) and the wrong fraction inversion error $(2.7 \%$ of all responses). They also made computational errors $(2.8 \%)$, number substitution errors $(2.1 \%)$ and operation substitution errors $(1.4 \%)$ or made combinations of errors $(4.3 \%)$.

Strategy selection errors. Apart from the wrong fraction operation and wrong inversion errors, many of the unique and combined errors (as described below) also included some kind of problem in selecting or applying a step in the division algorithm.

Number substitution errors. Some solvers also made number substitution errors. For example, when presented with the problem, $4 / 20 \div 3 / 20$, one solver provided the protocol:

$$
4 / 20 \div 3 / 20=4 / 20 \times 20 / 30=4 / 30
$$

The solver inverted the second fraction, but incorrectly wrote the inverse fraction of as 20/30 instead of 20/3. The solver appeared to be cued by the component 20 previously provided in the protocol (i.e., the denominator of the fractions in the problem). Despite applying the algorithm correctly, the solver's solution 4/30 was an error.

Computation errors. Participants made a variety of computation errors. For example, one solver provided the protocol:

$$
4 / 20 \div 3 / 20=4 / 20 \times 20 / 3=40 / 60
$$

This solver correctly inverted the second fraction, correctly multiplied the denominators, $20 \times 3$ to obtain the solution 60 , but then incorrectly multiplied the 
numerators, $4 \times 20=40$. This error is categorized as a computation error because the solver was able to provided correct solutions for other similar problems that involved multiplication involving the operand 20 and was not classified as number substitution error because the component 40 had not previously been provided in the protocol.

Unique errors. Other solvers made strategy errors that were unique to specific problems. For example, one solver performed separate operations on both numerators and denominators. For example, when a solver was presented with the problem $3 / 15 \div$ $2 / 15$, the solver provided the protocol:

$$
3 / 15 \div 2 / 15=(3-2) /(15+15)=1 / 30
$$

The solver subtracted the numerators $3-2=1$ and added the denominators $15+15=30$ to obtain the solution $1 / 30$. However, the solver did not select this strategy for the other division problems.

Solvers also made hybrid errors. These solvers selected the wrong operation or wrong inversion strategy and also made a computation error. For example, when presented with the problem $4 / 20 \div 3 / 20$, one solver provided the protocol:

$$
4 / 20 \div 3 / 20=3.3 / 20
$$

This solver attempted to apply the wrong fraction operation strategy, but further made a computation error when dividing the denominators $4 \div 3=3.3$.

Summary for division. In summary, $30.5 \%$ of 59 solvers made an error on at least one of the division problems. Solvers made systematic strategy errors such as the wrong fraction operation and wrong inversion error. They also made computational errors, number and operation substitution errors, or made combinations of errors.

\section{Discussion}

When solvers performed fraction arithmetic with the aid of written protocols, they made more errors on multiplication and division problems than on addition and 
subtraction. This pattern of results is similar to solvers' difficulties in the online study (Study 1) where solvers made the most errors on division. However, in the current study, multiplication was also more error prone than addition and subtraction because the problems had large operands which participants found difficult to multiply correctly. Thus, arithmetic skill influenced performance more for Study 2 than for Study 1.

Solvers were more likely to use reduction on division than on multiplication problems. Reduction for division was cued because, after inversion of same denominator problems, reduction involved cancelling or dividing the same number from the top and bottom of the new problem for same denominator problems. For different denominator problems, inverting the second fraction focused solvers attention on the second fraction. After inversion, solvers also noted that both numerator and denominator shared a common factor, which also cued the reduction procedure. In contrast, reduction on multiplication problems always involved finding a common factor (see equation (1) below) which presumably takes more effort that noticing the identity relation in same denominator division (see equation (2)) or different denominator division (See equation (3)). Furthermore, even with reduction, the computation required to produce the answer is more difficult for multiplication ( 5 $\times 15)$; after reduction no further calculation is required for division.

(1) $2 / 15 \times 3 / 15=2 \times 3 / 15 \times 15=2 \times 1 / 5 \times 15=2 / 75$

(2) $2 / 15 \div 3 / 15=2 / 15 \times 15 / 3=2 \times 15 / 3 \times 15=2 / 3$

(3) $3 / 4 \div 5 / 10=3 / 4 \times 10 / 5=3 / 4 \times 2=6 / 5$

Because the problems were not equated for difficulty across multiplication and division, it is important to not generalize across operations. 


\section{Problem Characteristics and Solver Profiles that Facilitate Reduction}

A fraction with a numerator-denominator pair that shares a common factor or where common factors are present across operands is a prerequisite for reduction. When presented with reducible problems, solvers were able to identify common factor numerator-denominator pairs and reduced fractions accordingly. There was evidence that operation and denominator-relationship facilitated the reduction procedure. Solvers reduced problems most frequently for division compared to the other operations. Solvers also reduced solutions for different denominator problems more frequently than for same denominator problems, but only for addition and subtraction, suggesting that solvers used their fraction knowledge to decide when to reduce problems appropriately. Solvers' fraction knowledge did not influence selection of the reduction algorithm. Both conceptually- and procedurally-stronger solvers reduced fractions with about equal frequency.

\section{The Source of Errors in Fraction Arithmetic}

Analysis of solvers' protocols provided insight into the variability of their errors. Strategy errors such as the independent whole number, wrong fraction operation, and wrong inversion strategies that observed in the online study were also observed in protocols. However, other errors were also observed, in part, because the problems in Study 2 were more difficult than in Study 1. I characterized these errors as (a) computation, (b) number substitution, (c) operation-substitution, and (d) other errors. Computation errors involved solvers retrieving an incorrect answer for a basic arithmetic operation. Number substitution involved solvers incorrectly using numbers that they had generated in a previous step. Operation substitution involved solvers performing another arithmetic operation instead of the required operation. Combination errors involved solvers selecting the wrong strategy, but also making a 
computational, number-substitution, or operation-substitution error. These errors were not systematic in that the errors were observed for only one problem but not other similar problems for the same participant.

Solvers used protocols as an aid to help them maintain and manipulate information required to solve problems. The use of written protocols also eased the load on working memory during problem solving. For example, dividing fractions required solvers to perform three steps (inverting the second fraction, followed by multiplying numerators and denominators). In the online study, participants may have used written aids, or they may have stored intermediate results in working memory. In the current study, participants were able to write down intermediate products and were encouraged to show all of the steps.

Interestingly, worked protocols occasionally hindered solvers, who used irrelevant information generated at an earlier step resulting in number substitution errors. Both number substitution and computation errors could be traced to limitations of working memory or cognitive resources as solvers performed fraction arithmetic. These errors arose not because solvers were unable to perform the required operation or the computation, but due to constraints on cognitive resources that were available to solvers for solving problems. For example, one solver omitted a digit when multiplying $4 \times 5$ in the protocol (i.e., wrote down 2 instead of 20) and then used the wrong component 2 to calculate the answer. These errors provide support for the relationship between cognitive processes (i.e., attention and working memory) and fraction arithmetic performance reported in other studies (Fuchs, Compton, Fuchs et al., 2012; Fuchs, Geary, Compton, Fuchs, Hamlett, Seethaler, et al., 2010; Geary, Hoard, Byrd-Craven, Nugent, \& Numtee, 2007). In the present study I can only infer that working memory load was the underlying source of these errors. 


\section{Fraction Multiplication and Reduction}

Solvers also experienced greater difficulty when multiplying same- than different-denominator fractions. This effect occurred because the problems were not balanced across denominator relation. Same denominator problems involved multiplying two double-digit denominators (i.e., $3 / 15 \times 2 / 15$ ), whereas different denominator problems required solvers to multiply a single digit and a double-digit denominator (i.e., $2 / 3 \times 2 / 15$ ). Solvers, especially the procedurally-stronger group, experienced difficulty when multiplying fractions with a common denominator.

In summary, there was a relation between solvers' use of reduction and their performance when multiplying fractions. On same-denominator multiplication problems, all of which required multiplication of two-digit numbers, participants did not always use reduction to simplify the calculations. Because solvers attempted to solve a more complex problem with larger components rather than a simpler reduced version with smaller components, they frequently made errors even though every problem had common factors and thus could have been reduced.

When multiplying and dividing fractions, solvers used reduction either before they applied the algorithm for the required operation (i.e., reduction-before-solution), or after they applied the algorithm (i.e., reduction-after-solution). Reduction is never necessary and thus solvers can choose if, and when, to apply reduction. Solvers' selection of the reduction-before-solution strategy indicates that solvers simplify problems to reduce the amount of cognitive effort to solve problems. In contrast, solvers' selection of the reduction-after-solution strategy may be motivated by a learned rule that indicates solutions should be provided in the most-reduced form.

Selection of the reduction step during the fraction arithmetic problem process is of both theoretical and practical interest. Identifying when solvers reduce fractions 
is of theoretical interest because when solvers are required to solve problems, they need to understand that reducing problems is useful for obtaining a correct solution by simplifying the problem, but that simplifying a correct solution is a redundant step. Identifying when solvers reduce fractions is of practical interest because reduction simplifies a more complex problem with large components into a simpler problem with smaller components. Reducing a problem thus enables solvers to apply computational steps on fractions with small components to obtain solutions, whereas not reducing a problem requires solvers to apply computational steps on larger components. The simplest form of a fraction is often the most useful one, for example in algebra problems. Thus, reduction is often a desirable step in fraction problem solving. The hypothesis that accuracy for fraction multiplication decreases when solvers do not reduce fractions will be further tested in Chapter 6 . 


\section{CHAPTER 6: LATENCIES AND EYE TRAJECTORIES}

To date, there has been no empirical research that shows that reducible fraction multiplication problems are solved more accurately and efficiently than nonreducible problems. In Part II of Study 2, I addressed the following questions: "Are reducible fraction multiplication problems solved more accurately and quickly than non-reducible problems?", and "Where do solvers focus their attention when they multiply fractions?"

\section{Are Reducible Fraction Multiplication Problems Solved More Quickly and Accurately Than Non-Reducible Problems?}

For fraction multiplication, the correct solution can be obtained by a two-step algorithm - multiplying the numerators and the denominators. Reducing a numeratordenominator pair in a fraction multiplication problem, that is, using the reduction procedure, adds a third step to the two-step algorithm. People can apply reduction to problems that have common factors - problems without any common factors are nonreducible. There are two types of problems where the reduction procedure is useful. I use the term unit-reducible for problems where the common factors occur in the numerator-denominator pair of the same fraction (i.e., $3 / 5 \times \mathbf{4} / \mathbf{8}$ ). I use the term crossreducible when the common factors occur in the numerator and denominator of different fractions (i.e., $4 / 5 \times 3 / 8$ ). Reduction is optional but it simplifies the numbers in the revised problem, potentially making the computations less difficult. Furthermore, when solvers encounter problems that are too difficult (i.e., the multiplication required is complicated) to solve using the two-step algorithm (e.g., $14 / 15 \times 10 / 21)$, reduction could facilitate obtaining the correct solution.

Reduction, because it adds an extra step, may increase time spent on solving problems. Conversely, reducing fractions to obtain simpler numerators and 
denominator may also result in simpler calculations. Research on multiplication shows that people are slower on problems with larger operands than on those with smaller operands (Campbell \& Graham, 1985; Zbrodoff \& Logan, 1985). These operand-size effects influenced performance in the written protocol portion (Part 1) of Study 2 with multi- digit numbers. Operand size may be even more of an issue in Part II because participants had to do all calculations mentally. Accordingly, I predicted that reduction of problems resulting in multiplication of simpler numerators and denominators should be easier and solved more quickly than those same problems that were not reduced.

There was no evidence that reduction was applied differently across solver groups when multiplying fractions in both the online study (Chapter 4) and when providing worked protocols (Chapter 5). However, there were some differences in the use of reduction across solver groups for division. In Chapter 4, procedurally-stronger solvers were more likely to reduce fractions than conceptually-stronger solvers. In Chapter 5, however, conceptually-stronger solvers were more likely to reduce solutions than procedurally-stronger solvers, but only when dividing fractions. This contradiction in patterns of reduction use across studies can be attributed to the different levels of overall fraction knowledge of participants in each study.

In Chapter 4, participants' procedural scores were higher for conceptuallythan for procedurally-stronger solvers. Conceptually-stronger solvers were more likely to reduce solutions than procedurally-stronger solvers when dividing fractions. In Chapter 5, participants' procedural scores were similar for conceptually- and procedurally-stronger solvers. Thus, because data for the analysis were collected from participants who completed the worked protocols (the participants described in Chapter 5), I focused mainly on the pattern of reduction use from the worked protocol 
findings; and because problems presented to participants were multiplication problems, it would have been difficult to detect differences in reduction use in Chapter 5 .

One reason for the lack group differences in reduction when multiplying fractions might be because solvers used protocols as an aid to multiply multi-digit numbers. If solvers solved complex multiplication problems by directly applying the multiplication algorithm, the worked protocols eliminated the need to use reduction as means of simplifying the calculation. In the second part of Study 2, however, solvers were asked to solve problems without any aids, thus requiring them to use their working memory resources to maintain intermediate steps. Accordingly, I predicted that solvers would use the reduction procedure to simplify problems in Part 2 to reduce the effort required to maintain information in working memory. If conceptually-stronger solvers are indeed more proficient at using reduction than procedurally-stronger solvers, I expected to see a greater frequency of correctly reduced answers for conceptually- than procedurally-stronger solvers. This hypothesis will be tested in both the non-eye-tracking and eye-tracking cohorts.

\section{Where Do Solvers Focus Their Attention When They Multiply Fractions?}

Ocular measures have been used to examine how solvers compare fractions (Huber, Moeller \& Nuerk, 2014; Ischebeck, Weilharter, \& Körner, 2016). For example, Ischebeck et al. compared participants' gaze trajectories in a fraction comparison task (e.g., which is larger $6 / 8$ or $7 / 9$ ?). A gaze trajectory was defined by the pattern of eye fixations, for example, looking back and forth between numerators. When denominators were identical (e.g., 4/8 vs. 5/8), solvers looked back and forth between on numerators. In contrast, when numerators were identical (e.g., 4/9 vs. 4/8), solvers' most frequent gaze trajectories were on denominators. I extended the 
use of gaze trajectories to fraction arithmetic, in order to draw inferences about solvers' strategies when they were multiplying fractions.

When solvers multiply fractions, eye trajectories can be used to determine solution processes. Horizontal (left-to-right) trajectories suggest multiplication of numerators or denominators. Unit-reduction procedures can be inferred from vertical (up-down) eye sequences, whereas cross-reduction procedures can be inferred from diagonal trajectories (See Figure 6.1 and Table 6.1). Trajectory pairs or fixation dyads indicate which fraction component pairs that solvers attend to as they solve the problem and thus provide information about their attempts to extract relevant information.

Figure 6.1. Pairs of fixations on fraction components that correspond to identified algorithmic steps for fraction multiplication.

(a)

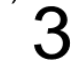

(b) (d)

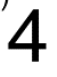

(c) $\times$

${ }^{(e)} 8$

Notes. "a" and "b" represent the numerator and denominator of the left fraction. "d" and "e" represent the numerator and denominator of the right fraction. 
Table 6.1. Pairs of fixations upon fraction components that correspond to identified algorithmic steps for fraction multiplication problems.

\begin{tabular}{lc}
\hline Algorithmic steps (directional dyad) & Corresponding \\
& Eye trajectories \\
\hline Numerator Multiplication (horizontal) & $\mathrm{ad} / \mathrm{da}$ \\
Denominator multiplication (horizontal) & $\mathrm{be} / \mathrm{eb}$ \\
Left fraction unit-reduction (vertical) & $\mathrm{ab} / \mathrm{ba}$ \\
Right fraction unit-reduction (vertical) & $\mathrm{de} / \mathrm{ed}$ \\
Left numerator-right denominator cross-reduction (diagonal) & $\mathrm{ae} / \mathrm{ea}$ \\
Right numerator-left denominator cross-reduction (diagonal) & $\mathrm{bd} / \mathrm{db}$
\end{tabular}

Reduction can occur either on the same unit pair or across pairs. I predicted that the vertical fixation dyad count will be higher on unit-reducible than on crossreducible and non-reducible problems. Cross-reduction involves identifying a common factor in numerator-denominator pairs from different fractions. Thus, I predict the diagonal fixation dyad count will be higher on cross-reducible than on unit-reducible or non-reducible problems. This hypothesis will be tested only for the eye-tracking cohort.

\section{Hypotheses}

To summarize, Part II of Study 2 test the following hypothesis:

1. Accuracy will be higher for unit-reducible and cross-reducible than nonreducible problems.

2. Conceptually-stronger solvers are more likely to select and apply reduction than procedurally-stronger solvers.

3. Latencies will be shorter for unit-reducible and cross-reducible than non- 
reducible problems when participants use reduction.

4. There will be more vertical dyads for unit-reducible than for crossreducible and non-reducible problems.

5. There will be more diagonal dyads for cross-reducible than unit-reducible and non-reducible problems.

\section{Method}

The 59 participants who participated in the first part of Study 2 also participated in the second part. The participants completed the same set of problems, as described below. The procedures were the same, except that for participants in the eye-tracking cohort, eye-tracking data was also collected. Thus, the behavioural data are analyzed for all 59 participants whereas eye tracking data was only available for 32 participants.

\section{Participants}

Twenty-seven participants were randomly assigned to the non eye-tracking cohort and thirty-two participants were randomly assigned to the eye-tracking cohort.

\section{Materials}

Fraction arithmetic problems. The 30 problems shown in Table 6.2 , and the converse fractions formed by switching the position of the fractions for each problem were presented to participants in the second part of Study 2 for a total of 60 multiplication problems. Unit-reducible problems consisted of one fully reduced fraction (e.g., 3/5) and one fraction that had common factors in the numerator and denominator and thus could be reduced (e.g., 4/8). Cross-reducible problems were constructed by switching the numerators for the reducible fraction multiplication problems (e.g., changing $3 / 5 \times 4 / 8$ to $4 / 5 \times 3 / 8$ ). Non-reducible problems consisted of two fractions without reducible or cross-reducible numerator-denominator pairs (e.g., 
$3 / 5 \times 4 / 7)$. Non-reducible fraction multiplication problems were introduced to assess whether there are differences in solution time or accuracy between reducible and nonreducible problems. Non-reducible problems were formed by either increasing or decreasing the denominator of the unit-reducible problems by 1 or 2 . Thus, $3 / 4 \times 7 / 35$ was transformed to $3 / 4 \times 7 / 37$.

Table 6.2. Unit-reducible, cross-reducible, and non-reducible multiplications problems used in the second part of Study 2.

\section{Fraction multiplication problems}

\begin{tabular}{ccc} 
Unit-reducible & Cross-reducible & Non-reducible \\
$3 / 5 \times 4 / 8=12 / 40$ & $4 / 5 \times 3 / 8=12 / 40$ & $3 / 5 \times 4 / 7=12 / 35$ \\
$3 / 5 \times 2 / 8=6 / 40$ & $2 / 5 \times 3 / 8=6 / 40$ & $3 / 5 \times 2 / 7=6 / 35$ \\
$2 / 5 \times 3 / 9=6 / 45$ & $3 / 5 \times 2 / 9=6 / 45$ & $2 / 5 \times 4 / 11=8 / 55$ \\
$3 / 5 \times 7 / 14=21 / 70$ & $7 / 5 \times 3 / 14=21 / 70$ & $3 / 5 \times 7 / 13=21 / 65$ \\
$2 / 3 \times 5 / 25=10 / 75$ & $5 / 3 \times 2 / 25=10 / 75$ & $2 / 3 \times 5 / 27=10 / 81$ \\
$3 / 4 \times 5 / 25=15 / 100$ & $5 / 4 \times 3 / 25=15 / 100$ & $3 / 4 \times 5 / 23=15 / 92$ \\
$3 / 5 \times 4 / 20=12 / 100$ & $4 / 5 \times 3 / 20=12 / 100$ & $3 / 5 \times 4 / 19=12 / 95$ \\
$2 / 5 \times 7 / 21=14 / 105$ & $7 / 5 \times 2 / 21=14 / 105$ & $2 / 5 \times 7 / 19=14 / 95$ \\
$2 / 3 \times 7 / 35=14 / 105$ & $7 / 3 \times 2 / 35=14 / 105$ & $2 / 3 \times 7 / 33=14 / 99$ \\
$3 / 4 \times 7 / 35=21 / 140$ & $7 / 4 \times 3 / 35=21 / 140$ & $3 / 4 \times 7 / 37=21 / 148$ \\
\hline
\end{tabular}

I constructed problems to have similar computational demands across the three problem types. The average product of the numerators was 13.1 for all three groups. The average product of the denominators was 82,82 , and 80 for unit-reducible, crossreducible, and non-reducible problems, respectively. A paired-sample t-test comparing the solutions of denominators for unit-reducible problems with the 
corresponding non-reducible problems revealed no differences in denominator size, $t(9)=0.10, p=.93$. Note that denominators for both the unit-reducible and crossreducible problems were identical because of how the problems were created, thus the variance of denominator size across these two problem groups were identical. However, because of the method used in creating the problems, all unit- and crossreducible problems were multiples of 5 , whereas only $60 \%$ of the problems were multiples of 5 .

The practice block consisted of the 12 different-denominator reducible fraction multiplication problems that were presented to participants in Part I of Study 2.

\section{Procedure}

Participants were seated approximately $60 \mathrm{~cm}$ in front of a desktop computer with a 15 -inch by 13 -inch monitor. Participants then followed the procedure for either the first cohort (eye-tracking data was not collected) and the second cohort (eyetracking data was collected). At the start of the study, participants were first presented with a practice block of 12 problems, followed by three experimental blocks of 20 problems each. Participants were allowed to take a break after each block.

Non eye-tracking cohort. Each problem was initiated by the participant first pressing the space bar. A fixation point then appeared at the centre of the screen and remained on the screen for $500 \mathrm{~ms}$ followed by a fraction arithmetic problem. There was no delay between the offset of the fixation point and the onset of the problem. The operation symbol " $\times$ " appeared at the same location as the fixation point. Participants were asked to verbally state their answer into a microphone placed directly in front of them and their responses were connected to an ASIO voice trigger accurate to $+/-1 \mathrm{~ms}$. Participants were told to answer as quickly and accurately as possible. They were also told not to speak until they were sure of their answer. This 
instruction was to ensure that solvers did not immediately multiply numerators, then pause while they multiplied the denominators, for example. If participants asked whether they were required to reduce solutions, they were told "it is up to you". As soon as the microphone detected a response, the problem disappeared. and the researcher recorded the response using a keyboard on a separate computer. The time taken to solve each problem was the time from the onset of each problem to the onset of the time taken by the microphone to detect a response. The screen remained blank until the participant initiated the next trial. See Figure 6.2 for a visual presentation and corresponding timeframe of a typical problem and the corresponding response to the problem.

Figure 6.2. Outline of the timeframe of a typical problem in the non eye-tracking cohort of Study 2. Participant initiates a trial by pressing the space bar: (a) Fixation point appears for $500 \mathrm{~ms}$, (b) offset of fixation point and onset of problem, (c) Problem remains on screen until microphone detects participant's voice.

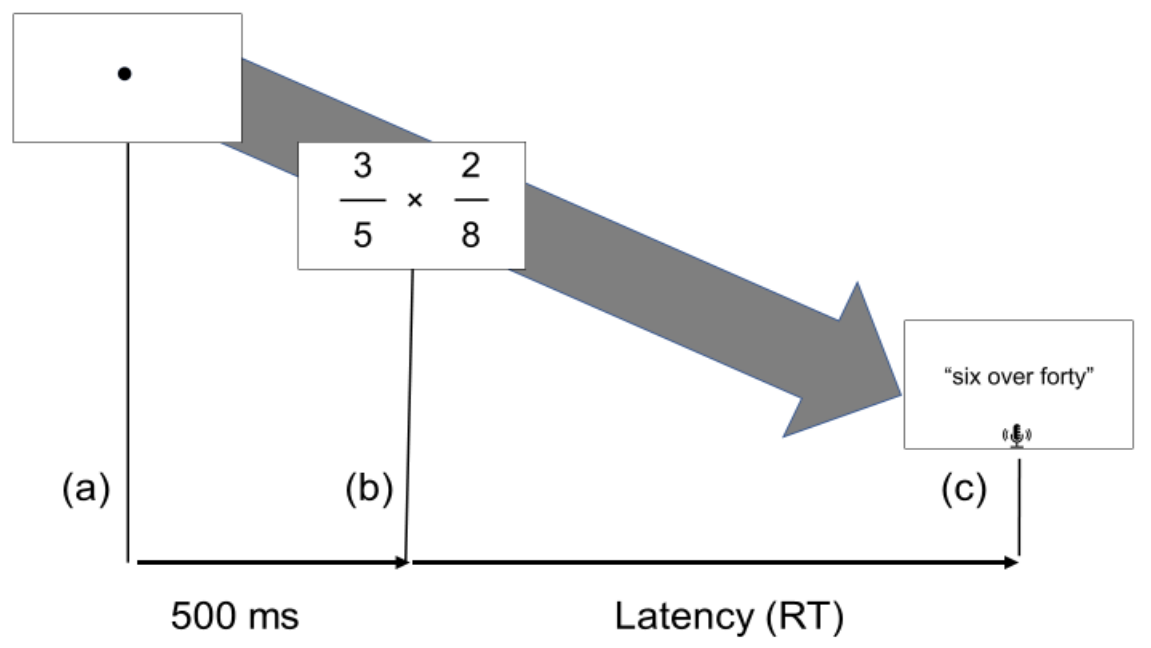

Eye-tracking cohort. Problems were presented on the computer connected to an SR-Eyelink eye tracker. Participants were required to lean their forehead against the forehead bar and rest their chin upon a chin rest. This procedure was to ensure that participants' eyes were kept in a constant position for accurate recording of the gaze 
locations. The practice block and the three experimental blocks started with a calibration phase to ensure accurate recording of ocular measures by the eye-tracker.

Each problem was initiated by the participant first pressing the space bar.

A $1 \times 1.5 \mathrm{~cm}$ rectangle randomly appeared at one of the four corners of the screen. Participants were required to fixate upon the rectangle. Upon the recording of an eye fixation on the rectangle, the fraction arithmetic problem appeared. The requirement that participants fixate their eyes on the rectangle ensured that their attention would not be fixated upon areas of the screen where the problem would appear, which could produce artificial fixations and inflation of dwell times on the problem. Otherwise the instructions and procedure were the same as cohort 1. See Figure 6.3 for a visual presentation and corresponding timeframe of a typical problem and the corresponding response to the problem.

Figure 6.3. Outline of the timeframe of a typical problem in the eye-tracking cohort of Study 2. Participant initiates a trial by pressing the space bar: (a) rectangular box appears in one corner of the screen and remains until the participant fixates on the box, (b) offset of fixation point and onset of problem, (c) problem remains on screen until microphone records participant's voice.

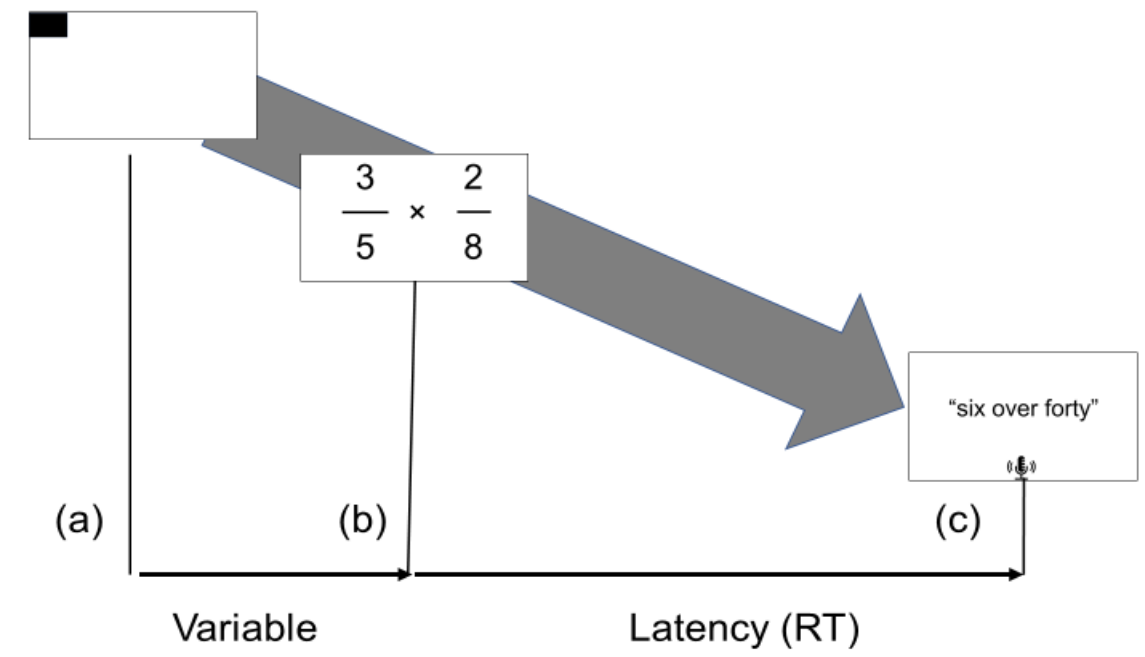

To analyze the eye tracking data, five interest areas were defined, one around 
each problem element. Each interest area measured $6.7 \mathrm{~cm}$ by $6.7 \mathrm{~cm}$ and was centered on the corresponding problem element (i.e., the operation sign and the four digits). The borders of each interest area were connected but did not overlap. Fixations upon each problem element were defined as fixations within the corresponding interest area for that element (see Figure 6.4).

Figure 6.4. Dimensions of interest regions centered on problem elements for a typical problem in the eye-tracking cohort. The dimension of each interest area is $6.7 \mathrm{~cm} \times$ $6.7 \mathrm{~cm}$. Participants did not see the lines; they are shown here to indicate the interest areas. Fixations outside of these interest areas were not analyzed.

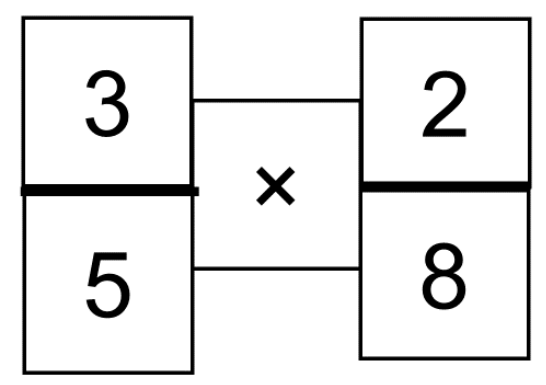

\section{Results}

Of the 59 participants who completed Study 2, data from three participants were excluded because of the failure of the voice recognition software to identify the onset of participants' voices. Demographics of the remaining 56 participants are shown in Table 6.3. There were about an equal amount of procedurally- and conceptually-stronger solvers in each study. There were more conceptually-stronger male than female solvers in the eye-tracking cohort. This unequal sex distribution reflected the random assignment of participants to each study. Sex distribution among the other combinations of study and solver profile were about equally distributed. 
Table 6.3. Characteristics of participants in the eye-tracking and non-eye-tracking cohort. Median age in years.

\begin{tabular}{|c|c|c|c|c|c|c|}
\hline \multirow[t]{2}{*}{ Cohort } & \multicolumn{3}{|c|}{$\begin{array}{l}\text { Procedurally-stronger solvers } \\
\qquad(\mathrm{n}=30)\end{array}$} & \multicolumn{3}{|c|}{$\begin{array}{l}\text { Conceptually-stronger solvers } \\
\qquad(\mathrm{n}=26)\end{array}$} \\
\hline & $N$ & $\%$ female & $\begin{array}{l}\text { Median } \\
\text { age }\end{array}$ & $n$ & $\begin{array}{c}\% \\
\text { female }\end{array}$ & $\begin{array}{l}\text { Median } \\
\text { age }\end{array}$ \\
\hline $\begin{array}{l}\text { Non eye- } \\
\text { tracking }\end{array}$ & 14 & 64.2 & 20 & 14 & 50.0 & 20 \\
\hline $\begin{array}{l}\text { Eye- } \\
\text { tracking }\end{array}$ & 16 & 62.5 & 19 & 12 & 16.7 & 19 \\
\hline
\end{tabular}

\section{Problem Solving Performance}

In this section I analyze accuracy (i.e., whether the problem solution was correct), use of the reduction procedure, and latencies on correct trials. These analyses were done on all of the participants in both cohorts. In the following section, I analyze eye trajectories for the participants in the eye-tracking cohort.

Accuracy. Participants' responses were coded as "correct" if the response had the same value as the correct answer, regardless of whether reduction was used. The percentage of correctly solved problems for each problem type (unit-reducible vs. cross-reducible vs. non-reducible) was calculated as the percentage of correct responses divided by the number of problems in each problem type $(n=20)$. Percentage correct was analyzed in a 2 (solver group: procedurally-stronger vs. conceptually-stronger) by 2 (Cohort: non eye-tracking vs. eye-tracking) by 3 (problem type: unit-reducible vs. cross reducible vs. non-reducible) mixed ANOVA. Post-hoc comparisons were tested using Tukey HSD at .05 level of significance. There was a main effect of problem type, $F(2,51)=3.91, p=0.03, \eta^{2}=.13$. Solvers 
were less accurate on non-reducible (75.6\%) problems than on unit-reducible $(80.1 \%)$ and cross-reducible $(80.2 \%)$ problems, $p \mathrm{~s}<.05$. Accuracy was not significantly different for procedurally- and conceptually-stronger solvers $(77.4 \%$ vs. $79.9 \%), F(1$, $52)=0.14, p=0.71$, nor did it differ across cohorts $(80.6 \%$ vs. $76.6 \%$, for non eyetracking vs. eye-tracking), $F(1,52)=0.36, p=0.55$. There was, however, a significant interaction between solver group and problem type, $F(2,51)=3.39, p=$ $0.04, \eta^{2}=.12$, as shown in Figure 6.5

Conceptually-stronger solvers performed slightly better than procedurallystronger solvers on unit-reducible and non-reducible problems. However, the differences were not significant. There were no differences for cross-reducible problems across both solver groups and no other interactions. These differences reflected a larger variability in accuracy for unit-reducible and non-reducible problems across both groups of solvers, but not for cross-reducible problems Figure 6.5. Percentage accuracy scores obtained by solver group (procedurally- and conceptually-strong solvers) and problem type (unit-reducible vs. cross-reducible vs. non-reducible). Error bars are 95\% CI calculated according to Jarmasz and Hollands (2009).

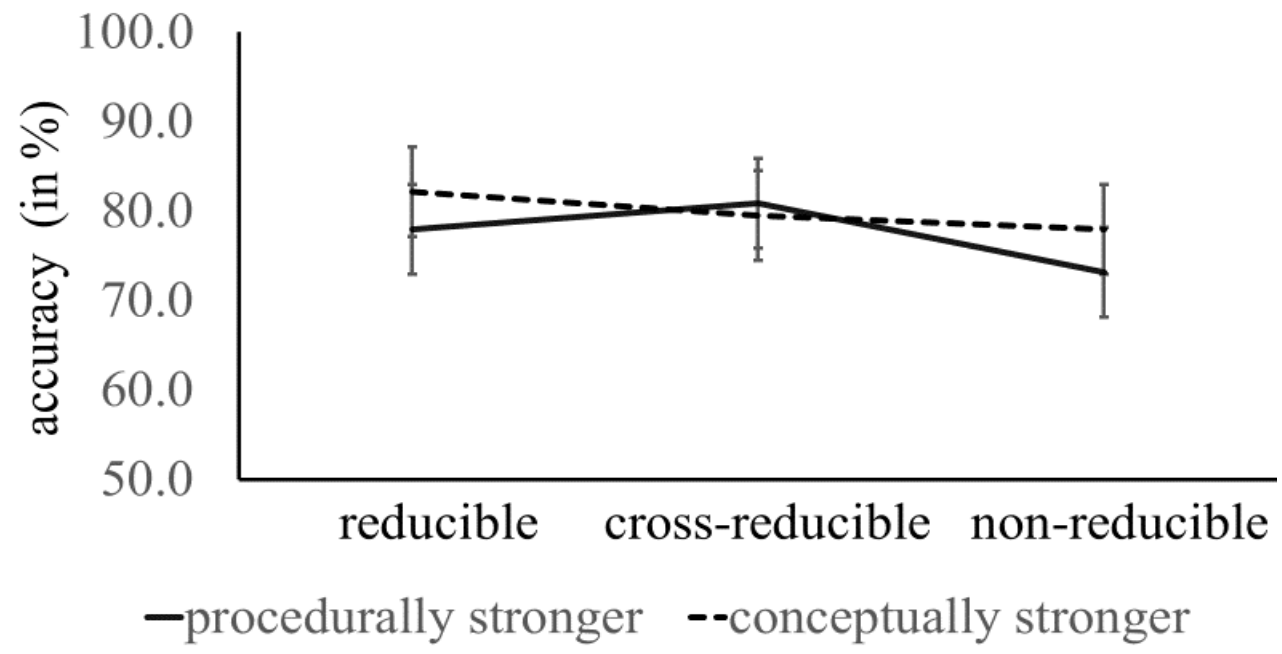

Use of reduction. Frequencies of correctly reduced answers for unit-reducible 
and cross-reducible problems for each participant were expressed as percentage scores calculated from correctly reduced answers over the number of each type of problem within each condition $(n=20)$. Many participants did not use reduction at all, resulting in a large number of zero values in the analysis for accuracy and latencies for correctly-reduced solutions. Mean percentages of answers that were correct and reduced were analyzed in a 2 (solver group: procedurally-stronger vs. conceptuallystronger) by 2 (problem type: reducible vs. cross reducible) mixed ANOVA.

I predicted that people in the procedurally-stronger group would use reduction less than people in the conceptually-stronger group. Although the difference was not significant, the direction of the effect was consistent with this prediction procedurally-stronger participants used reduction on $9.4 \%$ of correct trials whereas conceptually-stronger participants used reduction on $20.9 \%$ of correct trials, $F(1,54)$ $=2.02, p=0.16$. None of the other main effects or interactions were significant. On average, reduced solutions were provided on $15.7 \%$ of the reducible problems and $15.9 \%$ of the cross-reducible problems. Thus, the location of the numbers that shared common factors did not influence use of reduction in this problem set.

To further compare whether conceptually-stronger solvers used reduction more frequently than procedurally-stronger solvers, I determined whether each solver provided a correctly reduced answer for at least one problem. Fifteen participants (26.7\% of all participants) provided a reduced solution on at least one problem. Of these participants, five $(20 \%)$ were procedurally-stronger solvers and ten $(38.5 \%)$ were conceptually-stronger solvers. A $\chi^{2}$ test of association revealed that more conceptually-stronger solvers $(62.5 \%)$ provided at least one correctly reduced answer than procedurally-stronger solvers $(25 \%), \chi^{2}(1, N=56)=14.72, p<.001, \varphi=0.25$. On average, these 15 participants used reduction on $55 \%$ of the unit-reducible and 
cross-reducible problems. The percentage use of reduction for unit- and crossreduction problems for the solvers who used reduction at least once was compared in a paired samples t-test. There was no difference across problem type in the use of reduction $($ unit-reducible $=54.0 \%$ vs. cross-reducible $=56.0 \%), t(14)=0.46, p=0.64$.

In summary, solvers could be classified into solvers who reduced at least one problem and non-reducers who did not reduce any problems. Solvers who reduced at least one problem were classified as reducers. In general, they used reduction for about half of the problems. Solvers who did not reduce any problem were classified as non-reducers. Reducers were more likely to be conceptually-stronger than procedurally-stronger solvers and used reduction on about half of the reducible problems. Furthermore, frequency of reduction use was similar for both conceptuallyand procedurally-stronger solvers, suggesting that selection of the reduction procedure was not related to only conceptual or procedural knowledge, but a combination of both types of fraction knowledge.

Latencies. Five participants provided correct answers for less than $50 \%$ of all problems. The average correct percentage of these participants ranged from 0 to $33 \%$. Data for these participants were excluded from the latency analysis. The average correct percentage of the remaining participants ranged from $53.3 \%$ to $100 \%$. Three of these participants were procedurally-stronger solvers and two participants were conceptually-stronger solvers. None of these five participants used reduction. Of the problems attempted by the remaining 51 participants, 468 of 3060 problems $(15.3 \%)$ were answered incorrectly. These problems were excluded from the analysis of latencies. Another 95 problems (3.7\%) were excluded due to the failure of the voice recognition software to detect a response.

For each participant in each condition, median latencies for correct trials were 
calculated. Medians were used instead of means to compensate for some solvers' low accuracy rates within a problem type. Median latencies were analyzed using a 2 (solver group: procedurally-stronger vs. conceptually-stronger) by 2 (Cohort: non eye-tracking vs. eye-tracking) by 3 (problem type: item-reducible vs. cross reducible vs. non-reducible) mixed ANOVA. Post-hoc comparisons were tested using Tukey HSD at .05 level of significance. Figure 6.6 presents means of median latencies of correctly solved problems across solvers.

Figure 6.6: Means of median latencies (in ms) by solver group (procedurally-and conceptually-stronger) and problem type (reducible vs. cross-reducible vs. nonreducible). Error bars are 95\% CIs calculated according to Jarmasz and Hollands (2009).

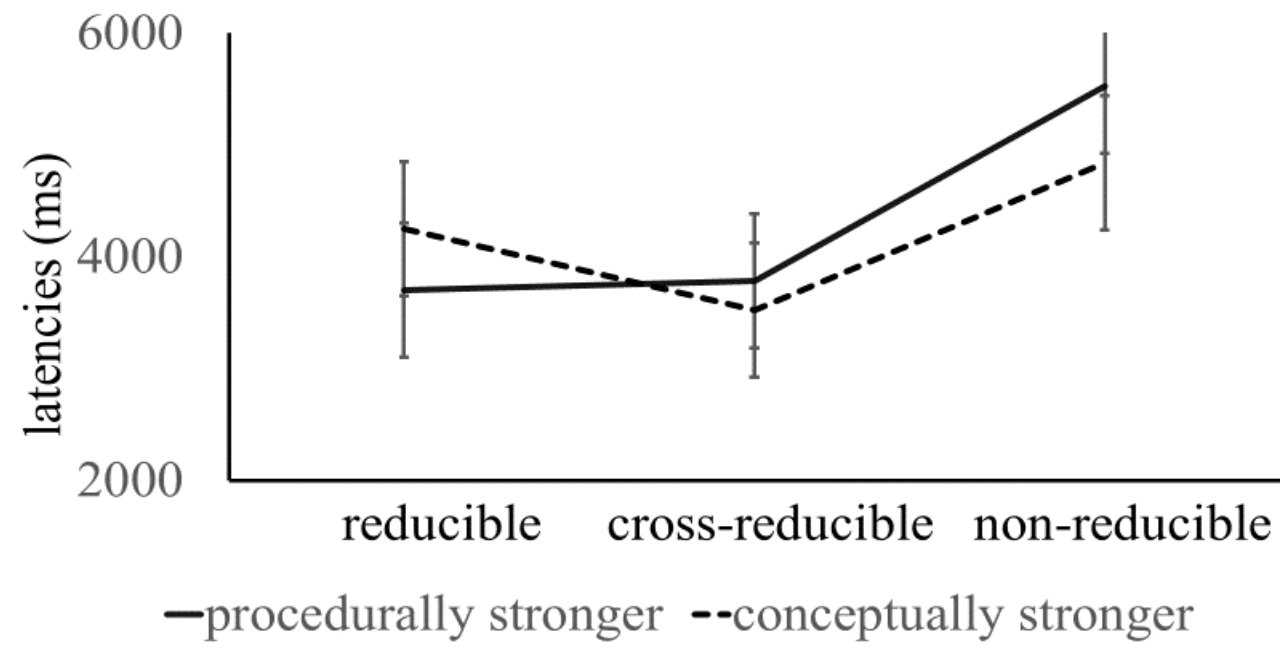

There was a main effect of problem type, $F(2,46)=25.62, p=.001, \eta^{2}=.53$. Participants solved reducible (3980 ms) and cross-reducible problems (3654 ms) faster than non-reducible problems $(5184 \mathrm{~ms}), p s<.05$. However, there were no differences in latencies between procedurally- and conceptually-stronger solvers (4338 vs. $4207 \mathrm{~ms}), F(1,47)=0.03, p=0.87$. Latencies were not significantly different across cohorts (non-eye-tracking=4866 vs. eye-tracking $=3678 \mathrm{~ms}), F(1,47)$ $=2.3, p=.14$. There were no interactions. Comparison of latencies across problem 
type corroborates findings from the accuracy analysis. Solvers were faster and more accurate on unit-reducible and cross-reducible than on non-reducible problems.

To clarify whether observed differences in latencies could be attributed to solvers' use of reduction, latencies were analyzed separately for participants who used reduction versus those who did not in one-way repeated measures ANOVAs by problem group (i.e., unit reducible vs. cross reducible vs. non-reducible).

Figure 6.7: Means of median latencies (in $\mathrm{ms}$ ) for reducers and non-reducers by problem type (unit-reducible vs. cross-reducible vs. non-reducible) problems. Error bars are 95\% CI calculated according to Jarmasz and Hollands (2009).

Non-reducers

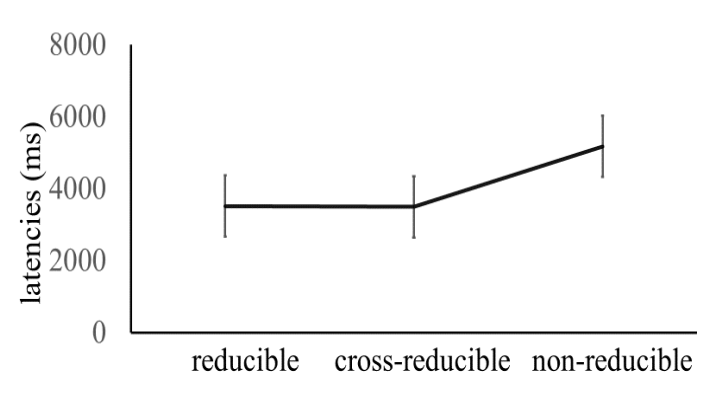

Reducers

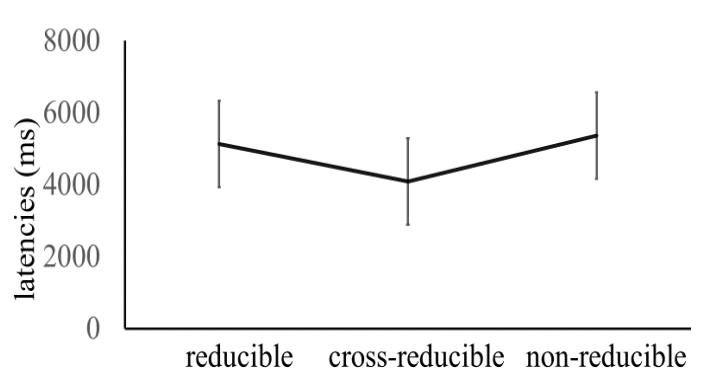

As shown in the left panel of Figure 6.7, people who did not use reduction (i.e., non-reducers) solved unit-reducible and cross-reducible problems (3422 and $3500 \mathrm{~ms}$ ) faster than non-reducible problems $(5175 \mathrm{~ms}), F(2,34)=20.71, p<0.001$, $\eta^{2}=.38$. In contrast, people who used reduction solved cross-reducible problems faster than non-reducible problems (4086 vs. 5373), $F(2,13)=6.39, p<0.01, \eta^{2}=$ .50 , whereas unit-reducible problems were not different from either of these problem types $(5139 \mathrm{~ms})$. These findings do not support my hypothesis that use of reduction is the source of differences in latency and accuracy (i.e., as shown in Figure 6.7) between the two reducible problem types and the non-reducible problems. 
Fixation Dyads. To determine whether reduction use was related to patterns of eye gaze, I analyzed fixation dyads for the participants who solved problems in the second part of Study 3. Of these 28 participants, four participants correctly answered less than $50 \%$ of all problems. Three of these participants were procedurally-stronger solvers, one participant was a conceptually-stronger solver. Data from these participants were excluded from the analysis for fixation dyads. Of the problems attempted by the remaining 24 participants, 216 problems $(15.0 \%)$ were answered incorrectly. These problems were excluded from the analysis. Another 12 problems $(1.0 \%)$ were excluded due to the failure of the voice recognition software to register a proper response. A total of $16.0 \%$ of the problems were excluded from the trajectory analysis. Of the remaining 24 participants there were 6 reducers $(25 \%)$.

The average number of fixation dyads for each person in each conditions was analyzed in a 2(solver group: procedurally-stronger vs. conceptually-stronger), 3(problem type: reducible vs. cross reducible vs. non-reducible) by 3(dyad type: horizontal vs. vertical vs. diagonal) mixed ANOVA. Post-Hoc comparisons were tested using Tukey HSD at .05 level of significance. Means are shown in Table 6.4. 
Table 6.4. Means (and standard errors) of the number of fixation dyads by solver group (procedurally-stronger vs. conceptually strong solvers), problem type (reducible vs. cross-reducible vs. non-reducible) and fixation dyad type (vertical vs. diagonal vs. horizontal).

\begin{tabular}{lcccc}
\hline \multicolumn{5}{c}{ Procedurally-stronger $(\mathrm{n}=13)$} \\
\hline Reducible & Cross-reducible & Non-reducible & Mean \\
\hline Viagonal & $0.51(0.2)$ & $0.55(0.1)$ & $0.46(0.1)$ & $0.50(0.1)$ \\
Hertical & $1.33(0.2)$ & $1.11(0.2)$ & $1.23(0.2)$ & $1.22(0.2)$ \\
Horizontal & $3.90(0.5)$ & $3.26(0.5)$ & $3.47(0.4)$ & $3.54(0.4)$ \\
\hline Mean & $1.91(0.2)$ & $1.64(0.2)$ & $1.71(0.2)$ \\
\hline \multicolumn{5}{c}{ Conceptually-stronger $(\mathrm{n}=11)$} \\
\hline Diagonal & $0.77(0.2)$ & $0.62(0.1)$ & $0.49(0.1)$ & $0.62(0.1)$ \\
Vertical & $1.41(0.2)$ & $1.08(0.2)$ & $1.02(0.2)$ & $1.17(0.2)$ \\
Horizontal & $3.17(0.6)$ & $2.57(0.5)$ & $2.15(0.4)$ & $2.63(0.5)$ \\
\hline Mean & $1.78(0.2)$ & $1.4(0.2)$ & $1.2(0.2)$ & \\
\hline
\end{tabular}

There was a main effect of dyad type, $F(2,21)=38.85, p<.001, \mathrm{\eta}^{2}=.79$. Solvers made the more horizontal dyads (3.1) than vertical dyads (1.2); diagonal dyads $(0.5)$ were made least frequently, all $p \mathrm{~s}<.05$. There was also a main effect of problem type, $F(2,21)=9.28, p<.001, \eta^{2}=.47$. Overall, solvers made more trajectories on reducible problems (1.8) than on cross-reducible (1.5) or non-reducible problems (1.5), $p s<.05$. On average, conceptually- and procedurally-stronger solvers made the same number of fixation dyads per problem $(1.5$ vs. 1.8$), F(1,22)=0.94, p$ $=0.34$. There was an interaction between solver group and problem type, $F(2,21)=$ 4.19, $p=0.03, \eta^{2}=.29$, as shown in Figure 6.8. Conceptually-stronger solvers made fewer fixation dyads than procedurally-stronger solvers on non-reducible problems (1.2 vs. 1.7), $p<.05$. However, there were no differences in the number of fixation 
dyads between procedurally- and conceptually-stronger solvers for reducible and cross-reducible problems. No other effects were significant.

Figure 6.8: Average number of fixation dyads by dyad type (vertical vs. diagonal vs. horizontal) and solver group (procedurally-and conceptually-stronger solvers.-Error bars are 95\% CI calculated according to Jarmasz and Hollands (2009).

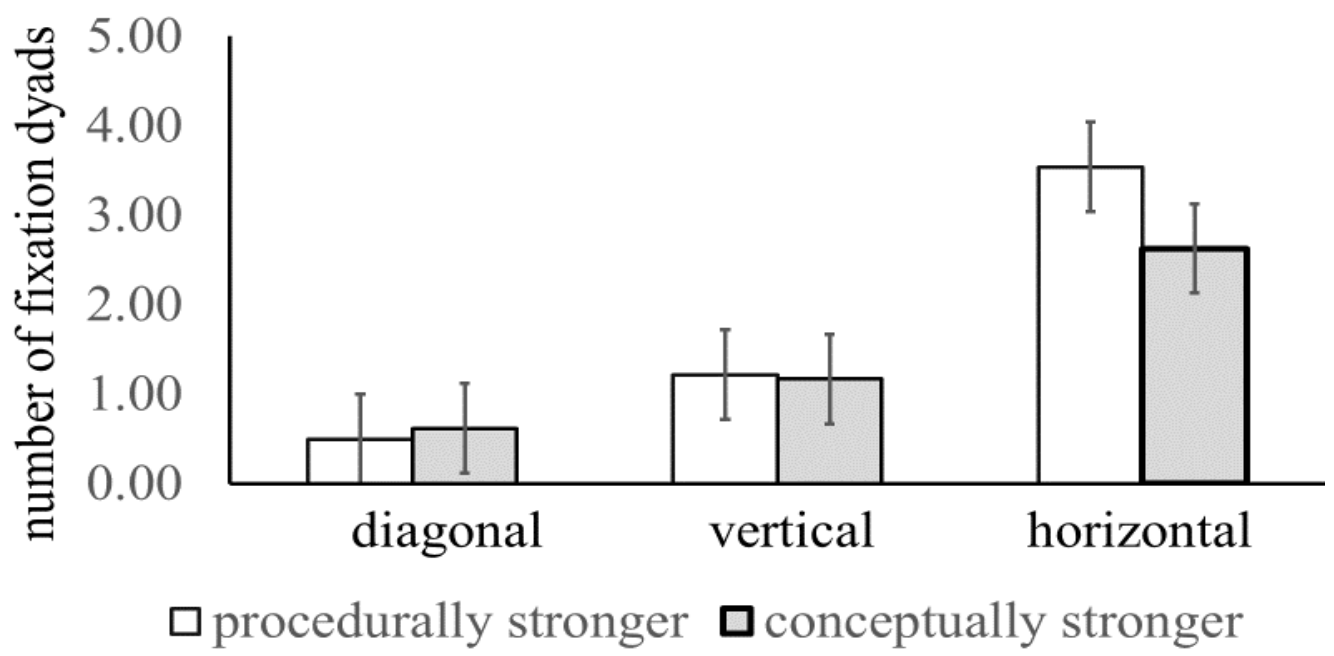

In summary, solvers made the most horizontal trajectories, consistent with the requirements of the two-step multiplication algorithm (i.e., multiply numerators and multiply denominators). I had predicted that solvers would make more vertical trajectories on unit-reducible problems. They made, on average, 1.2 vertical trajectories per problem, but this did not vary across problem types. I had predicted that there would be more diagonal trajectories on cross-reducible problems. Solvers made 0.6 diagonal trajectories per problem and these did not vary across problem type. Finally, conceptually-stronger solvers made fewer horizontal trajectories than procedurally-stronger solvers, suggesting that conceptually-stronger solvers attended to the sequence of components corresponding to numerator and denominator multiplication less frequently than procedurally-stronger solvers.

Analysis of reduction use revealed that some conceptually- and some procedurally-stronger solvers used reduction to solve problems. Thus, analyzing the 
number of fixation dyads across solver groups rather than across reducer groups may have obscured relations between strategy selection and eye trajectories. Thus, I analyzed eye trajectories by whether participants used reduction. Table 6.5 presents means (and standard errors) of the number of fixation dyads by reducer group (reducers vs. non-reducers), problem type (reducible vs. cross-reducible vs. nonreducible) and fixation dyad type (vertical vs. diagonal vs. horizontal).

Table 6.5. Means (and standard errors) of the number of fixation dyads by solver group (procedurally-stronger vs. conceptually strong solvers), problem type (reducible vs. cross-reducible vs. non-reducible) and fixation dyad type (vertical vs. diagonal vs. horizontal).

\begin{tabular}{lcccc}
\hline \multicolumn{5}{c}{ reducers $(\mathrm{n}=6)$} \\
\hline Diagonal & $0.95(0.2)$ & $0.85(0.2)$ & $0.63(0.2)$ & $0.81(0.2)$ \\
Vertical & $1.52(0.3)$ & $1.07(0.3)$ & $1.20(0.3)$ & $1.26(0.2)$ \\
Horizontal & $2.43(0.7)$ & $1.95(0.7)$ & $1.90(0.7)$ & $2.09(0.6)$ \\
\hline Means & $1.92(0.2)$ & $1.62(0.2)$ & $1.57(0.2)$ & \\
\hline & \multicolumn{5}{c}{ non-reducers $(\mathrm{n}=18)$} \\
\hline Diagonal & $0.52(0.1)$ & $0.49(0.1)$ & Non-reducible & Means \\
Vertical & $1.32(0.1)$ & $1.11(0.2)$ & $1.11(0.2)$ & $1.18(0.1)$ \\
Horizontal & $3.94(0.4)$ & $3.28(0.4)$ & $3.18(0.3)$ & $3.47(0.4)$ \\
\hline Means & $1.63(0.3)$ & $1.29(0.3)$ & $1.25(0.3)$ & \\
\hline
\end{tabular}

As shown in Table 6.5, despite the small sample of reducers $(n=6)$, the differences in horizontal trajectories across reducer groups approached significance. Non-reducers made slightly more horizontal trajectories than reducers (3.47 vs. 2.09), $t(22)=1.94, \underline{p}=.07$. In comparison, reducers made slightly more diagonal trajectories than non-reducers $(0.81$ vs. 0.47$), t(22)=1.74, p=.10$. The number of vertical trajectories did not differ across reducers and non-reducers (1.26 vs. 1.18$), t(22)=.31$, 
$p=.76$. This pattern of results suggests that non-reducers focused more of their attention on extracting and using information for steps related to multiplication of numerators and denominators than reducers. Reducers, in contrast, made more diagonal trajectories than non-reducers, perhaps to assess whether the conditions for cross-reduction were satisfied. These conclusions are tentative, given the small number of people who used reduction in the present research.

\section{Discussion}

\section{Are Reducible Fraction Multiplication Problems Solved More Quickly and Accurately Than Non-Reducible Problems?}

In general, reducible and cross-reducible problems were solved more quickly and accurately than non-reducible problems. One interpretation of these findings is that application of the three-step algorithm with reduction was more efficient than application of the two-step algorithm of directly multiplying fractions. However, people who used reduction were not faster or more accurate on reducible than nonreducible problems. Instead, another effect emerged. Non-reducers solved reducible problems more quickly than non-reducible problems. The use of reduction did not reduce solution times. Instead, non-reducers may have been faster overall because they simply applied the two-step algorithm without taking time to decide whether to use reduction. Thus, the present results do not support the view that reduction results in shorter problem solution times.

There were no differences in accuracy and problem-solving time between conceptually- and procedurally-stronger solvers. Although more of the conceptuallystronger solvers reduced at least one problem then the procedurally-stronger solvers, some people in each group of conceptually- and procedurally-stronger solvers could be classified as reducers. Note that solvers were not required to reduce problems as 
part of their instructions. As such, it is possible that some of the procedurally-stronger solvers might have reduced fractions if they were instructed to do so.

The observation that reducers were more likely to be conceptually- than procedurally-stronger solvers, suggests that solvers with a relatively stronger knowledge of concepts than procedures had a tendency to multiply fractions by using reduction to simplify fractions. As a consequence of selecting the reduction procedure, reducers who used the three-step algorithm also took longer to solve problems than solvers who applied the two-step direct multiplication algorithm.

\section{Where Do Solvers Focus Their Attention When They Multiply Fractions?}

Overall, solvers made more horizontal than vertical or diagonal fixation dyads as they multiplied fractions. Solvers' sequential attention to horizontally-aligned numerator and denominator pairs is assumed to reflect the process of attending to and extracting numerical information in preparation for multiplying these fraction components. There were more horizontal than vertical or diagonal trajectories because the multiplication step was necessary to obtain correct solutions for all problems. In contrast, vertical and diagonal dyads were assumed to reflect solvers' attention to numerator-denominator pairs as they checked the conditions for reduction or crossreduction, respectively. There were no differences in the number of vertical and diagonal dyads across solver groups, suggesting that conceptually- and procedurallystronger solvers were comparable in their attentional processes when attempting to identify the conditions for the reduction and cross-reduction algorithm. Reduction and cross-reduction were optional steps that were used infrequently and thus solvers did not expend as much attention checking for the conditions of reduction.

However, a comparison of gaze trajectories between reducers and nonreducers provided a clearer picture of the attentional processes related to selection and 
execution of the reduction strategy. The pattern of gaze trajectories suggests that reducers made more diagonal trajectories and fewer horizontal trajectories than nonreducers. These results should be interpreted cautiously because there were only six reducers in the Study 2 sample. Nevertheless, it is possible to outline a framework of the individual differences in reduction use across both reducer groups. When presented with a problem, reducers searched for common factor numeratordenominator pairs to prepare for reduction. They then extracted information from the reducible pair to reduce numerator-denominator pairs to simpler components, and as a result performed the multiplication algorithm on smaller numerator-numerator and denominator-denominator components, resulting in fewer horizontal trajectories than non-reducers. Non-reducers directly applied the multiplication algorithm on larger numerator-numerator and/or denominator-denominator components, resulting in more horizontal trajectories than reducers.

\section{Limitations}

Findings from Study 3 need to be interpreted cautiously. First, there were too few solvers in each group of conceptually- and procedurally-stronger solvers to draw firm conclusions. Typically, one would expect a sample size of thirty solvers in the procedurally- and conceptually-stronger group to provide suitable estimates of average scores (Howell, 2002, p. 323). However, in this study, only 14 procedurallystronger and 11 conceptually-stronger solvers were available to draw inferences about eye-trajectories. Comparisons of eye trajectories reveal that solvers made more horizontal than vertical or diagonal trajectories, consistent with the focus on extracting information to prepare for multiplying numerators and denominators. Thus, the general approach to using eye tracking to explore fraction arithmetic seems reasonable. 
Second, reducers were more likely to be conceptually-stronger than procedurally-stronger solvers. However, some conceptually-stronger and some procedurally strong solvers reduced at least one of the presented problems. Furthermore, reducers also provided correctly reduced solutions for at least half of the problems in the study, suggesting that when solvers reduced, they tended to do so consistently. However, relatively few participants (i.e., about $25 \%$ ) used reduction in the study. Thus, there was insufficient data to draw any firm conclusion to support these my inferences about the role of reduction in problem solution. A future challenge is to identify and collect data from a larger sample of reducers.

Third, all of the reducible and cross-reducible problems presented to participants involved multiplication of denominators, where one of the denominators was a common multiple of 5 . In comparison, only $60 \%$ of the non-reducible problems involved multiplication of denominators where one of the denominators had a common multiplication of 5. Retrieval of multiplication facts where one of the components was a multiple of 5 is faster than retrieval of facts where neither of the components was a multiple of 5 both in children and in adults (Campbell \& Graham, 1988). Longer problem-solving times for non-reducible than reducible problems might also reflect solvers difficulty in multiplying denominators where neither denominator had a multiple of 5. Indeed when problems where neither denominator had a multiple of 5, there were no pair-wise differences among unit-reducible, crossreducible and non-reducible problems.

\section{Summary}

Overall, findings in this chapter provide evidence that that in the context of fraction multiplication, conceptually-stronger solvers were more likely to reduce fractions than procedurally-stronger solvers. I further classified solvers who reduced 
fractions as reducers. Reducers' attention on fraction components, compared to that of non-reducers, was consistent with the prediction that cross-reduction would be reflected in eye gazes across the numerators and denominators of fraction pairs.

Reducible problems were solved more quickly and efficiently than nonreducible problems. However, the latency benefit for reducible problems was not accounted for by selection of the reduction algorithm. Rather reducible solutions were solved more quickly because participants applied a two-step, rather than a three-step solution algorithm. Findings in this chapter also highlight comparisons of fixation dyads as a useful tool to investigate strategy selection and execution in the context of fraction arithmetic. 


\section{CHAPTER 7: GENERAL DISCUSSION AND IMPLICATIONS}

The goal of this dissertation was to assess if adults' understanding of fraction concepts and procedures influences whether they simplify problems by reducing fraction when they perform fraction arithmetic. In this section, I summarize the main findings of this dissertation and discuss their implications for understanding adults' strategy choices in fraction arithmetic, more generally. I will describe how the reduction procedure could be integrated into existing computational models of fraction arithmetic. Finally, I discuss the limitations of my studies and summarize my conclusions.

\section{Fraction Knowledge Profiles}

The first goal was to identify fraction knowledge profiles among adults. Cluster analysis of data from the Fraction Knowledge Assessment in Study 1 revealed that adults can be grouped into two profiles. Procedurally-stronger solvers performed better than expected on procedures given their knowledge of fraction concepts. This group answered questions that required procedural and conceptual knowledge with equal ability. Conceptually-stronger solvers performed better than expected on concepts given their understanding of fraction procedures. This group scored higher on the conceptual than the procedural items. These two profiles were also observed in Hallett et al. (2012) for middle-school children. My findings show that individual differences in fraction knowledge exists even for adults. People in these profiles did not differ in basic arithmetic skills, however, suggesting that the groupings did not simply reflect overall differences in mathematical abilities, but more specific knowledge about fractions.

The general distinction between conceptual and procedural knowledge and how people use these two different types of knowledge to solve problems has been of 
interest to researchers for the past few decades (Hallett et al., 2012; Rittle-Johnson \& Siegler, 1998; Rittle-Johnson et al., 2001; Siegler et al., 2013). Conceptual knowledge is knowledge that is rich in relationships and associations (Hiebert \& Lefevre, 1986), whereas procedural knowledge is the sequence of steps initiated in order to achieve a goal (Rittle-Johnson \& Siegler, 1998). A solver with a strong understanding of concepts would therefore have a network of facts and concepts that are strongly associated with one another, whereas a solver with a strong understanding of procedures would be able to easily retrieve a sequence of algorithmic steps, where selection and application of one step is systematically followed by selection and application of another step.

In the context of fraction knowledge, therefore, conceptually-stronger solvers should have a strong grasp of knowledge of whether a fraction such as $1 / 2$ might have a larger, smaller or equivalent value to other rational numbers such as 0.5 or 1.4 . Thus, conceptual knowledge highlights how two numbers are related to each other in terms of their magnitude values. Procedurally-stronger solvers, on the other hand, would have a strong grasp of knowledge of how to apply a sequence of steps in order to achieve a solution for any given fraction problem (i.e., fraction arithmetic problems such as $1 / 2+1 / 3$ or $1 / 4 \times 1 / 5)$. It is important to note that a solver could have both a strong understanding of concepts and procedures. However, procedurally-stronger solvers could find it easier to retrieve sequences of steps than to retrieve concepts. Likewise, conceptually-stronger solvers could find it easier to use available facts to retrieve associated concepts than to retrieve sequences of steps.

\section{Differences in Strategy Selection across Solver Profiles}

In this dissertation, I found that solvers whose relative strengths were in either conceptual or procedural fraction understanding differed in terms of the strategies 
they used to solve problems. Studies of strategy selection for whole number arithmetic shows that children and adults have many strategies at their disposal but vary their strategies according to problem characteristics and the context in which problems are presented (Huber et al., 2014; Imbo, Duverne \& LeMaire, 2007; Imbo, Vandieredonck \& Rosseel, 2007).

When children solved fraction arithmetic problems, they selected strategies based on certain characteristics of the problem such as the required operation and denominator-denominator relations (Siegler et al., 2011; Siegler \& Pyke, 2013). The same pattern was also observed in Study 1. I found that a sample of adults also selected strategies based on problem characteristics and that they used some of the same incorrect strategies as children. Furthermore, I also found that solvers' strategies differed based on individual differences in how solvers organize their fraction knowledge. In Study 1, the conceptually-stronger group produced more systematic strategy errors than the procedurally-stronger group.

Two of the systematic strategy errors - the independent whole number error and the wrong inversion error - were the same strategy errors made by middle-school students (Siegler et al., 2011; Siegler \& Pyke, 2013). In addition to these errors, some adults also made wrong fraction inversion errors, erroneously inverting the first rather than the second fraction. Solvers who made this error had most of the components of the strategy stored in memory and so presumably had forgotten which fraction to invert. This error may reflect a lack of conceptual understanding of why it is the second fraction that is inverted in the division procedure. Thus, solvers were able to perform the sequence of steps required to perform the division algorithm but were unable to retrieve the fact about which fraction to invert.

Some solvers were influenced by a combination of the required operation and 
denominator-denominator relation to systematically select the wrong strategies resulting in incorrect answers to the fraction problems. Study 1 showed that some solvers were influenced by a combination of the required operation and denominatordenominator relation to systematically select the wrong strategies resulting in incorrect answers to the fraction problems. For example, when solvers used the independent whole number strategy, solvers treated each fraction component as separate and applied the required operation on both numerators and denominators. Furthermore, this strategy was more frequently observed for different- than samedenominator addition and subtraction problems. Solvers were sensitive to and treated the different denominators as distinct components, whereas they were less likely to treat same denominators as distinct components when applying their selected algorithm. This strategy was correct for multiplication but was a strategy error for addition and subtraction. Thus, in these cases participants were more likely to select the wrong strategy for that operation, cued by the relations between the denominators.

Conversely, when they used the wrong fraction operation strategy on multiplication or division problems, solvers maintained the value of the denominator while applying the required operation on the numerator. This strategy was more frequently observed for same- than different-denominator multiplication. Solvers were sensitive to the common denominator of fractions within the problem, as well as the procedure of maintaining the value of the common denominator, and hence multiplied the numerators while maintaining the value of the denominator. This strategy would have been correct for addition and subtraction but was a strategy error for multiplication. Once again, this strategy error was a failure to retrieve the correct strategy for the operation required.

Procedurally-stronger solvers were more likely to be cued by the operation 
alone and were less susceptible to denominator relation cues. For example, when adding and subtracting fractions, procedurally-stronger solvers applied the steps of obtaining and maintaining a common denominator and applying the required operation on the numerator. When multiplying fractions, procedurally-stronger solvers multiplied numerators and denominators. Procedurally-stronger solvers systematically applied these steps regardless of the value of fractions components. These findings provide further insight into how people in both solver profiles organize their fraction knowledge in the context of fraction arithmetic.

Conceptually-stronger solvers appeared to be sensitive to the relationship between fraction components and use the relation between components (i.e., fraction denominators), as well as the required operation as cues to select strategies. In contrast, procedurally-stronger solvers organized their knowledge in terms of sequence of steps and were more likely to apply a sequence of algorithmic steps based on the required operation, which, if applied correctly, will always lead to the correct solution.

\section{Differences in Use of Reduction Across Studies}

Table 7.1 shows a comparison of the pattern reduction use across the critical factors (i.e., operation, denominator relationship, and solver knowledge) for each study and each condition. In the online study and on worked protocols, solvers used reduction more frequently for division than multiplication. In the online study, solvers were more likely to reduce same- than different-denominator problems for both multiplication and division whereas, on worked protocols, solvers were more likely to reduce solutions for different- than same-denominator division problems. In the online study, procedurally-stronger solvers were more likely to use reduction than conceptually-stronger solvers whereas the reverse was true in both parts of Study 2. 
To summarize, reduction was used more frequently for division than multiplication across studies. However, reduction use across denominator relationship and solver group varied across studies.

One possible explanation for the variability of reduction use is that it is influenced by task demands. In Study 1, solver completed problems at their own pace, without much explicit instruction. In contrast, for the worked solutions in Study 2 (Part I), solvers had to show exactly how they solved each problem in writing and explain their solution process. For the computer-based trials in Study 2 (Part II), participants were instructed to solve problems as quickly and accurately as possible, without access to paper and pencil. These varying task demands may have influenced whether reduction was useful in producing a correct solution.

Another possible explanation for the variations in reduction use was that the problem sets also varied across studies. For the FARRA problems in Study 1, all numerators and denominators were single-digit numbers with magnitude of 5 or less. In Study 2, the problems for the worked protocols were all reducible problems with at least one multi-digit denominator (with a multiple of 5), and as such were more difficult that the FARRA problems. The computer-based problems were all multiplication problems, many had two double-digit denominators, and not all problems were reducible. Variations in the problems may have influenced whether reduction was viewed as useful or relevant.

A third possible explanation for why reduction use varied across studies is that the groups of participants appeared to have different levels of overall fraction knowledge. Participants who volunteered for Study 2 were a subset of the participants who completed the online study -- a comparison of relative and overall procedural fraction knowledge scores suggests that these conceptually-stronger solvers were 
comparable in procedural knowledge to conceptually-stronger solvers (See Table 5.2). In contrast, for the online study, the procedurally-stronger solvers were better in procedural knowledge than conceptually-stronger solvers (See Figures 3.2 and 3.3). Different levels of procedural knowledge across solver profiles in each study could account for conceptually-stronger solvers preference to reduce in Study 2 when multiplying fractions, and procedurally-stronger solvers preference to reduce fractions when dividing fractions in Study 1.

In summary, frequency of reduction appears to be cued by the required operations. Specifically, reduction is more frequently selected for division problems. However, denominator-relationship and solver knowledge also appear to be related to reduction use, but also vary with other problem characteristics and instructions provided to solvers. These other factors will be explored further in the limitations section at the end of the chapter. 
Table 7.1. Comparison of effects of factors on correct reduction-use across studies for multiplication and division problems presented across studies.

\begin{tabular}{lccc}
\hline & Operation & Denominator-Relationship & Solver knowledge \\
\hline & Division $>$ multiplication & Study 1: Online Study (FARRA problems) & Procedurally-stronger $>$ conceptually stronger \\
& & Same $>$ different & (division) \\
\hline & & Study 2: Part 1: worked protocols & No effect \\
Overall & Division $>$ multiplication & No effect & No effect \\
Reduction- & Division $>$ multiplication & No effect & Conceptually-stronger $>$ procedurally stronger \\
Reduction- & No effect & Different $>$ same & (division) \\
after-solution & & & Conceptually-stronger $>$ procedurally stronger \\
\hline
\end{tabular}


The finding that reduction is less frequently observed for multiplication than division suggests that solvers were more likely to directly multiply numerators and denominators than to apply reduction strategies when multiplying fractions. In contrast, solvers were more likely to reduce numerator-denominator pairs rather than directly apply the division algorithm when dividing fractions. Reducing fractions within a problem is not considered a rote procedure because the reduction algorithm cannot be applied to all division problems; instead, use of reduction suggests that solvers are using problem characteristics to modify their solution strategies. Denominator relations also interacted with operation to cue the reduction algorithm. Solvers applied the reduction algorithm more frequently for different denominator than same denominator division. However, when solvers multiplied fractions, reduction was applied with equal frequency for same- and different-denominator problems. These findings highlight that denominator relationship interact with operation to facilitate selection of the reduction algorithm.

Comparisons of conceptually- and procedurally-stronger solvers among reducers further suggests that conceptually-stronger solvers were more likely than procedurally-stronger solvers to reduce fractions, suggesting that fraction knowledge was also a factor in determining whether a solver decided to reduce fractions. Individual differences in reduction use can be accounted for by individual differences in how people in each solver profile organize their fraction knowledge. I suggest that conceptually-stronger solvers, because of their strengths in finding associations, were more sensitive to the presence of common factor numerator-denominator pairs and used the common factor relationship as a cue to select and apply the reduction algorithm. Procedurally-stronger solvers, because of their strengths in retrieving and sequentially applying algorithmic steps, were more likely to directly apply the 
multiplication algorithm when multiplying fractions, instead of applying the reduction algorithm, even for complicated problems.

Another possible explanation for why procedurally-stronger solvers did not reduce problems was because they were not able to apply reduction. However, Study 2 also provides evidence that procedurally-stronger solvers were more likely to apply the reduction algorithm than conceptually-stronger solvers after obtaining the solution. Thus, procedurally-stronger solvers appeared to understand the conditions for reduction (i.e., the presence of common factors) and how to apply the reduction algorithm on the final solution. They either did not choose to simplify the fractions before applying the procedure or possibly did not have a reduction-before-solution procedure in their repertoire.

These results highlight a possible link between individual differences in the organization of fraction knowledge and reduction use. Conceptually-stronger solvers, because of their strengths in finding associations between components, were more likely to, identify relations between fraction components, which in turn influenced the strategies that they selected. Procedurally-stronger solvers, because of their strengths in retrieving and applying sequence of steps, preferred to approach fraction arithmetic problems by using the relevant operation as a cue to retrieve and apply the required procedure for the operation, regardless of the components of each fraction in the problem.

Evidence of representational differences across solver profiles was also provided by Geller, Son and Stigler (2017). Geller et al. asked participants to explain their reasoning while comparing fractions and found a relationship between participants' performance and the conceptual sophistication that participants used to explain their answers. Participants who explained answers in terms of rules were most 
accurate and compared fractions more quickly than solvers who reasoned out their answers according to conceptual explanations.

According to Geller et al. (2017), solvers who used a procedural approach have knowledge of applying a rule to obtain a correct solution, but also implicitly have a conceptual knowledge of the rule because they understand that the rule, if correctly applied, will produce the correct solution. Hence there is no need to invoke a conceptual explanation even though a solver might have knowledge of concepts. In contrast, solvers who adopted a conceptual approach had difficulty using procedures to perform fraction comparisons, and therefore had to resort to the slower conceptual approach to compare fractions. The difficulty that conceptual solvers had with using procedures suggests that these solvers were not fully able to integrate conceptual and procedural information.

In the context of fraction arithmetic, Geller et al.'s observations dovetail with my findings that individual differences exist across solver profiles that are related to solvers' use of procedural rules (i.e., a procedural approach) versus reasoning out their answers by forming a conceptual representation of the problem and identifying associations between components of the problem (i.e., a conceptual approach). Procedurally-stronger solvers directly applied rules to solve problems, whereas conceptually-stronger solvers used their understanding of concepts to identify associations among fraction components and used these associations as cues to select problem-solving strategies. Thus, procedurally-stronger solvers tended to select and apply rules to solve fraction arithmetic problems, whereas conceptually-stronger solvers tended to select problem-solving strategies based on associations between fraction components, and thus varied their strategies based on problem characteristics. 


\section{A Framework of Reduction Use in Fraction Multiplication}

In summary, the results of this thesis provided evidence that reduction was applied differentially according to solver and problem characteristics. In particular, conceptually-stronger solvers were more likely to reduce fractions than procedurallystronger solvers on multiplication problems. When I further classified solvers who reduced fractions as reducers, I found that their patterns of eye trajectories on fraction components were consistent with the predicted trajectories for the procedure for cross-reduction. Moreover, reducible problems were solved more quickly and accurately than non-reducible problems. However, the latency benefit for reducible problems was not accounted for by selection of the reduction algorithm. Instead, reducible solutions were solved more quickly because participants applied the twostep algorithm rather than the three-step algorithm. Performance on non-reducible problems was also influenced by computational complexity of those problems: Solvers were slower and less accurate multiplying denominators that were not multiples of 5, a feature that only occurred on non-reducible problems. Accordingly, when only problems with denominators that were multiples of 5 were analyzed, the latency benefit for the two types of reducible problems diminished, indicating that numerical complexity influenced problem solving latencies.

Recall that the two-step algorithm is the default strategy for multiplication, which, if applied correctly, will always produce the correct solution. One step requires solvers to multiply numerators, while another step requires solvers to multiply denominators. This two-step procedure for multiplication is taught to middle-school students. They learn to associate the multiplication operation with the procedure and are eventually cued by the required operation (i.e., multiplication) to apply the algorithm (Braithwaite et al., 2017; Braithwaite, Leib, Siegler, \& McMullen, 2019). 
However, when a reducible problem with a common factor in the numeratordenominator pair is presented to participants, conceptually- and procedurally-stronger solvers appeared to use different cues to select and apply the reduction algorithm. The possibility of reduction, therefore, leads to two possible elaborations of the algorithm for reducible multiplication problems (See Table 7.2).

Table 7.2. Two possible elaborations of the algorithm for reducible multiplication problems: The reduction after multiplication versus reduction before multiplication.

\begin{tabular}{lll}
\hline Step & Reduction after multiplication & Reduction before multiplication \\
\hline 1 & Multiply numerators (or & Check problem for common factor in \\
& denominators) & numerator-denominator pair of solution \\
2 & Multiply denominators (or & If possible, reduce numerator- \\
& numerators) & denominator pair of problem \\
3 & Check solution for common factor & Multiply numerators (or denominators) \\
& in numerator-denominator pair of & \\
& solution & \\
& If possible, reduce numerator- & Multiply denominators (or numerators) \\
& denominator pair of solution & \\
\hline
\end{tabular}

Reduction after multiplication. One group of solvers are cued by the operation and directly apply the two-step multiplication algorithm. However, after applying the algorithm to obtain a solution, some solvers further applied the reduction algorithm to the solution. Step 3 in this modified algorithm is to check the solution for common factors. Step 4 is to reduce the answer by dividing the numerator and denominator by the common factor. This sequence is consistent, more generally, with a procedural approach to fraction arithmetic. Solvers are directly cued by the 
multiplication operation, apply the multiplication algorithm to obtain a solution, then subsequently apply the reduction algorithm on the solution to obtain a simplified solution. It is possible that for solvers who use this algorithm, the link between the required operation and the procedure has become so well-learned that they have difficulty inhibiting the direct multiplication algorithm to apply the reduction algorithm.

Reduction before multiplication. Another group of solvers identified the common numerator-denominator pair within the problem and applied the reduction algorithm before applying the multiplication algorithm. This sequence of steps is consistent, more generally with a conceptual approach to solving fraction problems. Solvers identified and used associations between fraction components as cues to select and apply strategies. When multiplying fractions, solvers identified the reducible numerator-denominator pair, applied the reduction procedure, then subsequently applied the multiplication algorithm.

\section{Reduction Involves Both Conceptual and Procedural Knowledge}

The question of which solver profile is more likely to reduce fractions is of theoretical interest in mathematical cognition because reducing fractions could hypothetically tap into both conceptual and procedural knowledge. Reduction taps into procedural knowledge, because in order to perform reduction, solvers need to be able to perform a sequence of steps. Application of the reduction procedure requires solvers identify common factors in a numerator-denominator pair, then divide both the numerator and denominator pair by the common factor. Thus, reduction also taps into conceptual knowledge because reducing a numerator-denominator pair within the problem does not change the value of the solution when performing the required operation. 
The underlying conceptual knowledge required for reduction is knowledge about fraction equivalence, for example, $2 / 4$ is equivalent to $1 / 2$. Someone could have conceptual understanding of fraction equivalence but nevertheless apply the procedure incorrectly. It is also possible that a person could reduce fractions without understanding why this is an acceptable step, if they do not have an understanding of fraction equivalence but have just learned the reduction procedure. In summary, the reduction procedure appears to tap into both conceptual and procedural fraction knowledge.

Solvers need to be able to retrieve and apply the appropriate sequence of steps for fraction multiplication (i.e., use procedural knowledge), regardless of their relative strengths in concepts and procedures or whether they apply a reduction procedure. Solvers could apply the multiplication algorithm to all problems and obtain the correct solution without any knowledge of concepts. That is how the FARRA program operates. How then does conceptual knowledge play a role in fraction arithmetic? I provided evidence that solvers who reduced fractions (i.e., reducers) were more likely to be conceptually-stronger that procedurally-stronger solvers. Using reduction seems to implicate conceptual knowledge because solvers need to understand that the numerator and denominator are related by a common factor, and they need to identify the common factor to apply the reduction procedure. Another possibility is that procedurally-stronger solvers apply arithmetic rules to solve fraction problems, whereas conceptually-stronger solvers reason out their answers by drawing upon their knowledge of associations between components (i.e., that reducible numerator-denominator pairs share a common factor) and fraction principles (i.e., a fraction that is reduced also has the same value as the original fraction). This latter approach would be consistent with the interpretation of Geller et al. (2017) that 
solvers use rules to solve problems but also use concepts to reason out answers for problems that they find difficult.

Why did conceptually-stronger solvers applied the reduction procedure more frequently than procedurally-stronger solvers? There are two possibilities. One possibility is that procedurally-stronger solvers were able to multiply large numbers better than conceptually-stronger solvers and hence there was no need for procedurally-stronger solvers to reduce fractions. The second possibility is that conceptually-stronger solvers had a better understanding than procedurally-stronger solvers of suitable and appropriate contexts when applying the reduction algorithm is useful to solve a problem. Evidence from written protocols suggests that procedurallystronger solvers actually made more computational errors than conceptually-stronger solvers when multiplying large components. Furthermore, performance on the singleand multi-digit multiplication tasks (i.e., BMT and CFT) suggests that both groups of solvers were equally proficient in their ability to perform multiplication. Thus, the first possibility, that procedurally-stronger solvers are better at multiplication than conceptually-stronger solvers, is not supported by the data. Instead, I suggest that conceptually-stronger solvers have an understanding of how to reduce a fraction and of the circumstances in which reduction is useful. Thus, applying the reduction procedure in the correct context entails both conceptual and procedural fraction knowledge.

\section{Implications for the FARRA model}

The FARRA model provides a detailed account of the selection and application of fraction procedures used to solve fraction arithmetic for each of the four operations but it lacks conceptual knowledge (Braithwaite et al., 2017; Braithwaite et al., 2019). FARRA applies sequences of steps to solve fraction 
arithmetic problems. It selects the steps by matching the features of the problem to stored procedures. Because FARRA lacks a knowledge base that includes information about relations among fractions components, it is unable to use a procedure that involves identifying an association between components - hence, reduction before multiplication or division is outside the scope of the model. Accordingly, the reduction procedure has not been implemented in FARRA.

However, this dissertation has provided evidence that the reduction algorithm is in the repertoire of strategies that solvers use to perform fraction arithmetic and that solvers' knowledge of fractions also influences selection of the reduction algorithm. How should reduction be implemented in a computational model like FARRA? Selecting the reduction algorithm also involves knowledge of factorization. Reduction could therefore be implemented in FARRA by including production rules which specify the strategy and execution rules for factorization, although the revised model still needs to be tested. The typical steps in the context of reducing a fraction involve first identifying whether a numerator-denominator pair of a fraction share a common factor. The numerator and the denominator are then divided by the common factor to obtain a reduced fraction. I have proposed how these steps might be implemented in FARRA for multiplication (See Table 7.3). 
Table 7.3. Strategy rules for implementing reduction in FARRA

\begin{tabular}{|c|c|c|c|}
\hline Rule & $\begin{array}{l}\text { Strategy } \\
\text { Name }\end{array}$ & Condition & Action \\
\hline $1 \mathrm{a}$ & $\begin{array}{l}\text { Factor } \\
\text { numerator }\end{array}$ & numerator $>1$ & $\begin{array}{l}\text { Identify all factors of numerator and } \\
\text { hold factors of numerator in } \\
\text { memory }\end{array}$ \\
\hline $2 \mathrm{a}$ & $\begin{array}{l}\text { Factor } \\
\text { denominator }\end{array}$ & denominator $>1$ & $\begin{array}{l}\text { Identify all factors of denominator } \\
\text { and hold factors of denominator in } \\
\text { memory }\end{array}$ \\
\hline $3 a$ & $\begin{array}{l}\text { Check } \\
\text { common } \\
\text { factor }\end{array}$ & Operation is $\times$ & $\begin{array}{l}\text { Check factor of each numerator and } \\
\text { factor of each denominator }\end{array}$ \\
\hline $4 a$ & Factorize & $\begin{array}{l}\text { One factor of } \\
\text { numerator is equal } \\
\text { to one factor of } \\
\text { denominator }\end{array}$ & $\begin{array}{l}\text { Delete factor of numerator and } \\
\text { factor of denominator. Set } \\
\text { numerator to result of } \\
\text { multiplication of remaining factors } \\
\text { of numerator. Set denominator to } \\
\text { result of multiplication of } \\
\text { remaining factors of denominator }\end{array}$ \\
\hline
\end{tabular}

These steps highlight the procedural approach to implementing reduction and thus can be implemented in a model like FARRA that lacks conceptual knowledge. However, this approach also raises the further question of how conceptual fraction knowledge might influence selection of the reduction strategy. A possible answer is that reduction does not require conceptual fraction knowledge of equivalence at all, rather it requires the conceptual understanding of factorization and why reduction is useful in the context of solving fraction arithmetic (i.e., it simplifies the problem). The understanding of why reduction is useful in the context of fraction arithmetic could be considered conceptual knowledge, because it requires that solvers understand why they are using the algorithm. However, solvers could quite conceivably apply reduction without tapping into the understanding of the efficacy of reduction in solving complicated problems. Using reduction is consistent with a procedurallystronger approach in which solvers directly apply procedures without drawing upon the knowledge of the efficacy of reduction in the fraction arithmetic. 
In summary, conceptual fraction knowledge may not be critical to selecting and applying the reduction algorithm. Instead, knowledge of how fraction components within each problem are related to one another and how principles of other arithmetic knowledge (i.e., factorization) may be effectively used as an aid to simplify problems.

\section{Limitations}

The findings that describe reduction in this dissertation are exploratory, and hence there are several confounds that might provide alternative interpretations to the findings. These confounds can be categorized as participant confounds, problem confounds and instruction confounds. I describe these confounds in dissertation in this section, along with the limitation of drawing interpretations from multiple analyses and suggest ways to address them in the next section "Future Directions".

\section{Instruction Confounds}

The instructions provided to participants varied across studies and for both parts of Study 2. For Study 1, participants were told to solve fraction problems, but were given free rein to solve fraction problems under conditions of their own choice. In contrast, in the written protocol part of Study 2, participants were told to solve problems and provide worked protocols. For the computer-based part of Study 2, participants were told to solve problems as quickly and accurately as possible. Furthermore, solvers were not instructed to reduce fractions, thus some of the observations, especially for the protocol studies could be confounded by the fact that solvers who could have reduced fractions, did not do so, because they were not instructed to reduce. Thus, the conclusions are limited to these situations where reduction was not mentioned.

The instructions provided to participants in the computer-based study might have also favoured selection of the direct multiplication algorithm. Participants might 
have interpreted instructions that they were required to solve problems accurately using the more efficient strategy possible. Hence participants selected the shorter and presumably more efficient two-step direct multiplication procedure, rather than the three-step procedure that included reduction.

\section{Problem Confounds}

Problems chosen for the studies might also have other characteristics that contributed to the observed results. In Part I of Studies 2 and 3, problems were constructed based on whether they shared a common denominator. The denominators of each problem were then maintained across operations. However, as a result of this manipulation, fractions in different-denominator problems consisted of one singledigit denominator and one double-digit denominator, whereas fractions in samedenominator problems consisted of two fractions with double-digit denominators. As a result of this manipulation, the observation that people made more errors when they multiplied different-denominator than same-denominator problems could also be accounted for by their difficulties with multiplying double-digit denominators.

\section{Participant Confounds}

Participants' knowledge of fractions also varied between participants who participated in the online study and the subset of participants who also volunteered to participate in Study 2. A comparison of relative and overall procedural fraction knowledge scores for conceptually and procedurally-stronger solvers suggests that in the online study, procedurally-stronger solvers performed better on the procedural FKA measure than conceptually-stronger solvers. However, there was no difference on overall procedural FKA scores for conceptually and procedurally-strong solvers who volunteered to participate in Study 2.

Although all respondents on the online study were invited to participate in 
Study 2, respondents were also told that they were to solve fraction arithmetic problems. It is therefore possible that volunteers in Study 2 consisted mostly of respondents who felt comfortable working with fraction arithmetic. This potential bias was reflected in Study 2 participants' overall conceptual and procedural scores. There was no difference in procedural scores for conceptually- and procedurally-stronger solvers in Study 2, despite a significant difference for the larger groups in Study 1.

Another participant-based limitation involves the identification of fraction knowledge profiles used to study reduction. The original goal was to isolate whether reduction was either conceptual or procedural knowledge. With this goal in mind, I performed cluster analysis on residualized scores such that profiles were identified based on fraction concepts and fraction procedures and found evidence that adults could be profiled as conceptually-stronger and procedurally-stronger solvers.

However, both conceptually and procedurally stronger solvers reduced fractions. Furthermore, participants who were classified as reducers only reduced a subset of presented problems but did not reduce for all problems. Inferences drawn from accuracy, response time, and fixation dyad analyses thus included comparisons that included both reduced and non-reduced problems, weakening the inference that differences for these measures was related to solvers' use of reduction. Thus a better way to study the types of fraction knowledge profiles that select the reduction procedure might have been to identify and recruit a larger sample of solvers who reduced problems accurately and further compare these solvers' fraction knowledge and basic arithmetic scores with solvers who were not able to reduce fractions.

\section{Multiple Analyses}

A fourth limitation is that many analyses were done in this dissertation, increasing the possibility of Type I errors. The probability of making Type I errors 
was controlled for each separate analysis by using standard multiple comparison corrections which adjust the p-value based on the number of comparisons being made. However, multiple analyses were performed in the dissertation, and overall experiment-wise error rates were not controlled. Thus, the probability of a Type 1 error being present in the dissertation is larger than .05 (the standard convention in psychological studies). Mitigating this problem, the dissertation is exploratory, because no previous research has been performed on reduction. Nevertheless, findings in the dissertation should be treated with caution. Follow-up studies are needed to determine whether inferences drawn from these findings can be replicated.

\section{Future Directions}

One avenue of future research requires extension of findings from this study to a more representative sample of reducers. The studies in the dissertation examined the relationship between solvers' knowledge of fraction concepts and procedures and their use of the reduction procedure. However, even though I found that conceptuallystronger solvers were more likely to reduce problems than procedurally-stronger solvers, both conceptually- and procedurally-stronger solvers reduced fractions. Thus, it was not the case that only solvers who had relatively better knowledge of concepts preferred to reduce fractions. One approach is to obtain a sample where reduction was used more frequently and then compare fraction knowledge and basic arithmetic scores for solvers who did or did not use reduction. Identifying each groups' strengths and weaknesses on fraction and arithmetic scores might be a better way to identify the factors that determine whether a solver can reduce effectively and accurately.

Another way to address the limitation of the small sample of reducers, and also address instruction confounds, is to explicitly request solvers to perform reduction, rather than letting the participant decide whether to reduce. Providing 
instructions to reduce solutions such as "The problems you will see can be converted into a simpler problem by using reduction. Please try to use reduction when solving these problems and be as accurate as possible." would ensure that solvers will be specifically informed to use reduction, and hence provide a larger and more representative sample of reducers.

A third avenue of research would to be to assess reduction use with a different set of problems and/or extend comparisons of latencies and ocular measures of reduction use into division problems. Comparisons of reduction use suggests that solvers preferred to reduce fractions when dividing but were less likely to use reduction when multiplying. However, although accuracy rates for division were assessed in three studies, latencies and eye trajectories for division were not assessed. Thus, comparisons of latencies and ocular measures when dividing fractions is also a promising avenue for studying reduction, because division problems were reduced more frequently than multiplication.

A fourth avenue of investigation would be to implement the reduction procedure in a computational model like FARRA. Currently, reduction has not been implemented in FARRA or any other computational model. I have suggested the steps required to implement reduction. The model then needs to be tested with a suitable sample of solvers to assess the accuracy of the model.

\section{Conclusions}

In conclusion, my dissertation is novel because it identifies possible factors that influence solvers' selection of the reduction procedure. I found that the required operation and the denominator-denominator relation for fraction pairs influenced use of reduction. Furthermore, solvers' knowledge of fraction concepts and procedures was related to selection of the reduction procedure. I provide a framework of how 
reduction is applied differently among two groups of solvers when multiplying fractions. I also suggest how the reduction procedure could be integrated into a model of fraction arithmetic. Finally, I suggest that applying the reduction procedure in the appropriate context requires an understanding of fraction procedures, but also taps into solvers' understanding of factorization and the conceptual knowledge of why reduction is useful in the context of fraction arithmetic. 


\section{CHAPTER 8: REFERENCES}

Bailey, D. H., Hansen, N., \& Jordan, N. C. (2017). The codevelopment of children's fraction arithmetic skill and fraction magnitude understanding. Journal of Educational Psychology, 109,509-518.

Behr, M. J., Wachsmuth, I., \& Post, T. R. (1985). Construct a sum: A measure of children's understanding of fraction size. Journal for Research in Mathematics Education, 15, 120-131.

Bentley, B., \& Bossé, M. J. (2018). College students' understanding of fraction operations. International Electronic Journal of Mathematics Education, 13, 233-247.

Bezuk, N., \& Cramer, K. (1989). Teaching about fractions: What, when, and how? In P. Trafton (Ed.), National council of teachers of mathematics 1989 year book: New directions for elementary school mathematics (pp156-167). Reston, VA: National Council of Teachers of Mathematics.

Booth, J. L., \& Newton, K. J. (2012). Fractions: Could they really be the gatekeeper's doorman?. Contemporary Educational Psychology, 37, 247-253.

Braithwaite, D. W., Leib, E. R., Siegler, R. S., \& McMullen, J. (2019). Individual differences in fraction arithmetic learning. Cognitive psychology, 112, 81-98.

Braithwhite, D.W., Pyke, A. A., \& Siegler, R.S. (2017). A Computational Model of Fraction Arithmetic. Psychological Review, 124, 603-625.

Braithwaite, D. W., \& Siegler, R. S. (2018). Children learn spurious associations in their math textbooks: Examples from fraction arithmetic. Journal of Experimental Psychology: Learning, Memory, and Cognition, 1765-1777.

Brown, J. S., \& VanLehn, K. (1980). Repair theory: A generative theory of bugs in procedural skills. Cognitive Science, 4, 379-426. 
Brown, M., Hart, K., \& Küchemann, D. (1984). Chelsea Diagnostic Mathematics Tests. Fractions 1. Incorporating Fractions 1 (Computation). Windsor, England. NPER-Nelson.

Byrnes, J. P. (1992). The conceptual basis of procedural learning. Cognitive Development, 7, 235-237.

Byrnes, J. P., \& Wasik, B. A. (1991). Role of conceptual knowledge in mathematical procedural learning. Developmental Psychology, 27, 777-786.

Carpenter, T., Corbitt, M., Kepner, H., Lindquist, M., \& Reys, R. (1980). Results of the second NAEP mathematics assessment: Secondary school. Mathematics Teacher, 73, 329-338.

Caliński, T., \& Harabasz, J. (1974). A dendrite method for cluster analysis. Communications in Statistics-Theory and Methods A, 3, 1-27.

Campbell, J. I., \& Graham, D. J. (1985). Mental multiplication skill: Structure, process, and acquisition. Canadian Journal of Psychology/Revue canadienne de psychologie, 39, 338-366.

Crooks, N. M., \& Alibali, M. W. (2014). Defining and measuring conceptual knowledge in mathematics. Developmental Review, 34, 344-377.

Curtis, E. T., Huebner, M. G., \& LeFevre, J. A. (2016). The relationship between problem size and fixation patterns during addition, subtraction, multiplication, and division. Journal of Numerical Cognition, 2, 91-115.

Duda, R. O., \& Hart, P. E. (1973). Pattern classification and scene analysis. New York: Wiley, 1973.

Freudenthal, H. (2002). Fractions. Didactical phenomenology of mathematical structures, 133-177. Mathematics Education Library, vol 1. Springer, Dordrecht 
Fuchs, L. S., Compton, D. L., Fuchs, D., Powell, S. R., Schumacher, R. F., Hamlett, C. L., . . Vukovic, R. K. (2012). Contributions of domain general cognitive resources and different forms of arithmetic development to pre-algebraic knowledge. Developmental Psychology, 48, 1315-1326.

Fuchs, L. S., Geary, D. C., Compton, D. L., Fuchs, D., Hamlett, C. L., Seethaler, P. M., . . Schatschneider, C. (2010). Do different types of school mathematics development depend on different constellations of numerical versus general cognitive abilities? Developmental Psychology, 46, 1731-1746.

Geary, D. C., Hoard, M. K., Byrd-Craven, J., Nugent, L., \& Numtee, C. (2007). Cognitive mechanisms underlying achievement deficits in children with mathematical learning disability. Child Development, 78, 1343-1359.

Geller, E. H., Son, J. Y., \& Stigler, J. W. (2017). Conceptual explanations and understanding fraction comparisons. Learning and Instruction, 52, 122-129.

Hallett, D., Nunes, T., \& Bryant, P. (2010). Individual differences in conceptual and procedural knowledge when learning fractions. Journal of Educational Psychology, 102, 395-406.

Hallett, D., Nunes, T., Bryant, P., \& Thorpe, C. M. (2012). Individual differences in conceptual and procedural fraction understanding: The role of abilities and school experience. Journal of Experimental Child Psychology, 113, 469-486.

Hansen, N., Jordan, N. C., Fernandez, E., Siegler, R. S., Fuchs, L., Gersten, R., \& Micklos, D. (2015). General and math-specific predictors of sixth-graders' knowledge of fractions. Cognitive Development, 35, 34-49.

Hecht, S. A. (1998). Toward an information-processing account of individual differences in fraction skills. Journal of Educational Psychology, 90, 545-559. Hecht, S. A., \& Vagi, K. J. (2012). Patterns of strengths and weaknesses in children's 
knowledge about fractions. Journal of Experimental Child Psychology, 111, 212-229.

Hiebert, J., \& Lefevre, P. (1986). Conceptual and procedural knowledge in mathematics: An introductory analysis. In J. Hiebert (Ed.), Conceptual and procedural knowledge: The case of mathematics (pp. 1-27). Hillsdale, NJ: Lawrence Erlbaum.

Howell, D. C. "Statistical methods for psychology 5th edition." Belmont, CA: Thomson Wadsworth (2002).

Huber, S., Moeller, K., \& Nuerk, H. C. (2014). Adaptive processing of fractionsEvidence from eye-tracking. Acta Psychologica, 148, 37-48.

Hurst, M., \& Cordes, S. (2016). Rational-number comparison across notation: Fractions, decimals, and whole numbers. Journal of Experimental Psychology: Human Perception and Performance, 42, 281-293.

Hurst, M. A., \& Cordes, S. (2018). Children's understanding of fraction and decimal symbols and the notation-specific relation to pre-algebra ability. Journal of Experimental Child Psychology, 168, 32-48.

Ischebeck, A., Weilharter, M., \& Körner, C. (2016). Eye movements reflect and shape strategies in fraction comparison. Quarterly Journal of Experimental Psychology, 69, 713-727.

Imbo, I., Duverne, S., \& Lemaire, P. (2007). Working memory, strategy execution, and strategy selection in mental arithmetic. The Quarterly Journal of Experimental Psychology, 60, 1246-1264.

Imbo, I., Vandierendonck, A., \& Rosseel, Y. (2007). The influence of problem features and individual differences on strategic performance in simple arithmetic. Memory \& Cognition, 35, 454-463. 
Jarmasz, J., \& Hollands, J. G. (2009). Confidence intervals in repeated-measures designs: The number of observations principle. Canadian Journal of Experimental Psychology/Revue canadienne de psychologie expérimentale, 63(2), 124.

Jordan, N. C., Hansen, N., Fuchs, L. S., Siegler, R. S., Gersten, R., \& Micklos, D.(2013). Developmental predictors of fraction concepts and procedures. Journal of Experimental Child Psychology, 116, 45-58

LeFevre, J. A., Kulak, A. G., \& Heymans, S. L. (1992). Factors influencing the selection of university majors varying in mathematical content. Canadian Journal of Behavioural Sciencel 24, 276-289.

Lortie-Forgues, H., Tian, J., \& Siegler, R. S. (2015). Why is learning fraction and decimal arithmetic so difficult? Developmental Review, 38, 201-221. Journal of Educational Psychology, 107, 909-918.

McCloskey, M. (1992). Cognitive mechanisms in numerical processing: Evidence from acquired dyscalculia. Cognition, 44, 107-157.

McCloskey, M., Caramazza, A., \& Basili, A. (1985). Cognitive mechanisms in number processing and calculation: Evidence from dyscalculia. Brain and Cognition, 4, 171-196.

Olive, J. (1999). From fractions to rational numbers of arithmetic: A reorganization hypothesis. Mathematical thinking and learning, 279-314.

Payne, S. J., \& Squibb, H. R. (1990). Algebra mal-rules and cognitive accounts of error. Cognitive Science, 14, 445-481.

Rickard, T. C. (2005). A revised identical elements model of arithmetic fact representation. Journal of Experimental Psychology: Learning, Memory, and Cognition, 31, 250-257. 
Rickard, T. C., \& Bourne Jr, L. E. (1996). Some tests of an identical elements model of basic arithmetic skills. Journal of Experimental Psychology: Learning, Memory, and Cognition, 22, 1281-1296.

Rickard, T. C., Healy, A. F., \& Bourne, L. E. (1994). On the cognitive structure of basic arithmetic skills: Operation, order, and symbol transfer effects. Journal of Experimental Psychology: Learning, Memory, and Cognition, 20, 11391153.

Rittle-Johnson, B. (2017). Developing Mathematics Knowledge. Child Development Perspectives.11, 184-190.

Rittle-Johnson, B., \& Schneider, M. (2015). Developing conceptual and procedural knowledge of mathematics. In R. C. Kadosh \& A. Dowker (Eds.), Oxford handbook of numerical cognition (pp. 1102-1118). Oxford: Oxford University Press.

Rittle-Johnson, B., Schneider, M., \& Star, J. R. (2015). Not a one-way street: Bidirectional relations between procedural and conceptual knowledge of mathematics. Educational Psychology Review, 27, 587-597.

Rittle-Johnson, B., \& Siegler, R. S. (1998). The relation between conceptual and procedural knowledge in learning mathematics: A review. In C. Donlan (Ed.), The development of mathematical skill (pp. 75-110). Hove, UK: Psychology Press.

Rittle-Johnson, B., Siegler, R. S., \& Alibali, M. W. (2001). Developing conceptual understanding and procedural skill in mathematics: An iterative process. Journal of Educational Psychology, 93, 346-362.

Rittle-Johnson, B., \& Star, J. (2007). Does comparing solution methods facilitate conceptual and procedural knowledge? An experimental study on learning to 
solve equations. Journal of Educational Psychology, 99, 561-574.

Siegler, R. S. (1988). Strategy choice procedures and the development of multiplication skill. Journal of Experimental Psychology: General, 117, 258-275.

Siegler, R. S. (1991). Strategy choice and strategy discovery. Learning and Instruction, 1, 89-102.

Siegler, R. S., Fazio, L. K., Bailey, D. H., \& Zhou, X. (2013). Fractions: the new frontier for theories of numerical development. Trends in Cognitive Sciences, 17, 13-19.

Siegler, R. S., \& Lortie-Forgues, H. (2015). Conceptual knowledge of fraction arithmetic. Journal of Educational Psychology, 107, 909-918.

Siegler, R. S., \& Pyke, A. A. (2013). Developmental and individual differences in understanding of fractions. Developmental Psychology, 49, 1994-2004.

Siegler, R. S., Thompson, C. A., \& Schneider, M. (2011). An integrated theory of whole number and fractions development. Cognitive Psychology, 62, 273-296.

Stigler, J. W., Givvin, K. B., \& Thompson, B. J. (2010). What community college developmental mathematics students understand about mathematics. MathAMATYC Educator, 1, 4-16.

Sowinski, C., Dunbar, K., \& LeFevre, J. (2014). Calculation Fluency Test, unpublished technical report, Math Lab, Carleton University, Ottawa, Canada

Tan, S. Muldner, K. \& LeFevre, J. A. (2016, August). Solution of division by access to multiplication: Evidence from eye tracking. 38th Annual Conference of the Cognitive Science Society, 10 to 13th August. Philadelphia, Pennsylvania. The Ontario Curriculum (2018). 
http://www.edu.gov.on.ca/eng/curriculum/elementary/math18curr.pdf). Accessed $15^{\text {th }}$ January, 2020.

Ward, J. H., Jr. (1963). Hierarchical Grouping to Optimize an Objective Function. Journal of the American Statistical Association, 58, 236-244.

Zbrodoff, N. J., \& Logan, G. D. (2005). What everyone finds: The problem-size effect. In J. I. D. Campbell (Ed.), Handbook of Mathematical Cognition (p. 331-346). New York: Psychology Press. 


\section{APPENDIX A: RULES IMPLEMENTED IN FARRA}

Strategy rules implemented in FARRA

\begin{tabular}{|c|c|c|c|}
\hline Rule & $\begin{array}{l}\text { Strategy } \\
\text { Name }\end{array}$ & Condition & Action \\
\hline 1 & $\begin{array}{l}\text { Correct } \\
\text { Add/Sub }\end{array}$ & $\begin{array}{l}\text { Operation is }+ \text { or }- \\
\text { and denominators of } \\
\text { operands are equal }\end{array}$ & $\begin{array}{l}\text { Create goals to set denominator of } \\
\text { answer equal to common } \\
\text { denominator, and set numerator of } \\
\text { answer equal to result of performing } \\
\text { given operation on numerators }\end{array}$ \\
\hline 2 & $\begin{array}{l}\text { Correct } \\
\text { Add/Sub }\end{array}$ & $\begin{array}{l}\text { Operation is }+ \text { or }- \\
\text { and denominators of } \\
\text { operands are } \\
\text { unequal }\end{array}$ & $\begin{array}{l}\text { Create goals to convert operands to a } \\
\text { common denominator, then set } \\
\text { denominator of answer equal to } \\
\text { common denominator, and set } \\
\text { numerator of answer equal to result of } \\
\text { performing given operation on } \\
\text { numerators }\end{array}$ \\
\hline 3 & $\begin{array}{l}\text { Correct } \\
\text { Mult }\end{array}$ & Operation is $\times$ & $\begin{array}{l}\text { Create goals to set denominator and } \\
\text { numerator of answer equal to results } \\
\text { of performing given operation on } \\
\text { operand denominators and } \\
\text { numerators respectively }\end{array}$ \\
\hline 4 & $\begin{array}{l}\text { Correct } \\
\text { Div }\end{array}$ & Operation is $\div$ & $\begin{array}{l}\text { Create goal to execute Div strategy } \\
\text { (see Table } 3 \text {, Rule } 15 \text { ) }\end{array}$ \\
\hline $5^{*}$ & $\begin{array}{l}\text { Op- } \\
\text { deleted } \\
\text { Add/Sub }\end{array}$ & $\begin{array}{l}\text { Operand } \\
\text { denominators are } \\
\text { equal }\end{array}$ & Same as Rule 1 \\
\hline $6^{*}$ & $\begin{array}{l}\text { Op- } \\
\text { deleted } \\
\text { Add/Sub }\end{array}$ & $\begin{array}{l}\text { Operand } \\
\text { denominators are } \\
\text { unequal }\end{array}$ & Same as Rule 2 \\
\hline $7 *$ & $\begin{array}{l}\text { Op- } \\
\text { deleted } \\
\text { Mult }\end{array}$ & None & Same as Rule 3 \\
\hline $8^{*}$ & $\begin{array}{l}\text { Op- } \\
\text { deleted } \\
\text { Div }\end{array}$ & None & Same as Rule 4 \\
\hline $9 *$ & $\begin{array}{l}\text { Cross- } \\
\text { Multiply }\end{array}$ & Operation is $\times$ & $\begin{array}{l}\text { Create goals to set numerator and } \\
\text { denominator of answer equal to } \\
\text { results of performing given operation } \\
\text { on operand numerators and } \\
\text { denominators cross-wise }\end{array}$ \\
\hline
\end{tabular}


Execution rules implemented in FARRA

\begin{tabular}{|c|c|c|c|}
\hline Rule & Rule Name & Condition & Action \\
\hline 10 & $\begin{array}{l}\text { Operate- } \\
\text { Numerators }\end{array}$ & $\begin{array}{l}\text { Goal exists to set } \\
\text { answer numerator to } \\
\text { result of performing } \\
\text { given operation on } \\
\text { numerators }\end{array}$ & $\begin{array}{l}\text { Create goal to solve } \\
\text { appropriate whole number } \\
\text { arithmetic problem }\end{array}$ \\
\hline 11 & $\begin{array}{l}\text { Operate- } \\
\text { Denominators }\end{array}$ & $\begin{array}{l}\text { Goal exists to set } \\
\text { answer denominator to } \\
\text { result of performing } \\
\text { given operation on } \\
\text { denominators }\end{array}$ & $\begin{array}{l}\text { Create goal to solve } \\
\text { appropriate whole number } \\
\text { arithmetic problem }\end{array}$ \\
\hline 12 & $\begin{array}{l}\text { Maintain- } \\
\text { Common- } \\
\text { Denominator }\end{array}$ & $\begin{array}{l}\text { Goal exists to set } \\
\text { answer denominator to } \\
\text { common denominator } \\
\text { of operands }\end{array}$ & $\begin{array}{l}\text { Set answer denominator equal } \\
\text { to common denominator of } \\
\text { operands }\end{array}$ \\
\hline 13 & $\begin{array}{l}\text { Convert- } \\
\text { Common- } \\
\text { Denom-By-LCD }\end{array}$ & $\begin{array}{l}\text { Goal exists to convert } \\
\text { operands to common } \\
\text { denominator }\end{array}$ & $\begin{array}{l}\text { Create goals to convert both } \\
\text { operands using the LCD } \\
\text { procedure }\end{array}$ \\
\hline 14 & $\begin{array}{l}\text { Convert } \\
\text { Common- } \\
\text { Denom-By- } \\
\text { Mult-Denoms }\end{array}$ & $\begin{array}{l}\text { Goal exists to convert } \\
\text { operands to common } \\
\text { denominator }\end{array}$ & $\begin{array}{l}\text { Create goals to multiply } \\
\text { numerator and denominator of } \\
\text { each operand by denominator } \\
\text { of other operand }\end{array}$ \\
\hline 15 & $\begin{array}{l}\text { Execute-Invert- } \\
\text { Operate }\end{array}$ & $\begin{array}{l}\text { Goal exists to execute } \\
\text { Div strategy }\end{array}$ & $\begin{array}{l}\text { Create goals to invert } 2^{\text {nd }} \\
\text { operand, change } \div \text { (if present) } \\
\text { to } \times \text {, then set answer } \\
\text { denominator and numerator to } \\
\text { results of performing given } \\
\text { operation on operand } \\
\text { denominators and numerators } \\
\text { respectively }\end{array}$ \\
\hline $16^{*}$ & $\begin{array}{l}\text { Convert- } \\
\text { Denoms-Only- } \\
\text { By-Mult- } \\
\text { Denoms }\end{array}$ & $\begin{array}{l}\text { Goal exists to convert } \\
\text { operands to common } \\
\text { denominator }\end{array}$ & $\begin{array}{l}\text { Create goals to multiply the } \\
\text { denominator of each operand } \\
\text { by the denominator of the } \\
\text { other operand }\end{array}$ \\
\hline $17^{*}$ & $\begin{array}{l}\text { Execute-Invert- } \\
\text { Operate-Forget- } \\
\text { Invert }\end{array}$ & $\begin{array}{l}\text { Goal exists to execute } \\
\text { Div strategy }\end{array}$ & $\begin{array}{l}\text { Create goals to change } \div \text { (if } \\
\text { present) to } \times \text {, then set answer } \\
\text { denominator and numerator to } \\
\text { results of performing given } \\
\text { operation on operand } \\
\text { denominators and numerators } \\
\text { respectively }\end{array}$ \\
\hline $18^{*}$ & $\begin{array}{l}\text { Execute-Invert- } \\
\text { Operate- } \\
\text { Random-Invert }\end{array}$ & $\begin{array}{l}\text { Goal exists to execute } \\
\text { Div strategy }\end{array}$ & $\begin{array}{l}\text { Create goals to invert one } \\
\text { operand, change } \div \text { (if present) } \\
\text { to } \times \text {, then set answer } \\
\text { denominator and numerator to } \\
\text { results of performing given } \\
\text { operation on operand } \\
\text { denominators and numerators } \\
\text { respectively }\end{array}$ \\
\hline
\end{tabular}

Rules denoted with a * are mal-rules 
APPENDIX B: MATHEMATICS BACKGROUND AND INTERESTS

\section{QUESTIONNAIRE}

1.Age: (in years)

2.Sex:malefemale

3.Current major (e.g., B.A. in Psychology; BSc in Engineering:

4. Year in Program (e.g., $1^{\text {st }}, 2^{\text {nd }}, 3^{\text {rd }}, 4^{\text {th }}, 5^{\text {th. }}$

5 (a). In which province did you receive your education (or country if not Canada) Elementary

Secondary

Post Secondary (University)

(b) What was the last grade (or equivalent course level) in which you took mathematics in high school (e.g., 12, 11, 10 or 9):

6. What languages do you speak?

English

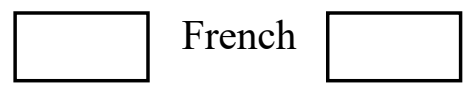

Other (please sp

7. Which do you consider your first language (i.e., the one you are the most proficient at speaking

English

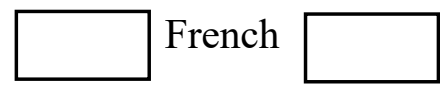

Other (please $\mathrm{sp}$

8. If you were in French immersion in school, please answer the following questions:

(a) I was in French immersion from grades to

(b) I studied math in French from grades to 


\section{MATHEMATICS}

1. When did you start using a calculator? (e.g., Grade 6, first year university):

2. When you do calculations such as $6 \times 9$, how frequently do you use a calculator

$\begin{array}{ccccc}1 & 2 & 3 & 4 & 5 \\ \text { never } & \text { rarely } & \text { sometimes } & \text { often } & \text { always }\end{array}$

3. Please rate your level of basic mathematical skill (e.g., skill at arithmetic):

$\begin{array}{ccccc}1 & 2 & 3 & 4 & 5 \\ \text { low } & & \text { moderate } & & \text { high }\end{array}$

4. Please rate your level of skill with fractions:

$\begin{array}{ccccc}1 & 2 & 3 & 4 & 5 \\ \text { low } & & \text { moderate } & & \text { high }\end{array}$

5. Please rate your level of mathematical skill in more complex areas of mathematics (e.g., calculus, algebra):

$\begin{array}{ccccc}1 & 2 & 3 & 4 & 5 \\ \text { low } & & \text { moderate } & & \text { high }\end{array}$

\section{SOLVING SIMPLE ARITHMETIC PROBLEMS}

1. How frequently do you solve math problems such as $3 \times 9$ by switching the numbers around so that the larger number is placed first $(9 \times 3)$ ?

$\begin{array}{ccccc}1 & 2 & 3 & 4 & 5 \\ \text { never } & \text { rarely } & \text { sometimes } & \text { often } & \text { always }\end{array}$

2. How frequently do you solve math problems such as $7 \times 6$ by switching the numbers around so that the smaller number is placed first $(6 \times 7)$ ?

$\begin{array}{ccccc}1 & 2 & 3 & 4 & 5 \\ \text { never } & \text { rarely } & \text { sometimes } & \text { often } & \text { always }\end{array}$




\section{SOLVING FRACTION ARITHMETIC PROBLEMS}

1. How frequently do you solve fraction multiplication problems such as $3 / 4 \times$ $5 / 6$ by switching the fractions around so that the fraction with the larger numerator is placed first $(5 / 6 \times 3 / 4)$ ?

$\begin{array}{ccccc}1 & 2 & 3 & 4 & 5 \\ \text { never } & \text { rarely } & \text { sometimes } & \text { often } & \text { always }\end{array}$

2. How frequently do you solve fraction multiplication problems such as $6 / 7 \times$ $4 / 5$ by switching the fractions around so that the fraction with the smaller numerator is placed first $(4 / 5 \times 6 / 7)$ ?

$\begin{array}{ccccc}1 & 2 & 3 & 4 & 5 \\ \text { never } & \text { rarely } & \text { sometimes } & \text { often } & \text { always }\end{array}$

3. How frequently do you solve fraction multiplication problems such as $4 / 5 \times$ $3 / 7$ by switching the fractions around so that the fraction with the larger denominator is placed first $(3 / 7 \times 4 / 5)$ ?

$\begin{array}{ccccc}1 & 2 & 3 & 4 & 5 \\ \text { never } & \text { rarely } & \text { sometimes } & \text { often } & \text { always }\end{array}$

4. How frequently do you solve fraction multiplication problems such as $3 / 8 \times$ $5 / 6$ by switching the fractions around so that the fraction with the smaller denominator is placed first $(5 / 6 \times 3 / 8)$ ?

$\begin{array}{ccccc}1 & 2 & 3 & 4 & 5 \\ \text { never } & \text { rarely } & \text { sometimes } & \text { often } & \text { always }\end{array}$




\section{APPENDIX C: FRACTION KNOWLEDGE ASSESSMENT}

Name: Age:

Please answer the following questions to the best of your ability.

1. Shade in two-thirds of each of these shapes:

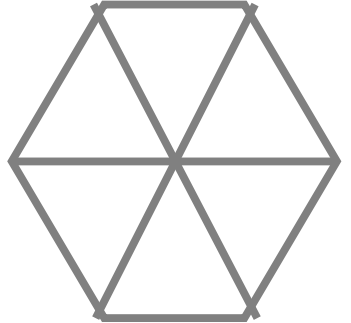

(a)

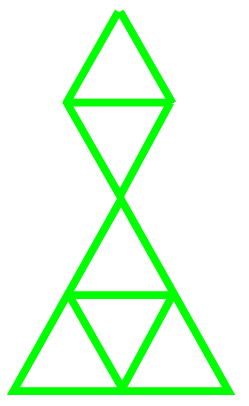

(b)

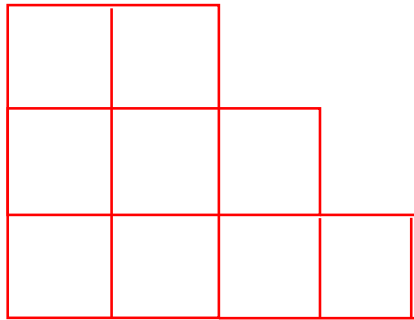

(c)

2. Please solve this problem and show your working:

$$
\frac{4}{7}+\frac{9}{14}=
$$

3. Circle the larger fraction in each pair. The first one is done for you.
(a) $\frac{1}{4}\left(\frac{3}{4}\right.$
(b) $\frac{3}{7} \frac{5}{7}$
(c) $\frac{3}{5} \quad \frac{3}{4}$
(d) $\frac{2}{5} \frac{3}{10}$
(e) $\frac{4}{5}$
$\frac{5}{6}$

4. Please solve this problem and show your working:

$$
\frac{4}{5}-\frac{5}{8}=
$$

5. Please solve this problem and show your workings:

$$
4 \frac{2}{5}+3 \frac{3}{4}=
$$


6. Mary and John both have pocket money. Mary spends $\frac{1}{4}$ of hers, while John spends $\frac{1}{2}$ of his.

(a) Is it possible for Mary to have spent more than John?

(b) Why do you think so?

7. Please solve this problem and show your workings:

$$
\frac{3}{5} \times \frac{5}{12}=
$$

8. I am tiling a floor. So far, I have tiled the shaded part of the floor diagram below.

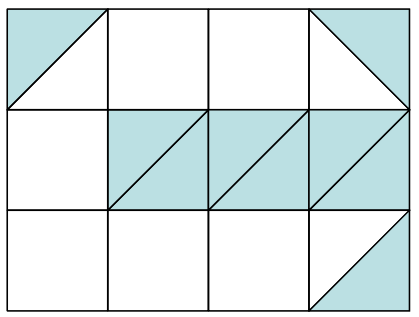

What fraction of the floor has been tiled?

9. Please solve this problem and show your workings:

$$
\frac{2}{3} \div \frac{2}{6}=
$$

10. Estimate the sum of $\frac{12}{13}+\frac{7}{8}$. Which of the following numbers is closest to this sum?

Circle your answer.
(a) 1
(b) 2
(c) 19
(d) 21 
11. Which number should go in place of the "?" in order to make these two fractions equal? Show your working:

$$
\begin{array}{ll}
6 \\
\frac{-}{9}
\end{array} \quad \frac{?}{3} \text { Answer: }
$$

12. Circle the larger fraction in each pair. The first one is done for you.
(a) $\frac{1}{4}\left(\frac{3}{4}\right)$
(b) $\frac{4}{9} \frac{2}{3}$
(c) $\frac{6}{5} \quad \frac{5}{6}$
(d) $\frac{7}{6} \quad \frac{83}{90}$
(e) $\frac{14}{13}$

\section{3}

12

13. Please solve this problem and show your working:

$$
1-\frac{5}{12}=
$$

14. Put these fractions in order of size, from the smallest on the left to the largest on the right.

$$
\begin{array}{llll}
\frac{3}{4} & \frac{2}{5} & \frac{5}{4} & \frac{2}{3}
\end{array}
$$

15. Which number should go in place of the "?" in order to make these two fractions equal? Show your workings:

$$
\frac{2}{?}=\frac{8}{28} \text { Answer: }
$$

16. How many possible fractions are between $\frac{1}{4}$ and $\frac{1}{2}$ ? 
17. Please solve this problem and show your workings:

$$
2 \times \frac{1}{8}=
$$

18. Shade in $\frac{1}{6}$ of the checkered part of the disc.

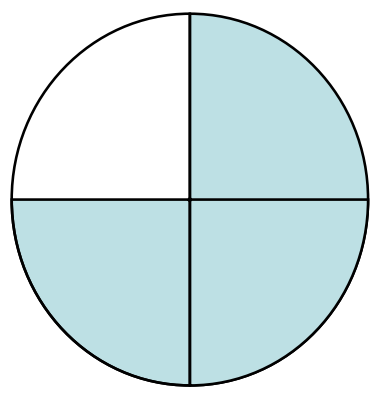

What fraction of the whole disc have you shaded?

20. Which number should go in place of the "?" in order to make these two fractions equal? Show your working:

$$
\frac{4}{5}=\frac{?}{15} \quad \text { Answer: }
$$

21. Circle EACH and EVERY fraction below that is equal to $\frac{2}{3}$.
(a) $\frac{9}{12}$
(b) $\frac{5}{6}$
(c) $\frac{8}{12}$
(d) $\frac{12}{15}$
(e) $\frac{12}{16}$
(f) $\frac{6}{9}$

22. Please solve this problem and show your workings:

$$
1 \frac{1}{5}-\frac{3}{5}=
$$

23. A relay race is run in stages of $\frac{\mathbf{1}}{\mathbf{8}} \mathrm{km}$ each. Each runner runs one stage. How many runners would be required to run a total distance of $\frac{3}{4} \mathrm{~km}$ ? 
24. Please solve this problem and show your working:

$$
\frac{3}{4} \div \frac{3}{8}=
$$


APPENDIX D: DESCRIPTIVE STATISTICS FOR PARTICIPANTS IN

STUDY 1

Descriptive statistics of participants $(\mathrm{N}=720)$ for Study 1 .

\begin{tabular}{|c|c|c|}
\hline & No of Observations & $(\%)$ \\
\hline \multicolumn{3}{|c|}{ Country of Elementary school education } \\
\hline Canada & 558 & 77.5 \\
\hline China & 47 & 6.5 \\
\hline Others & 115 & 16.0 \\
\hline \multicolumn{3}{|l|}{ Country of High school education } \\
\hline Canada & 603 & 88.8 \\
\hline Others & 48 & 16.3 \\
\hline \multicolumn{3}{|l|}{ First Language } \\
\hline English & 559 & 77.6 \\
\hline Chinese/Mandarin/Cantonese & 53 & 7.4 \\
\hline Arabic & 30 & 4.2 \\
\hline French & 20 & 2.8 \\
\hline Others & 58 & 8.1 \\
\hline \multicolumn{3}{|c|}{ Level of last mathematics course in High } \\
\hline \multicolumn{3}{|l|}{ School } \\
\hline Grade 9 & 2 & 0.3 \\
\hline Grade 10 & 15 & 2.1 \\
\hline Grade 11 & 106 & 14.7 \\
\hline Grade 12 & 552 & 76.7 \\
\hline Grade 13 & 44 & 6.1 \\
\hline "no answer" & 1 & 0.1 \\
\hline
\end{tabular}




\section{APPENDIX E: DESCRIPTIVES FOR MBIQ}

Percentage of procedural and conceptual solvers" responses to the question "When did you start using a calculator?

$\begin{array}{cc}\text { procedurally- } & \text { conceptually- } \\ \text { stronger solvers } & \text { stronger solvers } \\ (n=346) & (n=374)\end{array}$

When did you start using a calculator?

$\begin{array}{lcc}1 \text { st to } 4^{\text {th }} \text { grade } & 19.3 & 23.8 \\ 5 \text { th to } 8^{\text {th }} \text { grade } & 48.6 & 54.3 \\ 9 \text { th to } 12^{\text {th }} \text { grade } & 23.1 & 16.6 \\ \text { University } & 5.8 & 1.1 \\ \text { Others } & 3.2 & 4.3\end{array}$




\section{APPENDIX F: INFERENTIAL STATISTICS FOR MBIQ}

Table F.1. Mean responses (and standard deviations) of proc. (procedurally-stronger) and conc. (Conceptually-stronger) solvers on the mathematics, solving simple arithmetic problems and solving fraction arithmetic problems sections of the MBIQ.

Responses were provided on a Likert scale from 1(never) to 5(always)

\begin{tabular}{|c|c|c|c|c|c|c|c|c|}
\hline & \multicolumn{2}{|c|}{ proc. $(n=346)$} & \multicolumn{2}{|c|}{ conc. $(n=374)$} & \multirow[t]{2}{*}{$d f$} & \multirow[t]{2}{*}{$t$} & \multirow[t]{2}{*}{$p$} & \multirow[t]{2}{*}{ Cohen's d } \\
\hline Mathematics Skill & $M$ & $S D$ & $M$ & $S D$ & & & & \\
\hline $\begin{array}{l}\text { - When you do calculations such as } 6 \times 9 \text {, how } \\
\text { frequently do you use a calculator }\end{array}$ & 2.5 & 1.4 & 2.6 & 1.3 & 717 & .74 & .46 & 0.07 \\
\hline $\begin{array}{l}\text { - Please rate your level of basic mathematical } \\
\text { skill (e.g., skill at arithmetic) }\end{array}$ & 4.1 & 1.0 & 4.0 & 0.9 & 714 & $2.17 *$ & .03 & 0.11 \\
\hline - Please rate your level of skill with fractions: & 3.2 & 1.1 & 2.9 & 1.2 & 708 & $3.80 * *$ & $<.005$ & 0.26 \\
\hline $\begin{array}{l}\text { - Please rate your level of mathematical skill } \\
\text { in more complex areas of mathematics (e.g., } \\
\text { calculus, algebra): }\end{array}$ & 3.6 & 1.1 & 3.4 & 1.1 & 705 & $2.79 * *$ & $<.005$ & 0.18 \\
\hline \multicolumn{9}{|l|}{ Solving simple arithmetic problems } \\
\hline $\begin{array}{l}\text { How frequently do you solve math problems } \\
\text { such as } 3 \times 9 \text { by switching the numbers } \\
\text { around so that the larger number is placed } \\
\text { first }(9 \times 3) \text { ? }\end{array}$ & 2.3 & 1.5 & 2.2 & 1.4 & 713 & .47 & .64 & 0.07 \\
\hline $\begin{array}{l}\text { How frequently do you solve math problems } \\
\text { such as } 7 \times 6 \text { by switching the numbers } \\
\text { around so that the smaller number is placed } \\
\text { first }(6 \times 7)\end{array}$ & 2.3 & 1.4 & 2.2 & 1.3 & 712 & .58 & .56 & 0.07 \\
\hline
\end{tabular}




\begin{tabular}{|c|c|c|c|c|c|c|c|c|}
\hline Solving fraction arithmetic problems & & & & & & & & \\
\hline $\begin{array}{l}\text { - How frequently do you solve fraction } \\
\text { multiplication problems such as } 3 / 4 \times 5 / 6 \text { by } \\
\text { switching the fractions around so that the } \\
\text { fraction with the larger numerator is placed } \\
\text { first }(5 / 6 \times 3 / 4) \text { ? }\end{array}$ & 2.0 & 1.3 & 2.0 & 1.2 & 717 & .18 & .86 & 0 \\
\hline $\begin{array}{l}\text { How frequently do you solve fraction } \\
\text { multiplication problems such as } 6 / 7 \times 4 / 5 \text { by } \\
\text { around so that the fraction with the smaller } \\
\text { numerator is placed first }(4 / 5 \times 6 / 7) \text { ? }\end{array}$ & 2.1 & 1.3 & 2.1 & 1.2 & 716 & .49 & .63 & 0 \\
\hline $\begin{array}{l}\text { - How frequently do you solve fraction } \\
\text { multiplication problems such as } 4 / 5 \times 3 / 7 \text { by } \\
\text { switching the fractions around so that the } \\
\text { fraction with the larger denominator is } \\
\text { placed first }(3 / 7 \times 4 / 5) \text { ? }\end{array}$ & 2.1 & 1.3 & 2.1 & 1.3 & 717 & .04 & .97 & 0 \\
\hline $\begin{array}{l}\text { - How frequently do you solve fraction } \\
\text { multiplication problems such as } 3 / 8 \times 5 / 6 \text { by } \\
\text { switching around so that the fraction with the } \\
\text { smaller denominator is placed first }(5 / 6 \times \\
3 / 8) \text { ? }\end{array}$ & 2.2 & 1. & 2.1 & 1.2 & 717 & .69 & .49 & 0.01 \\
\hline
\end{tabular}

${ }^{*} \mathrm{p}<.05,{ }^{*} \mathrm{p}<.005$ 


\section{APPENDIX G: RESPONSES FOR FARRA PROBLEMS IN STUDY 1}

Table G.1. Frequency of common responses (and percentages) for same denominator fraction addition FARRA problems in Study 1.

\begin{tabular}{|c|c|c|c|c|c|c|c|c|c|}
\hline & \multicolumn{3}{|c|}{$3 / 5+1 / 5$} & \multicolumn{3}{|c|}{$3 / 5+2 / 5$} & \multicolumn{3}{|c|}{$4 / 5+3 / 5$} \\
\hline & response & $\mathrm{n}$ & $\%$ & response & $\mathrm{n}$ & $\%$ & response & $\mathrm{n}$ & $\%$ \\
\hline \multicolumn{10}{|l|}{ Correct } \\
\hline - reduced to simplest fraction & $4 / 5$ & 534 & 78.9 & 1 & 536 & 79.2 & $7 / 5$ & 450 & 66.5 \\
\hline - decimal & 0.8 & 35 & 5.2 & & & & 1.4 & 106 & 15.7 \\
\hline \multirow[t]{2}{*}{ - not reduced to simplest fraction } & $20 / 25$ & 8 & 1.2 & $5 / 5$ & 28 & 4.1 & $35 / 25$ & 7 & 1.0 \\
\hline & $8 / 10$ & 2 & 0.3 & $1 / 1$ & 5 & 0.7 & & & \\
\hline Total (in \%) & & 579 & 85.6 & & 569 & 84.0 & & 563 & 83.2 \\
\hline \multicolumn{10}{|l|}{ Independent whole number } \\
\hline - not reduced to simplest fraction & $4 / 10$ & 35 & 5.2 & $5 / 10$ & 15 & 2.2 & & & \\
\hline \multirow{2}{*}{$\begin{array}{ll}\text { - } & \text { reduced to simplest fraction } \\
& \text { Total (in \%) } \\
\end{array}$} & $2 / 5$ & 9 & 1.3 & $1 / 2$ & 18 & 2.7 & $7 / 10$ & 39 & 5.8 \\
\hline & & 44 & 6.5 & & 33 & 4.9 & & 39 & 5.8 \\
\hline \multicolumn{10}{|l|}{ Wrong operation strategy } \\
\hline $\begin{array}{l}\text { - multiply numerator, maintain } \\
\text { denominator }\end{array}$ & $3 / 5$ & 3 & 0.4 & $6 / 5$ & 12 & 1.8 & $12 / 5$ & 7 & 1.0 \\
\hline Total in (\%) & & 3 & 0.4 & & 12 & 1.8 & & 7 & \\
\hline \multicolumn{10}{|l|}{ Others } \\
\hline \multirow{5}{*}{$\begin{array}{l}\text { - } \quad \text { whole number } \\
\text { - decimal } \\
\text { Total in } \mathbf{( \% )}\end{array}$} & $4 / 25$ & 2 & 0.3 & $7 / 5$ & 8 & 1.2 & $8 / 5$ & 9 & \\
\hline & & & & $6 / 25$ & 3 & 0.4 & $9 / 5$ & 4 & \\
\hline & 4 & 5 & 0.7 & 5 & 4 & 0.6 & 7 & 6 & \\
\hline & & & & & & & 1.2 & 3 & \\
\hline & & 7 & 1.0 & & 15 & 2.2 & & 22 & 3.2 \\
\hline Blank & " " & 14 & 2.1 & " " & 13 & 1.9 & " " & 8 & 1.2 \\
\hline TOTAL (\%) & & & 95.6 & & & 94.8 & & & 94.4 \\
\hline
\end{tabular}


Table G.2. Frequency of common responses (and percentages) for different denominator fraction addition FARRA problems in Study 1.

\begin{tabular}{|c|c|c|c|c|c|c|c|c|c|}
\hline & \multicolumn{3}{|c|}{$3 / 5+1 / 2$} & \multicolumn{3}{|c|}{$3 / 5+1 / 4$} & \multicolumn{3}{|c|}{$2 / 3+3 / 5$} \\
\hline & response & $\mathrm{n}$ & $\%$ & response & $\mathrm{n}$ & $\%$ & response & $\mathrm{n}$ & $\%$ \\
\hline \multicolumn{10}{|l|}{ Correct } \\
\hline - reduced to simplest fraction & $11 / 10$ & 387 & 58.2 & $17 / 20$ & 440 & 65.0 & $19 / 15$ & 386 & 57.0 \\
\hline - decimal & 1.1 & 84 & 12.4 & 0.85 & 40 & 5.9 & 1.27 & 63 & 9.3 \\
\hline Total (in \%) & & 471 & 70.6 & & 480 & 70.9 & & 449 & 66.3 \\
\hline \multicolumn{10}{|l|}{ Independent whole number } \\
\hline - reduced to simplest fraction & $4 / 7$ & 47 & 6.9 & $4 / 9$ & 50 & 7.4 & $5 / 8$ & 43 & 6.4 \\
\hline Total (in \%) & & 47 & 6.9 & & 50 & 7.4 & & 43 & 6.4 \\
\hline \multicolumn{10}{|l|}{ Others } \\
\hline - subtract fractions & $1 / 10$ & 6 & 0.9 & $7 / 20$ & 8 & 1.2 & $1 / 15$ & 5 & 0.7 \\
\hline - multiply fractions & $4 / 10$ & 9 & 1.3 & $4 / 20$ & 7 & 1.0 & & & \\
\hline & & & & $1 / 5$ & 3 & 0.4 & & & \\
\hline - fraction & $4 / 5$ & 5 & 0.7 & $13 / 20$ & 5 & 0.7 & $16 / 15$ & 17 & 2.5 \\
\hline & $9 / 10$ & 5 & 0.7 & $3 / 9$ & 4 & 0.6 & $5 / 15$ & 9 & 1.3 \\
\hline - whole number & 2 & 5 & 0.7 & 1 & 7 & 1.0 & 1 & 9 & 1.3 \\
\hline - decimal & 1.2 & 5 & 0.7 & & & & 1.2 & 9 & 1.3 \\
\hline Total in (\%) & & 35 & 5.2 & & 34 & 4.9 & & 49 & 7.2 \\
\hline Blank & “" & 25 & 3.7 & “ ” & 35 & 5.2 & “" & 31 & 4.6 \\
\hline TOTAL (\%) & & & 85.4 & & & 88.5 & & & 84.5 \\
\hline
\end{tabular}


Table G.3. Frequency of common responses (and percentages) for same denominator fraction subtraction FARRA problems in Study 1.

\begin{tabular}{|c|c|c|c|c|c|c|c|c|c|}
\hline & \multicolumn{3}{|c|}{$3 / 5-1 / 5$} & \multicolumn{3}{|c|}{$3 / 5-2 / 5$} & \multicolumn{3}{|c|}{$4 / 5-3 / 5$} \\
\hline & response & $\mathrm{n}$ & $\%$ & response & $\mathrm{n}$ & $\%$ & response & $\mathrm{n}$ & $\%$ \\
\hline \multicolumn{10}{|l|}{ Correct } \\
\hline - reduced to simplest fraction & $2 / 5$ & 544 & 80.3 & $1 / 5$ & 548 & 80.9 & $1 / 5$ & 556 & 82.1 \\
\hline - decimal & 0.4 & 30 & 4.4 & 0.2 & 31 & 4.6 & 0.2 & 32 & 4.7 \\
\hline - not reduced to simplest fraction & $10 / 25$ & 6 & 0.9 & $5 / 25$ & 6 & 0.9 & $5 / 25$ & 5 & 0.7 \\
\hline Total (in \%) & & 580 & 85.6 & & 585 & 86.4 & & 593 & 87.5 \\
\hline \multicolumn{10}{|l|}{ Independent whole number } \\
\hline - reduced to simplest fraction & & & & $1 / 0$ & 4 & 0.6 & $1 / 0$ & 3 & 0.4 \\
\hline Total (in \%) & & & & & 4 & 0.6 & & 3 & 0.4 \\
\hline \multicolumn{10}{|l|}{ Others } \\
\hline - add fractions & $4 / 5$ & 15 & 2.2 & & & & & & \\
\hline \multirow{2}{*}{$\begin{array}{l}\text { - add numerator divide } \\
\text { denominator }\end{array}$} & 2 & 22 & 3.2 & 1 & 26 & 3.8 & 1 & 23 & 3.4 \\
\hline & $2 / 1$ & 5 & 0.7 & & & & $1 / 1$ & 3 & 0.4 \\
\hline - fraction & $2 / 10$ & 3 & 0.4 & $3 / 4$ & 4 & 0.6 & $2 / 5$ & 3 & 0.4 \\
\hline - decimal & 0.5 & 3 & 0.4 & $2 / 5$ & 3 & 0.4 & $-1 / 5$ & 3 & 0.4 \\
\hline \multirow[t]{2}{*}{ - whole number } & 0 & 3 & 0.4 & 0 & 5 & 0.7 & 0 & 4 & 0.6 \\
\hline & & & & 3 & 3 & 0.4 & & & \\
\hline Total in $(\%)$ & & 51 & 7.3 & & 41 & 5.9 & & 36 & 5.2 \\
\hline Blank & “" & 19 & 2.8 & " " & 21 & 3.1 & “" & 15 & 2.2 \\
\hline TOTAL (\%) & & & 96.0 & & & 96.2 & & & 95.6 \\
\hline
\end{tabular}


Table G.4. Frequency of common responses (and percentages) for different denominator fraction subtraction FARRA problems in Study 1.

\begin{tabular}{|c|c|c|c|c|c|c|c|c|c|}
\hline & \multicolumn{3}{|c|}{$3 / 5-1 / 2$} & \multicolumn{3}{|c|}{$3 / 5-1 / 4$} & \multicolumn{3}{|c|}{$2 / 3-3 / 5$} \\
\hline & response & $\mathrm{n}$ & $\%$ & response & $\mathrm{n}$ & $\%$ & response & $\mathrm{n}$ & $\%$ \\
\hline \multicolumn{10}{|l|}{ Correct } \\
\hline - reduced to simplest fraction & $1 / 10$ & 437 & 64.5 & $7 / 20$ & 431 & 63.7 & $1 / 15$ & 425 & 62.8 \\
\hline - decimal & 0.1 & 43 & 6.4 & 0.35 & 41 & 6.1 & 0.07 & 14 & 2.1 \\
\hline Total (in \%) & & 480 & 70.9 & & 472 & 69.8 & & 439 & 64.9 \\
\hline \multicolumn{10}{|l|}{ Independent whole number } \\
\hline - not reduced to simplest fraction & & & & $2 / 1$ & 38 & 5.6 & $-1 /-2$ & 8 & 1.2 \\
\hline - reduced to simplest fraction & $2 / 3$ & 56 & 8.3 & 2 & 13 & 1.9 & $1 / 2$ & 18 & 2.7 \\
\hline Total (in \%) & & 56 & 8.3 & & 51 & 7.5 & & 26 & 3.9 \\
\hline \multicolumn{10}{|l|}{ Others } \\
\hline - add fractions & $11 / 10$ & 6 & 0.9 & $17 / 20$ & 4 & 0.6 & & & \\
\hline - & 1.1 & 4 & 0.6 & & & & & & \\
\hline - fraction & $1 / 5$ & 15 & 2.2 & $8 / 20$ & 6 & 0.9 & $1 / 10$ & 13 & 1.9 \\
\hline - & $1 / 2$ & 7 & 1.0 & $2 / 5$ & 4 & 0.6 & $1 / 5$ & 12 & 1.8 \\
\hline$\bullet$ & $2 / 5$ & 6 & 0.9 & $1 / 10$ & 4 & 0.6 & $4 / 15$ & 10 & 1.5 \\
\hline - whole number & 1 & 5 & 0.7 & 1 & 5 & 0.7 & & & \\
\hline - decimal & & & & & & & 0.06 & 16 & 2.4 \\
\hline Total in (\%) & & 43 & 6.3 & & 23 & 3.4 & & 51 & 8.6 \\
\hline Blank & “ ” & 39 & 5.8 & “" & 49 & 7.2 & " " & 42 & 6.2 \\
\hline TOTAL (\%) & & & 91.3 & & & 87.9 & & & 83.6 \\
\hline
\end{tabular}


Table G.5. Frequency of common responses (and percentages) for same denominator fraction multiplication FARRA problems in Study 1.

\begin{tabular}{|c|c|c|c|c|c|c|c|c|c|}
\hline & \multicolumn{3}{|c|}{$3 / 5 \times 1 / 5$} & \multicolumn{3}{|c|}{$3 / 5 \times 2 / 5$} & \multicolumn{3}{|c|}{$4 / 5 \times 3 / 5$} \\
\hline & response & $\mathrm{n}$ & $\%$ & response & $\mathrm{n}$ & $\%$ & response & $\mathrm{n}$ & $\%$ \\
\hline \multicolumn{10}{|l|}{ Correct } \\
\hline - reduced to simplest fraction & $3 / 25$ & 463 & 68.4 & $6 / 25$ & 466 & 68.8 & $12 / 25$ & 488 & 72.1 \\
\hline - decimal & 0.12 & 18 & 2.7 & 0.24 & 23 & 3.4 & 0.48 & 21 & 3.1 \\
\hline Total (in \%) & & 481 & 71.1 & & 489 & 72.2 & & 509 & 75.2 \\
\hline \multicolumn{10}{|l|}{ Wrong Fraction Operation } \\
\hline & $3 / 5$ & 64 & 9.5 & $6 / 5$ & 40 & 7.6 & $12 / 5$ & 38 & 5.6 \\
\hline & & & & $11 / 5$ & 14 & 2.0 & $22 / 5$ & 13 & 1.9 \\
\hline Total (in \%) & & 64 & 9.5 & & 54 & 9.6 & & 51 & 7.5 \\
\hline \multicolumn{10}{|l|}{ Wrong inversion } \\
\hline - not reduced to simplest fraction & $5 / 15$ & 18 & 2.7 & $10 / 15$ & 21 & 4.0 & & & \\
\hline - reduced to simplest fraction & $1 / 3$ & 6 & 0.9 & & & & $15 / 20$ & 10 & 1.5 \\
\hline Total (in \%) & & 24 & 3.6 & & 21 & 4.0 & & 10 & 1.5 \\
\hline \multicolumn{10}{|l|}{ Others } \\
\hline - add fractions & $4 / 5$ & 4 & 0.6 & & & & & & \\
\hline - subtract fractions & & & & $1 / 5$ & 8 & 1.1 & & & \\
\hline - divide fractions & 3 & 11 & 1.6 & & & & $20 / 15$ & 11 & 1.6 \\
\hline - fraction & $4 / 25$ & 18 & 2.7 & $2 / 5$ & 7 & 1.7 & $4 / 5$ & 6 & 0.9 \\
\hline - & $3 / 15$ & 4 & 0.6 & $6 / 15$ & 6 & 0.8 & $12 / 15$ & 6 & 0.9 \\
\hline - whole number & & & & & & & 1 & 6 & 0.9 \\
\hline Total in $(\%)$ & & 37 & 5.5 & & 21 & 3.6 & & 29 & 4.3 \\
\hline Blank & " " & 24 & 3.5 & " " & 27 & 4.0 & " " & 30 & 4.4 \\
\hline TOTAL (\%) & & & 93.1 & & & 89.2 & & & 92.9 \\
\hline
\end{tabular}


Table G.6. Frequency of common responses (and percentages) for different denominator fraction multiplication FARRA problems in Study 1.

\begin{tabular}{|c|c|c|c|c|c|c|c|c|c|}
\hline & \multicolumn{3}{|c|}{$3 / 5 \times 1 / 2$} & \multicolumn{3}{|c|}{$3 / 5 \times 1 / 4$} & \multicolumn{3}{|c|}{$2 / 3 \times 3 / 5$} \\
\hline & response & $\mathrm{n}$ & $\%$ & response & $\mathrm{n}$ & $\%$ & response & $\mathrm{n}$ & $\%$ \\
\hline \multicolumn{10}{|l|}{ Correct } \\
\hline - reduced to simplest fraction & $3 / 10$ & 459 & 67.8 & $3 / 20$ & 476 & 70.3 & $2 / 5$ & 143 & 21.1 \\
\hline - $\quad$ not reduced to simplest fraction & & & & & & & $6 / 15$ & 346 & 51.1 \\
\hline - decimal & 0.3 & 32 & 4.7 & 0.15 & 26 & 3.8 & 0.4 & 21 & 3.1 \\
\hline Total (in \%) & & 491 & 72.5 & & 502 & 74.1 & & 510 & 75.3 \\
\hline \multicolumn{10}{|l|}{ Wrong Fraction Operation } \\
\hline - $\quad$ not reduced & 3 & 19 & 2.8 & 3 & 16 & 2.4 & $90 / 15$ & 8 & 1.2 \\
\hline - reduced & & & & & & & 6 & 6 & 0.9 \\
\hline Total (in \%) & & 19 & 2.8 & & 16 & 2.4 & & 14 & 2.1 \\
\hline \multicolumn{10}{|l|}{ Wrong inversion } \\
\hline & $5 / 6$ & 17 & 2.5 & $5 / 12$ & 21 & 3.1 & $9 / 10$ & 18 & 2.7 \\
\hline Total (in \%) & & 17 & 2.5 & & 21 & 3.1 & & 18 & 2.7 \\
\hline \multicolumn{10}{|l|}{ Others } \\
\hline - $\quad$ subtract fractions & $1 / 10$ & 9 & 1.3 & & & & & & \\
\hline - divide fractions & $6 / 5$ & 11 & 1.6 & $12 / 5$ & 11 & 1.6 & $10 / 9$ & 15 & 2.2 \\
\hline \multirow{2}{*}{$\begin{array}{l}\text { - add numerator multiply } \\
\text { denominator }\end{array}$} & $4 / 10$ & 18 & 2.7 & $4 / 20$ & 11 & 1.6 & $5 / 15$ & 7 & 1.0 \\
\hline & $2 / 5$ & 14 & 2.1 & $1 / 5$ & 9 & 1.3 & & & \\
\hline \multirow[t]{2}{*}{ - whole number } & 1 & 10 & 1.5 & 1 & 6 & 0.9 & 5 & 6 & 0.7 \\
\hline & & & & 2 & 5 & 0.7 & & & \\
\hline Total in (\%) & & 62 & 12.0 & & 42 & 8.5 & & 28 & 3.9 \\
\hline Blank & 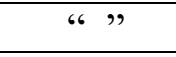 & 38 & 5.6 & 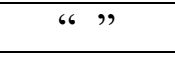 & 35 & 5.2 & ،" & 35 & 5.2 \\
\hline TOTAL (\%) & & & 92.6 & & & 91.0 & & & 89.2 \\
\hline
\end{tabular}


Table G.7. Frequency of common responses (and percentages) for same denominator fraction division FARRA problems in Study 1.

\begin{tabular}{|c|c|c|c|c|c|c|c|c|c|}
\hline & \multicolumn{3}{|c|}{$3 / 5 \div 1 / 5$} & \multicolumn{3}{|c|}{$3 / 5 \div 2 / 5$} & \multicolumn{3}{|c|}{$4 / 5 \div 3 / 5$} \\
\hline & response & $\mathrm{n}$ & $\%$ & response & $\mathrm{n}$ & $\%$ & response & $\mathrm{n}$ & $\%$ \\
\hline \multicolumn{10}{|l|}{ Correct } \\
\hline - reduced to simplest fraction & 3 & 333 & 49.2 & $3 / 2$ & 228 & 33.7 & $4 / 3$ & 236 & 34.9 \\
\hline - decimal & & & & 1.5 & 59 & 8.7 & 1.33 & 41 & 6.0 \\
\hline \multirow{2}{*}{$\begin{array}{l}\text { - } \quad \text { not reduced to simplest fraction } \\
\text { Total (in \%) }\end{array}$} & $3 / 1$ & 19 & 2.8 & & & & $20 / 15$ & 121 & 17.9 \\
\hline & & 352 & 52.0 & & 287 & 42.4 & & 398 & 58.8 \\
\hline \multicolumn{10}{|l|}{ Wrong Fraction Operation } \\
\hline & $3 / 5$ & 53 & 7.8 & $1.5 / 5$ & 9 & 1.3 & & & \\
\hline Total (in \%) & & 53 & 7.8 & & 9 & 1.3 & & & \\
\hline \multicolumn{10}{|l|}{ Wrong inversion } \\
\hline - not reduced to simplest fraction & $5 / 15$ & 84 & 12.4 & $10 / 15$ & 131 & 19.4 & $15 / 20$ & 8 & 1.2 \\
\hline - reduced to simplest fraction & $1 / 3$ & 7 & 1.0 & $2 / 3$ & 13 & 1.9 & $3 / 4$ & 13 & 1.9 \\
\hline Total (in \%) & & 91 & 13.4 & & 144 & 21.3 & & 21 & 3.1 \\
\hline \multicolumn{10}{|l|}{ Others } \\
\hline - add fractions & $4 / 5$ & 11 & 1.6 & 1 & 20 & 3.0 & $7 / 5$ & 9 & 1.3 \\
\hline - subtract fractions & $2 / 5$ & 9 & 1.3 & $1 / 5$ & 20 & 3.0 & $1 / 5$ & 25 & 3.7 \\
\hline - multiply fractions & $3 / 25$ & 17 & 2.5 & $6 / 25$ & 12 & 1.8 & $12 / 25$ & 16 & 2.4 \\
\hline & 0.12 & 7 & 1.0 & & & & & & \\
\hline - fraction & & & & $3 / 5$ & 11 & 1.6 & & & \\
\hline whole number & & & & & & & 2 & 9 & 1.3 \\
\hline Total in (\%) & & 44 & 6.4 & & 63 & 9.4 & & 59 & 8.7 \\
\hline Blank & " " & 61 & 9.0 & " " & 78 & 11.5 & " " & 84 & 12.4 \\
\hline TOTAL (\%) & & & 87.7 & & & 85.8 & & & 83.0 \\
\hline
\end{tabular}


Table G.8. Frequency of common responses (and percentages) for different denominator fraction division FARRA problems in Study 1.

\begin{tabular}{|c|c|c|c|c|c|c|c|c|c|}
\hline & \multicolumn{3}{|c|}{$3 / 5 \div 1 / 2$} & \multicolumn{3}{|c|}{$3 / 5 \div 1 / 4$} & \multicolumn{3}{|c|}{$2 / 3 \div 3 / 5$} \\
\hline & response & $\mathrm{n}$ & $\%$ & response & $\mathrm{n}$ & $\%$ & response & $\mathrm{n}$ & $\%$ \\
\hline \multicolumn{10}{|l|}{ Correct } \\
\hline - reduced to simplest fraction & $6 / 5$ & 337 & 49.8 & $12 / 5$ & 352 & 52.0 & $10 / 9$ & 329 & 48.6 \\
\hline - decimal & 1.2 & 54 & 8.0 & 2.4 & 47 & 6.9 & 1.11 & 41 & 6.1 \\
\hline Total (in \%) & & 391 & 57.8 & & & 58.9 & & & 54.7 \\
\hline \multicolumn{10}{|l|}{ Independent whole number } \\
\hline & $3 / 2.5$ & 10 & 1.5 & & & & & & \\
\hline Total (in \%) & & 10 & 1.5 & & & & & & \\
\hline \multicolumn{10}{|l|}{ Wrong Inversion } \\
\hline - Reduced to simplest fraction & $5 / 6$ & 13 & 1.9 & $5 / 12$ & 8 & 1.2 & $9 / 10$ & 20 & 3.0 \\
\hline Total (in \%) & & 13 & 1.9 & & 8 & 1.2 & & 20 & 3.0 \\
\hline \multicolumn{10}{|l|}{ Others } \\
\hline - add fractions & $11 / 10$ & 11 & 1.6 & $17 / 20$ & 10 & 1.5 & $19 / 15$ & 9 & 1.3 \\
\hline - subtract fractions & $1 / 10$ & 9 & 1.3 & & & & & & \\
\hline - multiply fractions & $3 / 10$ & 28 & 4.1 & $3 / 20$ & 25 & 3.7 & $6 / 15$ & 12 & 1.8 \\
\hline & 0.3 & 7 & 1.0 & 0.15 & 8 & 1.2 & $2 / 5$ & 9 & 1.3 \\
\hline - fraction & $3 / 5$ & 9 & 1.3 & $3 / 5$ & 6 & 0.9 & $2 / 3$ & 12 & 1.8 \\
\hline - whole number & & & & 3 & 10 & 1.5 & 1 & 7 & 1.0 \\
\hline & & & & 2 & 6 & 0.9 & & & \\
\hline - decimal & & & & & & & 0.04 & 7 & 1.0 \\
\hline Total in (\%) & & 64 & 9.3 & & 65 & 9.7 & & 56 & 8.2 \\
\hline Blank & “" & 90 & 13.3 & “ ” & 95 & 14.0 & “ ” & 100 & 14.8 \\
\hline TOTAL (\%) & & & 83.9 & & & 83.8 & & & 80.6 \\
\hline
\end{tabular}




\section{APPENDIX H: RESPONSES FOR FRACTION ARITHMETIC PROBLEMS IN STUDY 2 - PART ONE}

Table H.1: Common responses for same denominator fraction addition for procedurally-and conceptually-stronger solvers. The entry in each cell displays the percentage within each group of solvers that provided the corresponding response. Proc. Stands for procedurally-stronger solvers ( $n=31)$, Conc. Stands for conceptually-stronger solvers $(n=28)$.

\begin{tabular}{|c|c|c|c|c|c|c|c|c|c|}
\hline & \multicolumn{3}{|c|}{$3 / 10+2 / 10$} & \multicolumn{3}{|c|}{$3 / 15+2 / 15$} & \multicolumn{3}{|c|}{$4 / 20+3 / 20$} \\
\hline Identified strategy & Response & Proc. $(\%)$ & Conc. $(\%)$ & Response & Proc. (\%) & Conc. (\%) & Response & Proc. (\%) & Conc. (\%) \\
\hline \multicolumn{10}{|l|}{ Correct } \\
\hline - reduced & $1 / 2$ & 66.7 & 75 & $1 / 3$ & 81.5 & 78.1 & $7 / 20$ & 88.9 & 96.9 \\
\hline - non-reduced & $5 / 10$ & 22.2 & 18.8 & $5 / 15$ & 14.9 & 18.8 & $\mathrm{n} / \mathrm{a}$ & & \\
\hline Total (in \%) & & 88.9 & 93.8 & & 96.4 & 96.9 & & 88.9 & 96.9 \\
\hline \multicolumn{10}{|c|}{ Independent whole-number } \\
\hline - reduced & $1 / 4$ & 3.7 & 3.1 & & & & $7 / 40$ & 7.4 & 3.1 \\
\hline - non-reduced & $5 / 20$ & 3.7 & 3.1 & $5 / 30$ & 3.7 & 3.1 & & & \\
\hline Total (in \%) & & 7.4 & 6.2 & & 3.7 & 3.1 & & 7.4 & 3.1 \\
\hline \multicolumn{10}{|l|}{ Other errors } \\
\hline $\begin{array}{l}\text { - Computational } \\
\text { errors }\end{array}$ & $1 / 5$ & 3.7 & & & & & & & \\
\hline - Hybrid error & & & & & & & $7 / 400$ & 3.7 & \\
\hline TOTAL (\%) & & 3.7 & & & & & & 3.7 & \\
\hline
\end{tabular}


Table H.2: Common responses for different denominator fraction addition for procedurally-and conceptually-stronger solvers. The entry in each cell displays the percentage within each group of solvers that provided the corresponding response. Proc. Stands for procedurally-stronger solvers $(n=31)$, Conc. Stands for conceptually-stronger solvers $(n=28)$.

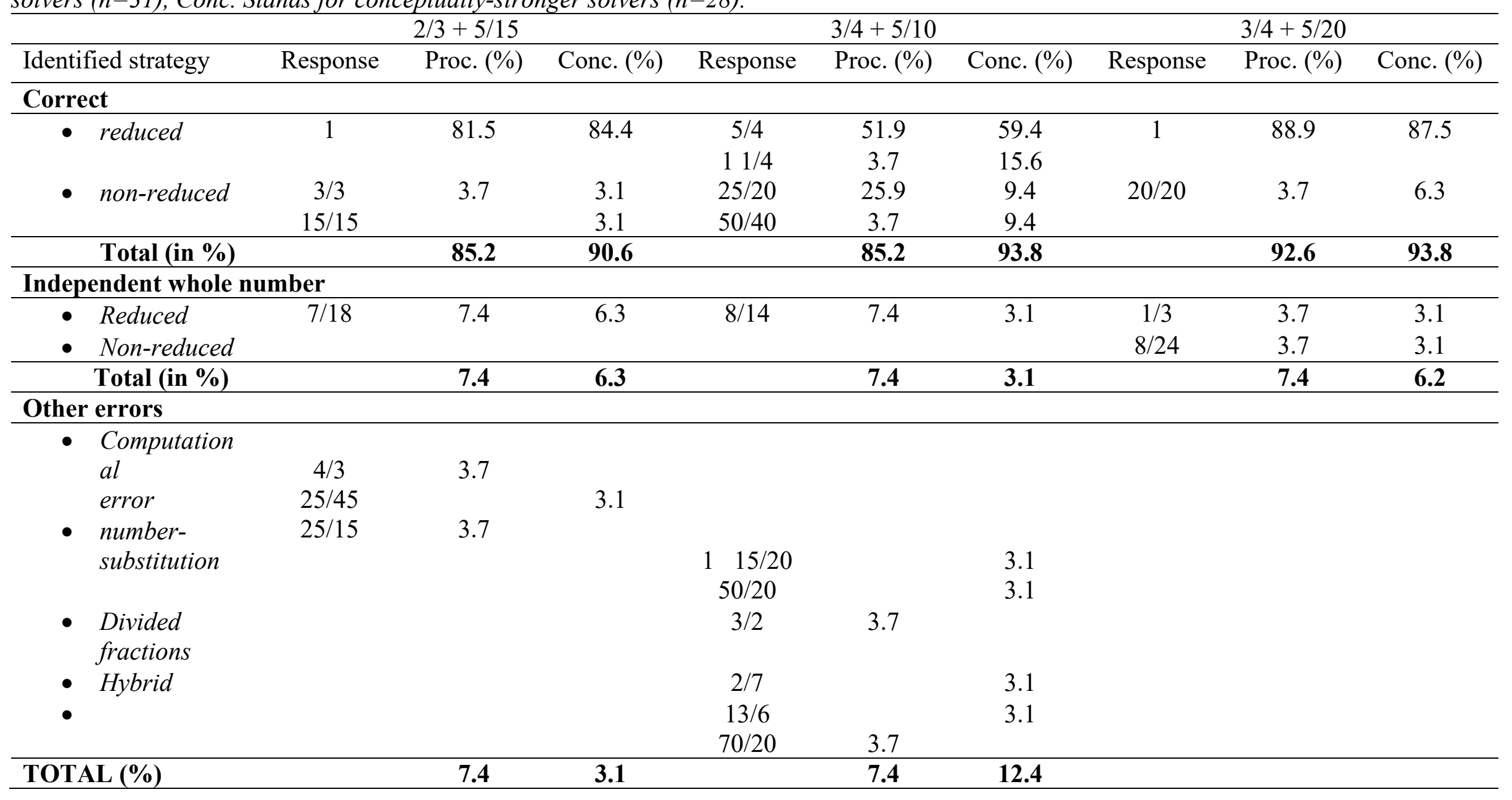


Table H.3. Common responses for same denominator fraction subtraction for procedurally-and conceptually-stronger solvers. The entry in each cell displays the percentage within each group of solvers that provided the corresponding response. Proc. Stands for procedurally-stronger solvers $(n=31)$, Conc. Stands for conceptually-stronger solvers $(n=28)$.

\begin{tabular}{|c|c|c|c|c|c|c|c|c|c|}
\hline & \multicolumn{3}{|c|}{$3 / 10-2 / 10$} & \multicolumn{3}{|c|}{$3 / 15-2 / 15$} & \multicolumn{3}{|c|}{$4 / 20-3 / 20$} \\
\hline Identified strategy & Response & Proc. $(\%)$ & Conc. $(\%)$ & Response & Proc. $(\%)$ & Conc. $(\%)$ & Response & Proc. $(\%)$ & Conc. $(\%)$ \\
\hline \multicolumn{10}{|l|}{ Correct } \\
\hline - reduced & $1 / 10$ & 92.6 & 96.9 & $1 / 15$ & 96.3 & 100 & $1 / 20$ & 96.3 & 96.9 \\
\hline - decimal & 0.1 & 3.7 & & & & & & & \\
\hline Total (in \%) & & 96.3 & 96.9 & & 96.3 & 100 & & 96.3 & 96.9 \\
\hline \multicolumn{10}{|c|}{ Independent whole number } \\
\hline & & & & & & & & & 3.1 \\
\hline Total (in \%) & & & & & & & & & 3.1 \\
\hline \multicolumn{10}{|l|}{ Other errors } \\
\hline $\begin{array}{l}\text { - other strategy } \\
\text { error }\end{array}$ & 1 & 3.7 & 3.1 & 1 & 3.7 & & 1 & 3.7 & \\
\hline TOTAL (\%) & & 3.7 & 3.1 & & 3.7 & & & 3.7 & \\
\hline
\end{tabular}


Table H.4. Common responses for different denominator fraction subtraction for procedurally-and conceptually-stronger solvers. The entry in each cell displays the percentage within each group of solvers that provided the corresponding response. Proc. Stands for procedurally-stronger solvers $(n=31)$, Conc. Stands for conceptually-stronger solvers $(n=28)$.

\begin{tabular}{|c|c|c|c|c|c|c|c|c|c|}
\hline & & $2 / 3-5 / 15$ & & & $3 / 4-5 / 10$ & & & $3 / 4-5 / 20$ & \\
\hline Identified strategy & Response & Proc. $(\%)$ & Conc. $(\%)$ & Response & Proc $(\%)$ & Conc $(\%)$ & Response & Proc. $(\%)$ & Conc $(\%)$ \\
\hline \multicolumn{10}{|l|}{ Correct } \\
\hline - reduced & $1 / 3$ & 74.1 & 75.0 & $1 / 4$ & 66.7 & 71.9 & $1 / 2$ & 81.5 & 75.0 \\
\hline \multirow[t]{3}{*}{ - non-reduced } & $5 / 15$ & 14.8 & 15.6 & $5 / 20$ & 22.2 & 3.1 & $2 / 4$ & & 3.1 \\
\hline & & & & $10 / 40$ & 3.7 & 12.5 & $10 / 20$ & 7.4 & 12.5 \\
\hline & & & & & & & $40 / 80$ & 3.7 & \\
\hline TOTAL & & 88.9 & 90.6 & & 92.6 & 86.5 & & 92.6 & 90.6 \\
\hline \multicolumn{10}{|c|}{ Independent whole number } \\
\hline - Reduced & $1 / 4$ & 3.7 & 3.1 & $1 / 3$ & 3.7 & 3.1 & $1 / 8$ & & 3.1 \\
\hline - Non-reduced & $3 / 12$ & 3.7 & & & & & $2 / 16$ & 3.7 & \\
\hline TOTAL & & 7.4 & 3.1 & & 3.7 & 3.1 & & 3.7 & 3.1 \\
\hline \multicolumn{10}{|l|}{ Other errors } \\
\hline $\begin{array}{l}\text { - Computation } \\
\text { error }\end{array}$ & $1 / 6$ & 3.7 & & & & & & & \\
\hline - number & $5 / 5$ & & 3.1 & & & & & & \\
\hline $\begin{array}{l}\text { Substumulon } \\
\text { - Addition }\end{array}$ & & & & $5 / 4$ & & 3.1 & & & \\
\hline \multirow[t]{3}{*}{ - Hybrid Error } & $-3 / 12$ & & 3.1 & $-170 / 40$ & & 3.1 & $-2 / 16$ & & 3.1 \\
\hline & & & & $-2 / 6$ & & 3.1 & $-3 / 16$ & 3.7 & \\
\hline & & & & $-3 / 6$ & 3.7 & & $-13 / 4$ & & 3.1 \\
\hline TOTAL & & 3.7 & 6.2 & & 3.7 & 9.3 & & 3.7 & 6.2 \\
\hline
\end{tabular}


Table H.5. Common responses for same denominator fraction multiplication for procedurally-and conceptually-stronger solvers. The entry in each cell displays the percentage within each group of solvers that provided the corresponding response. Proc. Stands for procedurallystronger solvers $(n=31)$, Conc. Stands for conceptually-stronger solvers $(n=28)$.

\begin{tabular}{|c|c|c|c|c|c|c|c|c|c|}
\hline & & $3 / 10 \times 2 / 10$ & & & $3 / 10 \times 5 / 10$ & & & $2 / 15 \times 5 / 15$ & \\
\hline Identified strategy & Response & Proc. $(\%)$ & Conc. (\%) & Response & Proc. $(\%)$ & Conc. $(\%)$ & Response & Proc. $(\%)$ & Conc. (\%) \\
\hline \multicolumn{10}{|l|}{ Correct } \\
\hline - Reduced & $3 / 50$ & 22.2 & 46.9 & $3 / 20$ & 37.0 & 46.9 & $2 / 45$ & 33.3 & 43.8 \\
\hline - Non-reduced & $6 / 100$ & 51.9 & 34.4 & $15 / 100$ & 51.9 & 28.1 & $10 / 225$ & 18.5 & 34.4 \\
\hline - Decimal & .06 & & 3.1 & $1.5 / 10$ & 3.7 & 3.1 & & & \\
\hline TOTAL & & 74.1 & 84.4 & & 92.6 & 78.1 & & 51.8 & 78.2 \\
\hline \multicolumn{10}{|c|}{ Wrong fraction operation } \\
\hline - Reduced & $3 / 5$ & 7.4 & 9.4 & $3 / 2$ & 3.7 & 3.1 & $2 / 3$ & 7.4 & 9.4 \\
\hline - Non-reduced & $6 / 10$ & 3.7 & & $15 / 10$ & & 3.1 & $10 / 15$ & 3.7 & 6.3 \\
\hline & & & & 1.5 & & 6.3 & & & \\
\hline TOTAL & & 11.1 & 9.4 & & 3.7 & 12.5 & & 11.1 & 15.7 \\
\hline \multicolumn{10}{|l|}{ Other errors } \\
\hline \multirow{9}{*}{$\begin{array}{l}\text { - } \begin{array}{l}\text { Computation } \\
\text { error }\end{array} \\
\end{array}$} & $1 / 25$ & 3.7 & & $3 / 10$ & & 6.3 & $10 / 125$ & 7.4 & 3.1 \\
\hline & $1 / 20$ & 3.7 & 3.1 & $3 / 40$ & & 3.1 & $10 / 1025$ & 3.7 & \\
\hline & & & & & & & $10 /(15 \times 15)$ & 3.7 & \\
\hline & & & & & & & $10 / 350$ & 3.7 & \\
\hline & & & & & & & $10 / 35$ & 3.7 & \\
\hline & & & & & & & $10 / 625$ & 3.7 & \\
\hline & & & & & & & $10 / 255$ & 3.7 & \\
\hline & & & & & & & $10 / ?$ & & 3.1 \\
\hline & & & & & & & $2 / 5$ & 3.7 & \\
\hline - Divide & $3 / 2$ & 7.4 & 3.1 & $3 / 5$ & 3.7 & & $30 / 75$ & 3.7 & 3.1 \\
\hline TOTAL & & 14.8 & 6.2 & & 3.7 & 9.4 & & 37.0 & 9.3 \\
\hline
\end{tabular}




\begin{tabular}{|c|c|c|c|c|c|c|c|c|c|}
\hline & \multicolumn{3}{|c|}{$3 / 15 \times 2 / 15$} & \multicolumn{3}{|c|}{$3 / 20 \times 5 / 20$} & \multicolumn{3}{|c|}{$4 / 20 \times 3 / 20$} \\
\hline Identified strategy & Response & Proc. $(\%)$ & Conc. $(\%)$ & Response & Proc. $(\%)$ & Conc. $(\%)$ & Response & Proc. $(\%)$ & Conc. $(\%)$ \\
\hline \multicolumn{10}{|l|}{ Correct } \\
\hline - Reduced & $2 / 75$ & 18.6 & 28.1 & $3 / 80$ & 37.0 & 50.0 & $3 / 100$ & 40.7 & 56.3 \\
\hline - Non-reduced & $6 / 225$ & 25.9 & 46.9 & $15 / 400$ & 44.4 & 28.1 & $12 / 400$ & 37.0 & 25.0 \\
\hline & & & & & & & $6 / 200$ & 3.7 & \\
\hline TOTAL & & 44.5 & 75.0 & & 81.4 & 78.1 & & 81.4 & 81.3 \\
\hline \multicolumn{10}{|c|}{ Wrong fraction operation } \\
\hline - Reduced & $2 / 5$ & 7.4 & 6.3 & $3 / 4$ & 3.7 & & $3 / 5$ & 3.7 & 3.1 \\
\hline - Non-reduced & $6 / 15$ & 3.7 & 9.4 & $15 / 20$ & 3.7 & 6.3 & $12 / 20$ & & 6.3 \\
\hline TOTAL & & 11.1 & 15.7 & & 7.4 & 12.6 & & 3.7 & 9.4 \\
\hline \multicolumn{10}{|l|}{ Others } \\
\hline - Computation & $6 / 125$ & 11.1 & 3.1 & $15 / 60$ & 3.7 & & $12 / 4000$ & 3.7 & \\
\hline error & $6 / 1025$ & 3.7 & & $3 / 60$ & 3.7 & & $16 / 40$ & 3.7 & \\
\hline & $6 / 15 \times 15$ & 3.7 & & $3 / 20$ & & 6.3 & $12 / ?$ & & 3.1 \\
\hline & $6 / 625$ & 3.7 & & $5 / 120$ & & 3.1 & $12 / 40$ & & 3.1 \\
\hline & $6 / 350$ & 3.7 & & $15 / ?$ & & 3.1 & & & \\
\hline & $6 / 175$ & 3.7 & & & & & & & \\
\hline & $6 / 35$ & 3.7 & & & & & & & \\
\hline & $10 / 225$ & 3.7 & & & & & & & \\
\hline & $6 / 150$ & & 3.1 & & & & & & \\
\hline - $\quad$ add fractions & & & & $8 / 20$ & 3.7 & & & & \\
\hline $\begin{array}{l}\text { - divide } \\
\text { fractions }\end{array}$ & $45 / 30$ & 3.7 & & & & & $4 / 3$ & 3.7 & \\
\hline - Hybrid error & $5 / 225$ & 3.7 & & & & & $4 / 5$ & & 3.1 \\
\hline & & & & & & & $2 / 5$ & 3.7 & \\
\hline - Guessed & $4 / 345$ & & 3.1 & & & & & & \\
\hline TOTAL & & 44.4 & 9.3 & & 11.1 & 12.5 & & 14.8 & 9.3 \\
\hline
\end{tabular}


Table H.6. Common responses for different denominator fraction multiplication for procedurally-and conceptually-stronger solvers. The entry in each cell displays the percentage within each group of solvers that provided the corresponding response. Proc. Stands for procedurallystronger solvers $(n=31)$, Conc. Stands for conceptually-stronger solvers $(n=28)$.

\begin{tabular}{|c|c|c|c|c|c|c|c|c|c|}
\hline & \multicolumn{3}{|c|}{$2 / 3 \times 5 / 15$} & \multicolumn{3}{|c|}{$3 / 4 \times 5 / 10$} & \multicolumn{3}{|c|}{$3 / 4 \times 5 / 20$} \\
\hline Identified strategy & Response & Proc. $(\%)$ & Conc. $(\%)$ & Response & Proc. $(\%)$ & Conc. (\%) & Response & Proc. $(\%)$ & Conc. (\%) \\
\hline \multicolumn{10}{|l|}{ Correct } \\
\hline - Reduced & $2 / 9$ & 48.1 & 50 & $3 / 8$ & 51.9 & 59.3 & $3 / 16$ & 33.3 & 50 \\
\hline - Non-reduced & $10 / 45$ & 40.7 & 34.4 & $\begin{array}{l}15 / 40 \\
1.5 / 4\end{array}$ & 44.4 & $\begin{array}{c}28.1 \\
3.1\end{array}$ & $15 / 80$ & 44.4 & 34.4 \\
\hline TOTAL & & 88.8 & 84.4 & & 96.3 & 90.5 & & 77.7 & 84.4 \\
\hline \multicolumn{10}{|c|}{ Wrong fraction operation } \\
\hline & $50 / 15$ & & 3.1 & 7.5 & & 6.3 & $75 / 20$ & & 6.3 \\
\hline & 3.33 & & 3.1 & $150 / 20$ & & 3.1 & 3.75 & & 3.1 \\
\hline TOTAL & & & 6.2 & & & 9.4 & & & 9.4 \\
\hline \multicolumn{10}{|l|}{ Others } \\
\hline - Computation & $10 / 64$ & 3.7 & & & & & $15 / 40$ & 3.7 & \\
\hline error & $1 / 6$ & & 3.1 & & & & $3 / 7$ & 3.7 & \\
\hline & $5 / 9$ & & 3.1 & & & & $3 / 8$ & 3.7 & \\
\hline & $6 / 15$ & & 3.1 & & & & $3 / 20$ & & 3.1 \\
\hline $\begin{array}{l}\text { - Add fractions } \\
\text { - Subtract }\end{array}$ & 1 & 3.7 & & & & & & & \\
\hline fractions & & & & & & & $1 / 2$ & & 3.1 \\
\hline $\begin{array}{l}\text { - Divide } \\
\text { fractions }\end{array}$ & 2 & 3.7 & & $3 / 2$ & 3.7 & & 3 & 7.4 & \\
\hline $\begin{array}{l}\text { - Hybrid } \\
\text { error }\end{array}$ & & & & & & & $15 / 4$ & 3.7 & \\
\hline TOTAL & & 11.1 & 9.4 & & 3.7 & & & 22.2 & 6.2 \\
\hline
\end{tabular}




\begin{tabular}{|c|c|c|c|c|c|c|c|c|c|}
\hline & & $5 / 3 \times 2 / 15$ & & & $5 / 4 \times 3 / 10$ & & & $5 / 4 \times 3 / 20$ & \\
\hline Identified strategy & Response & Proc. $(\%)$ & Conc. $(\%)$ & Response & Proc. $(\%)$ & Conc. $(\%)$ & Response & Proc. $(\%)$ & Conc. (\%) \\
\hline \multicolumn{10}{|l|}{ Correct } \\
\hline - Reduced & $2 / 9$ & 44.4 & 59.4 & $3 / 8$ & 40.7 & 59.4 & $3 / 16$ & 40.7 & 53.1 \\
\hline - Non-reduced & $10 / 45$ & 37.0 & 25 & $15 / 40$ & 48.1 & 31.3 & $15 / 80$ & 48.1 & 34.4 \\
\hline TOTAL & & 81.4 & 84.4 & & 88.8 & 90.6 & & 88.7 & 87.5 \\
\hline \multicolumn{10}{|c|}{ Independent whole number } \\
\hline & & & & & & & $28 / 20$ & 3.7 & \\
\hline TOTAL & & & & & & & & 3.7 & \\
\hline \multicolumn{10}{|c|}{ Wrong fraction operation } \\
\hline - non-reduced & $50 / 15$ & 3.7 & 3.1 & $150 / 20$ & & 3.1 & $75 / 20$ & & 6.3 \\
\hline - reduced & $31 / 3$ & & 6.3 & $15 / 2$ & & 6.4 & $15 / 4$ & 3.7 & \\
\hline & & & & & & & $33 / 4$ & & 3.1 \\
\hline TOTAL & & 3.7 & 9.4 & & & 9.4 & & 3.7 & 9.4 \\
\hline \multicolumn{10}{|l|}{ Others } \\
\hline $\begin{array}{l}\text { - divide } \\
\text { fractions }\end{array}$ & $25 / 2$ & 3.7 & 3.1 & & & & \multirow{6}{*}{$3 / 18$} & & \multirow{6}{*}{3.1} \\
\hline \multirow{4}{*}{$\begin{array}{l}\text { - computation } \\
\text { error }\end{array}$} & $1 / 3$ & 3.7 & 3.1 & $3 / 2$ & 3.7 & & & & \\
\hline & & & & & & & & & \\
\hline & $10 / 30$ & & 3.1 & $5 / 13.3$ & 3.7 & & & & \\
\hline & $10 / 35$ & 3.7 & & & & & & & \\
\hline hybrid & $1 / 2$ & 3.7 & & $-3 / 2$ & 3.7 & & & & \\
\hline TOTAL & & 14.8 & 6.2 & & 11.1 & & & & 3.1 \\
\hline
\end{tabular}


Table H.7. Common responses for same-denominator fraction division for procedurally-and conceptually-stronger solvers. The entry in each cell displays the percentage within each group of solvers that provided the corresponding response. Proc. Stands for procedurally-stronger solvers ( $n=31)$, Conc. Stands for conceptually-stronger solvers $(n=28)$.

\begin{tabular}{|c|c|c|c|c|c|c|c|c|c|}
\hline & \multicolumn{3}{|c|}{$3 / 10 \div 2 / 10$} & \multicolumn{3}{|c|}{$3 / 15 \div 2 / 15$} & \multicolumn{3}{|c|}{$4 / 20 \div 3 / 20$} \\
\hline Identified strategy & Response & Proc. $(\%)$ & Conc. (\%) & Response & Proc. $(\%)$ & Conc. $(\%)$ & Response & Proc. $(\%)$ & Conc. (\%) \\
\hline \multicolumn{10}{|l|}{ Correct } \\
\hline - reduced & $3 / 2$ & 63.0 & 53.1 & $3 / 2$ & 66.7 & 59.4 & $4 / 3$ & 59.3 & 62.5 \\
\hline - non-reduced & $15 / 10$ & 3.7 & 3.1 & $1.5 / 1$ & 3.7 & & $8 / 6$ & 3.7 & \\
\hline & $30 / 20$ & 18.5 & 18.8 & $45 / 30$ & 7.4 & 18.8 & $80 / 60$ & 14.8 & 18.8 \\
\hline - decimal & 1.5 & 3.7 & 6.3 & 1.5 & & 3.1 & 1.33 & & 3.1 \\
\hline TOTAL & & 88.9 & 81.3 & & 77.8 & 81.3 & & 77.8 & 84.4 \\
\hline \multicolumn{10}{|c|}{ Wrong fraction operation } \\
\hline & $\begin{array}{c}1.5 / 10 \\
3 / 20\end{array}$ & 3.7 & 3.1 & $1.5 / 15$ & 3.7 & 3.1 & $1.3 / 20$ & 3.7 & \\
\hline TOTAL & & 3.7 & 3.1 & & 3.7 & 3.1 & & 3.7 & \\
\hline \multicolumn{10}{|l|}{ Wrong inversion } \\
\hline & $2 / 3$ & & 6.3 & $2 / 3$ & & 6.3 & $3 / 4$ & & 3.15 \\
\hline & & & & & & & $6 / 8$ & & 3.15 \\
\hline TOTAL & & & 6.3 & & & 6.3 & & & 6.3 \\
\hline
\end{tabular}




\begin{tabular}{|c|c|c|c|c|c|c|c|c|c|}
\hline \multicolumn{10}{|l|}{ Others } \\
\hline - subtract & $1 / 10$ & & 6.3 & $1 / 15$ & & 3.1 & $1 / 20$ & 3.7 & 3.1 \\
\hline - computation & & & & $4 / 3$ & 3.7 & & $4 / 30$ & 3.7 & \\
\hline & & & & $45 / 36$ & 3.7 & & $40 / 60$ & 3.7 & \\
\hline - number & & & & & & & $1 / 5$ & & \\
\hline substitution & $1 / 0$ & 3.7 & & 1 & & 3.1 & & 3.7 & \\
\hline & & & & $1 / 30$ & & 3.1 & & & \\
\hline - hybrid & $1 / 20$ & & 3.1 & & & & $3.3 / 20$ & & 3.1 \\
\hline & & & & & & & $12 / 40$ & & 3.1 \\
\hline - guessed & & & & $135 / 110$ & 3.7 & & & & \\
\hline & & & & 2 & 3.7 & & & & \\
\hline - blank & “ ” & 3.7 & & ،" & 3.7 & & “ $"$ & 3.7 & \\
\hline TOTAL & & 7.4 & 9.4 & & 18.5 & 9.3 & & 18.5 & 9.3 \\
\hline
\end{tabular}


Table H.8. Common responses for different-denominator fraction division for procedurally-and conceptually-stronger solvers. The entry in each cell displays the percentage within each group of solvers that provided the corresponding response. Proc. Stands for procedurally-stronger solvers ( $n=31)$, Conc. Stands for conceptually-stronger solvers $(n=28)$.

\begin{tabular}{|c|c|c|c|c|c|c|c|c|c|}
\hline & \multicolumn{3}{|c|}{$2 / 3 \div 5 / 15$} & \multicolumn{3}{|c|}{$3 / 4 \div 5 / 10$} & \multicolumn{3}{|c|}{$3 / 4 \div 5 / 20$} \\
\hline Identified strategy & Response & Proc. $(\%)$ & Conc. $(\%)$ & Response & Proc. $(\%)$ & Conc. $(\%)$ & Response & Proc. $(\%)$ & Conc. $(\%)$ \\
\hline \multicolumn{10}{|l|}{ Correct } \\
\hline - reduced & 2 & 66.7 & 65.6 & $3 / 2$ & 55.6 & 53.1 & 3 & 63.0 & 68.8 \\
\hline $\begin{array}{l}\text { - Partially } \\
\text { reduced }\end{array}$ & $6 / 3$ & 3.7 & 3.1 & $6 / 4$ & 3.7 & 3.1 & $6 / 2$ & 3.7 & \\
\hline - nonreduced & $\begin{array}{c}10 / 5 \\
30 / 15\end{array}$ & $\begin{array}{c}3.7 \\
11.1\end{array}$ & 12.5 & $\begin{array}{c}30 / 20 \\
1.5\end{array}$ & $\begin{array}{c}22.2 \\
3.7\end{array}$ & $\begin{array}{c}18.8 \\
9.4\end{array}$ & $\begin{array}{c}12 / 4 \\
15 / 5 \\
60 / 20\end{array}$ & $\begin{array}{c}3.7 \\
11.1\end{array}$ & 3.1 \\
\hline TOTAL & & 85.2 & 81.2 & & 85.2 & 84.4 & & 81.5 & 84.4 \\
\hline \multicolumn{10}{|c|}{ Wrong fraction operation } \\
\hline & $2 / 15$ & 3.7 & 6.3 & $\begin{array}{c}1.5 / 20 \\
3 / 40 \\
\end{array}$ & & $\begin{array}{l}3.15 \\
3.15 \\
\end{array}$ & $3 / 20$ & 3.7 & 9.4 \\
\hline TOTAL & & 3.7 & 6.3 & & & 6.3 & & 3.7 & 9.4 \\
\hline \multicolumn{10}{|l|}{ Wrong inversion } \\
\hline & $1 / 2$ & & 6.3 & $2 / 3$ & & 3.1 & $1 / 3$ & & 3.1 \\
\hline TOTAL & & & 6.3 & & & 3.1 & & & 3.1 \\
\hline
\end{tabular}




\begin{tabular}{|c|c|c|c|c|c|c|c|c|c|}
\hline \multicolumn{10}{|l|}{ Others } \\
\hline - computation & $25 / 15$ & 3.7 & & 3 & 3.7 & & $60 / 25$ & 3.7 & \\
\hline - number & $2.5 / 5$ & 3.7 & & & & & -3 & 3.7 & \\
\hline - hybrid & & & & $\begin{array}{c}1 / 2 \\
2 / 30\end{array}$ & 3.7 & 3.15 & & & \\
\hline - guessed & $.45 / .09$ & & 3.15 & $1.6 / .15$ & & 3.15 & $1.8 / 5$ & & 3.1 \\
\hline
\end{tabular}

